\title{
The role of tryptophan-rich basic protein (WRB) in inner hair cell synaptic transmission and hearing
}

\author{
Dissertation \\ for the award of the degree \\ "Doctor rerum naturalium" \\ Division of Mathematics and Natural Sciences \\ of the Georg-August University Göttingen \\ within the doctoral program "Sensory and Motor Neuroscience" \\ of the Georg-August-University School of Science (GAUSS)
}

submitted by

Iliana Panou

from

Athens, Hellas

Göttingen, 2013 
First Referee:

Second Referee:

Third Referee:

Date of oral examination: $\quad$ May $8^{\text {th }} 2013$
Dr. Tina Pangršič

Professor Dr. Nils Brose

Dr. Iwan A.T. Schaap 
Herewith I declare, that I prepared the PhD Thesis 'The role of tryptophan-rich basic protein (WRB) in inner hair cell synaptic transmission and hearing' on my own and with no other sources and aids than quoted.

Göttingen, April $2^{\text {nd }} 2013$. 



\section{Contents}

Table of Contents $\quad$ V

List of Figures $\quad$ ix

List of Abbreviations xi

$\begin{array}{ll}\text { Abstract } & \text { XV }\end{array}$

Acknowledgments $\quad$ xvii

1 Introduction 1

1.1 Mammalian Ear . . . . . . . . . . . . . . . . . . . 1

1.1.1 Basic Anatomy . . . . . . . . . . . . . . . . . . 1

1.1.2 Organ of Corti . . . . . . . . . . . . . 2

1.1.3 Innervation of the Cochlea . . . . . . . . . . . . . . . 3

1.1.4 From Sound Waves to Electrical Responses . . . . . . . . . . . 4

1.2 The IHC Ribbon Synapse . . . . . . . . . . . . . . . . . . . 6

1.2.1 Exocytic performance at the IHC ribbon synapse . . . . . . . 6

1.2.2 Molecular composition of the IHC ribbon . . . . . . . . . . . 7

1.2.3 Molecules involved in the IHC neurotransmission . . . . . . 8

1.3 Otoferlin, a multi- $\mathrm{C}_{2}$ domain protein . . . . . . . . . . . 9

1.3.1 Implication of otoferlin in deafness . . . . . . . . . . . . 10

1.3.2 Expression pattern of mouse otoferlin . . . . . . . . . . 11

1.3.3 Interaction partners of otoferlin . . . . . . . . . . . . 12

1.3.4 Evidence and hypotheses on otoferlin's function . . . . . . . . 12 
1.4 Tail-anchored Proteins . . . . . . . . . . . . . . . . . . 13

1.4.1 ER-targeting insertional pathways . . . . . . . . . . 14

1.4.2 Predictions on the profile of the membrane-target sequence . . 18

1.4.3 Tryptophan-rich Basic Protein - WRB . . . . . . . . . 18

1.5 RNA Interference - Mechanism and Applications . . . . . . . . . . . . 19

1.6 Aims of this study . . . . . . . . . . . . . . . . . . 21

2 Materials and Methods $\quad 23$

2.1 Materials . . . . . . . . . . . . . . . . . . . 23

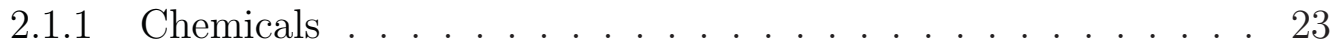

2.1.2 Enzymes, kits and bacterial strains . . . . . . . . . 23

2.1.3 miRNA sequences and oligomers . . . . . . . . . . . 23

2.1.4 Sequencing reactions and viral vector production . . . . . . 24

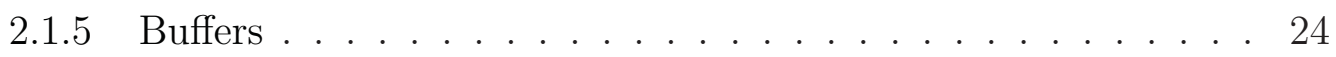

2.1.6 Antibodies........................ 26

2.1.7 Primers . . . . . . . . . . . . . . . . . 27

2.1.8 Animals ........................... 27

2.1 .9 Mouse lines . . . . . . . . . . . . . . . . . . 28

2.2 Methods . . . . . . . . . . . . . . . . . . . . 29

2.2.1 Cloning procedures . . . . . . . . . . . . . . 29

2.2.2 Cloning of the microRNA expressing vector . . . . . . . . 30

2.2.3 Cloning of the AAV1/2 -HBA-EGFP-miRNA vector . . . . . . 33

2.2.4 Ethanol precipitation of DNA . . . . . . . . . . 34

2.2.5 Bacterial transformation . . . . . . . . . . . . 34

2.2.6 Cell Culture . . . . . . . . . . . . . . . . 35

2.2.7 Cell Transfection Methods . . . . . . . . . . . . . . . . 35

2.2.8 Preparation of protein lysates . . . . . . . . . . . 37

2.2.9 Protein concentration estimation . . . . . . . . . . . 37

2.2.10 Western Blotting . . . . . . . . . . . . . 37

2.2.11 Viral transduction of HEK 293 cell line . . . . . . . . . . . 38

2.2.12 Embryonic transuterine otocyst injections . . . . . . . . . . . 39

2.2.13 Genotyping protocol of the pinball-wizard line . . . . . . . 39 
2.2.14 Whole-mount preparation of the organ of Corti . . . . . . . 40

2.2.15 RT-PCR . . . . . . . . . . . . . . . 40

2.2.16 Perforated patch-clamp recordings . . . . . . . . . . . . 41

2.2.17 Immunostaining . . . . . . . . . . . . . . . . . 42

2.2.18 Confocal microscopy and image analysis . . . . . . . . . . . 43

2.2.19 Electron Microscopy . . . . . . . . . . . . . . . . 43

2.2.20 Auditory Brainstem Responses . . . . . . . . . . . . 44

2.2.21 Distortion Product Otoacoustic Emissions (DPOAE) . . . . . 45

3 Results $\quad 46$

3.1 Characterization of the Pwi mouse phenotype . . . . . . . . . 46

3.1.1 The transgenic Pwi mouse line . . . . . . . . . . . . 46

3.1.2 The conditional Pwi knockout exhibits seizure-like events . . . 48

3.1.3 Deletion of $W r b$ causes a progressive hearing defect . . . . . 50

3.1.4 The morphology of the Pwi organ of Corti . . . . . . . . 53

3.1.5 The conditional Wrb construct is recombined only in the presence of Cre recombinase . . . . . . . . . . . 53

3.1.6 Disruption of synaptic function in $\mathrm{Pwi}^{\mathrm{flx} / \mathrm{flx}} \mathrm{VDCR}^{+} \mathrm{CGCT}^{+}$ and $\mathrm{Pwi}^{\mathrm{flx} / \mathrm{flx}} \mathrm{VDCR}^{-} \mathrm{CGCT}^{+} \mathrm{IHCs} \ldots \ldots . . . . . . .56$

3.1.7 Slowed vesicle pool replenishment in IHCs of $\mathrm{Pwi}^{\mathrm{fx} / \mathrm{fx}} \mathrm{VDCR}^{+}$ $\mathrm{CGCT}^{+}$mice . . . . . . . . . . . . . . . 58

3.2 Synaptic morphology . . . . . . . . . . . . . . . . . 60

3.2.1 Indication of a progressive reduction in the number of ribbonoccupied synapses of $\mathrm{Pwi}^{\mathrm{flx} / \mathrm{flx}} \mathrm{VDCR}^{+} \mathrm{CGCT}^{+} \mathrm{IHCs}$. . . . 60

3.2.2 Unusually large vesicles accumulate at the KO synapse . . . . 61

3.3 Effect of the $W r b$ deletion on the molecular composition of the IHC ribbon synapse . . . . . . . . . . . . . . . . . . . . . 65

3.3.1 Reduced otoferlin protein levels . . . . . . . . . . . . 65

3.3.2 Disruption of the protein WRB changes the distribution of the TRC40 interaction partner .............. 67

3.4 Silencing of otoferlin _ . . . . . . . . . . . . . . . . . . 69

3.4.1 In vitro silencing . . . . . . . . . . . . . . . . . . . . . 70 
3.4.2 In vivo silencing . . . . . . . . . . . . . . . . . . . . . . 73

3.4.3 Synaptic transmission of mouse otoferlin heterozygous animals 75

4 Discussion $\quad 78$

4.1 Seizures and lethality in the Pwi KO mice . . . . . . . . . . . . . . 79

4.2 Down-regulation of TRC40 in the absence of WRB . . . . . . . . . 80

4.3 Progressive hearing loss in Pwi KO mice is probably attributed to the dysfunction of $\mathrm{IHCs}^{2} \ldots \ldots$. . . . . . . . . . . . . . . . 81

4.3.1 Recombination in the Wrb gene . . . . . . . . . 82

4.3.2 A defect of the ultrastructure of IHC ribbon synapse . . . . . 83

4.4 Hypotheses on the function of WRB in IHCs . . . . . . . . . . . . . . 84

4.5 Silencing otoferlin in the mammalian inner ear . . . . . . . . . . 86

$\begin{array}{lr}\text { Bibliography } & 89\end{array}$ 


\section{List OF Figures}

1.1 Anatomy of the cochlea. . . . . . . . . . . . . . 3

1.2 The journey of a sound wave in the cochlea. . . . . . . . . . . . 5

1.3 Protein domain structure of otoferlin . . . . . . . . . . . . 11

1.4 The two main membrane insertional pathways. . . . . . . . . . . . . . 16

1.5 miRNA biogenesis pathway. . . . . . . . . . . . . . 20

2.1 The pEGFP-C1 backbone. . . . . . . . . . . . . . . . 31

3.1 Targeted disruption of the $W r b$ gene locus. . . . . . . . . . . . . 47

3.2 The behavior of the conditional Pwi KO mice. . . . . . . . . . . . . . 49

3.3 Assessment of hearing in Pwi mouse line. . . . . . . . . . . . . . . . . 51

3.4 Assessment of hearing-DPOAE. . . . . . . . . . . . . . 52

3.5 Cre activity is indicated by green fluorescent IHCs. . . . . . . . . . . 54

3.6 Testing the recombination by RT-PCR. . . . . . . . . . . . . 55

3.7 IHC synaptic function of the Pwi mouse line. . . . . . . . . . . . 57

3.8 Synaptic vesicle replenishment of $\mathrm{Pwi}^{\mathrm{flx} / \mathrm{flx}} \mathrm{VDCR}^{+} \mathrm{CGCT}^{+} \mathrm{IHCs}$ is slowed down. . . . . . . . . . . . . . . . . . . . 59

3.9 Quantification of ribbon synapses. . . . . . . . . . . . . . . . 62

3.10 Ultrastructural analysis of the Pwi ribbon synapse. . . . . . . . . . . 63

3.11 Ultrastructural analysis of the Pwi ribbon synapse (controls). . . . . 64

3.12 Quantification of ultrastructural analysis of the IHC ribbon synapses. 65

3.13 Expression pattern of otoferlin distribution in the first cochlear turn. $\quad 66$

3.14 Expression pattern of otoferlin distribution in apical cochlear IHCs. . 67

3.15 Semi-quantitative evaluation of otoferlin protein levels. . . . . . . 68 
3.16 Reduced expression of TRC40 . . . . . . . . . . . . . . . . 69

3.17 Construction of the silencing vectors. . . . . . . . . . . . . 70

3.18 In vitro silencing - Protein level quantification. . . . . . . . . . . . . 71

3.19 In vitro silencing. . . . . . . . . . . . . . . . . . . . . . . . . 73

3.20 Silencing of otoferlin in mouse IHC - First viral preparation. . . . . . 74

3.21 Silencing of otoferlin in mouse IHC - Second viral preparation

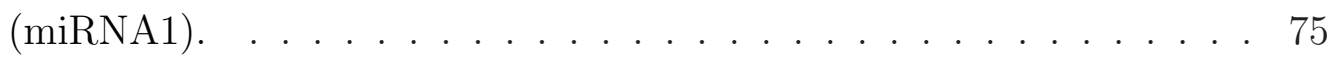

3.22 Silencing of otoferlin in mouse IHCs - Second viral preparation (scramble1). . . . . . . . . . . . . . . 76

3.23 Exocytic performance of Otof $^{+/}$IHCs. . . . . . . . . . . . 77 


\section{List of ABBRIEVIATions}

IHC: Inner Hair Cell

OHC: $\quad$ Outer Hair Cell

ANF: Auditory Nerve Fiber

SGN: Spiral Ganglion Neuron

MET: Mechanoelectrical Transduction

AZ: Active Zone

CNS: Central Nervous System

SV: Synaptic Vesicle

AMPA: $\quad \alpha$-amino-3-hydroxy-5-methylisoxazole-4-propionic acid

RRP: Readily Releasable Pool

SNARE: N-ethylmaleimide-sensitive factor attachment protein receptor

TMD: Transmembrane Domain

TA: Tail-Anchored

ER: Endoplasmic Reticulum

RISC: Ribonucleoprotein complex

SRP: Signal Recognition Particle

RNAi: RNA interference

PBS: Phosphate Buffer Saline

EDTA: Ethylenediaminetetraacetic acid

NP-40: Octyl phenoxypolyethoxyethanol

SDS: Sodium Dodecyl Sulfate

PMSF: Phenylmethanesulfonylfluoride

DTT: Dithiothreitol 
HEPES: 4-(2-hydroxyethyl)-1-piperazineethanesulfonic acid

Tris-HCl: $\quad$ Tris hydrochloride

$\mathrm{NaCl}$ : Sodium chloride

$\mathrm{Na}_{2} \mathrm{HPO}_{4}$ : Disodium phosphate

$\mathrm{KCl}$ : Potassium chloride

CsCl: Cesium chloride

$\mathrm{CaCl}_{2}$ : Calcium chloride

$\mathbf{M g C l}_{2} \cdot \mathbf{6} \mathrm{H}_{2} \mathrm{O}$ : Magnesium chloride hexahydrate

$\mathrm{MgSO}_{4} \cdot \mathbf{7} \mathbf{H}_{2} \mathrm{O}$ : Magnesium sulfate heptahydrate

$\mathrm{NaHCO}_{3}$ : Sodium bicarbonate

NaOAc: Sodium Acetate

HEK: Human Embryonic Kidney

FBS: Fetal Bovine Serum

FCS: Fetal Calf Serum

BCA: Bicinchoninic acid

TEMED: $\quad$ N, N, N', N'-tetramethylethylenendiamine

FA: Formaldehyde

PNK enzyme: Polynucleotide kinase

Cs-gluconate: Cesium gluconate

TEA-Cl: Tetraethylammonium Chloride

4-AP: 4-aminopyridine

DMSO: Dimethylsulfoxide

EGFP: Enhanced Green Fluorescent Protein

DAPI: 4', 6-Diamidino-2-Phenylindole

ITRs: Inverted Terminal Repeats

AAV: Adeno-associated Virus

CMV: Cytomegalovirus

HBA: Human b-actin

WPRE: Woodchuck Post-transcriptional Regulatory Element

BGH: Bovine Growth Hormone

ABR: Auditory Brainstem Response 
SPL: $\quad$ Sound Pressure Level

DPOAE: Distortion Product Otoacoustic Emission 


\begin{abstract}
The tryptophan-rich basic protein (WRB) has recently been proposed to act as the receptor of tail-anchored (TA) proteins. Together with the cytosolic protein TRC40 it presumably builds the main components of the posttranslational membrane insertion pathway in mammalian cells. A mutation in the wrb homologue gene in zebrafish causes visual, hearing, and balance deficits, as observed in the pinball-wizard $(p w i)$ zebrafish mutants. In this study, I investigated the role of WRB in hearing by studying the synaptic transmission at the first auditory synapse, the cochlear inner hair cell (IHC) ribbon synapse in the Pwi conditional knockout mice. In this mouse model, the Wrb gene was targeted for excision through Cremediated homologous recombination in tissues that express the vesicular glutamate transporter-3. The findings indicated a progressive hearing loss accompanied by epileptic attacks and vestibular deficits. Further, the knockout animals did not survive beyond two months after birth. Patch-clamp recordings revealed a normal exocytosis of the readily-releasable pool of vesicles in IHCs, but diminished sustained component of exocytosis, suggesting a defect in vesicle replenishment. These findings were further supported by ultrastuctural analysis of the knockout IHC ribbon synapses, where unusually large vesicle clusters were observed together with significantly fewer ribbon-associated vesicles. Otoferlin, a putative calcium sensor for synaptic vesicle fusion and/or priming factor at the IHC ribbon synapse, has recently been predicted to be a TA protein. In support of the hypothesis that the disrupted pathway of membrane insertion could affect the molecular components of the IHC synapse, otoferlin protein levels in IHCs of the Pwi knockout mice were decreased. Moreover, the expression of TRC40 was also strongly reduced. In conclusion, the IHC synaptic dysfunction probably contributes to the progressive hearing loss in the Pwi knockout mice. Although not yet quite understood, this synaptic defect might be attributed to deficits in certain IHC synaptic proteins, like otofelin that are disrupted upon deletion of the WRB protein.

Furthermore, as a parallel study, a viral silencing approach was established, utilizing adeno-associated viruses for the delivery of miRNA-like silencing molecules in the developing otocyst. The results of the effort to knock-down the protein levels of
\end{abstract}


otoferlin were suggestive of the success of the method, which nevertheless, needs further optimization. 


\section{Acknowledgments}

First and foremost, I would like to express my deepest appreciation and gratitude to Professor Dr. Tobias Moser for accepting me in his scientific team, providing to me a scientific "shelter" with numerous opportunities and encourage my efforts. Special thanks to Professor Dr. Nils Brose and Dr. Iwan A.T. Schaap for being members of my $\mathrm{PhD}$ committee and for advising me on my work.

I would like to thank Dr. Tina Pangršič for introducing me into the exciting "world" of electrophysiology, teaching me the experiments and provide me with help for their analysis and understanding.

I want to thank all my colleagues at the Inner Ear Lab for the pleasant and relaxed atmosphere that enabled fruitful interactions and creative work. Special thanks to Dr. Jakob Neef and Gerhard Hoch for their generous and constant help, to Christiane Senger-Freitag and Sandra Gerke for excellent technical support, to Nadine Hermann for her professional attitude and personal friendship, and Frau Brigitte Higazi for her amazing "courage" and positiveness to deal with every single practical detail.

My special thanks to my close friends and colleagues, Nikolai Chapochnikov, Tzu-Lun Wang, and Linda Hsu for their continuous support, truthful interaction and full of fan moments, inside and outside the lab.

I am grateful to Dr. SangYong Jung, whom his scientific input and guidance had an invaluable contribution to my work and personal thinking.

Last, but not least, I owe to thank my family

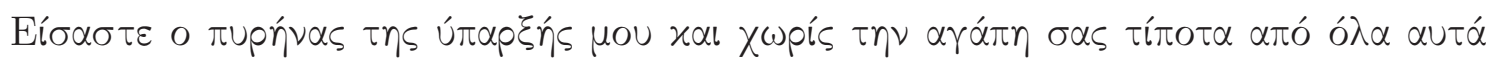

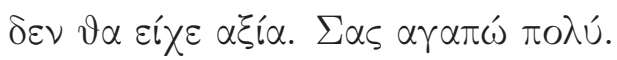




\section{1}

\section{INTRODUCTION}

Animals have evolved over millions of years in continuously changing environments, expanding and dominating a variety of new milieus. Their chances of survival and successful settlement in a habitat are defined by the senses that they have developed. The sense of hearing appeared quite early, originating in the Triassic period (250-220 million years ago), when the first primitive hearing organ, a short auditory papilla of few hundred hair cells, was established and developed thereafter simultaneously in the three major amniote vertebrate lineages, i.e., mammals, birds, and reptiles (Manley, 2000). In invertebrates, hearing, is mostly related to gravity and vibration changes, captured by ciliated primary sensory cells located in different regions of the body. Hearing and the auditory system, respectively, enabled animals to capture sound and to distinguish differences in its temporal characteristics, amplitude, and spatial profile and thus, to respond effectively to the demands of their environment. For humans is an invaluable gift, enriching our social interaction and enhancing our comprehension of the world.

\subsection{Mammalian Ear}

\subsubsection{Basic Anatomy}

The work of great scientists, such as the physiologist Hermann von Helmholtz (18211894) and the anatomist Marquis Alfonso Corti (1822-1876), on preservation techniques for the mammalian auditory system, contributed grandly to the anatomical characterization of the auditory system and the understanding of the physical properties of sound transduction, from a traveling wave along the cochlea to an electrical response by the sensory cells. 
The hearing organ in mammals is divided into three anatomical parts: the external ear with the ear canal, the middle ear with the ear drum and ossicle chain, and the inner ear with the cochlea and vestibular organs. The middle and inner ear are housed inside the temporal bone. The inner ear is well protected inside the bony labyrinth. The cochlea (from the Greek word $\kappa o \chi \lambda \iota \alpha \varsigma$, meaning snail) consists of a system of fluid-filled cavities that are separated from each other by Reissner's membrane and the basilar membrane. Two of these cavities, i.e., the scala vestibuli and scala tympani (Figure $1.1 \mathrm{~b}$ ) are filled with perilymph (a high-sodium solution similar to the extracellular solution elsewhere in the body) and are linked at the apex of the cochlea at the helicotrema. Between them is the scala media, which houses the auditory sensory epithelium, i.e., the organ of Corti. The organ of Corti primarily consists of sensory cells and supporting cells and lies on the basilar membrane (Figure $1.1 \mathrm{c}$ ). The apical side of the sensory epithelium is bathed in endolymph (high-potassium solution). The lateral wall of scala media is made up of the stria vascularis, which is rich in blood vessels and establishes the ionic composition and electrical potential of the endolymph.

\subsubsection{Organ of Corti}

The organ of Corti is an epithelium of $\sim 11 \mathrm{~mm}$ length in mice and $\sim 34 \mathrm{~mm}$ in humans (Ashmore, 2008). Two types of sensory receptor cells are positioned in 4 distinct rows throughout the entire length of the organ of Corti, i.e., one row of inner hair cells (IHCs) and 3 rows of outer hair cells (OHCs). Different types of supporting cells and nerve fibers surround these cells. The sensory hair cells have two distinct surfaces, i.e., apical and basolateral, which differ in structure and function. The apical side receives the auditory stimulus, while the basolateral surface transmits the signal through the synapses that are formed there. On their apical surface, these cells display microvilli, composed of parallel actin filaments, the "stereocilia", which form the hair bundle. Stereocilia are actively motile structures, connected with each other via tip links and lateral links, which hold the bundle together. The bundle is subjected to a fine-coordinated movement every time an appropriate auditory signal arrives in the cochlea. The bundles of the OHCs are attached to the 


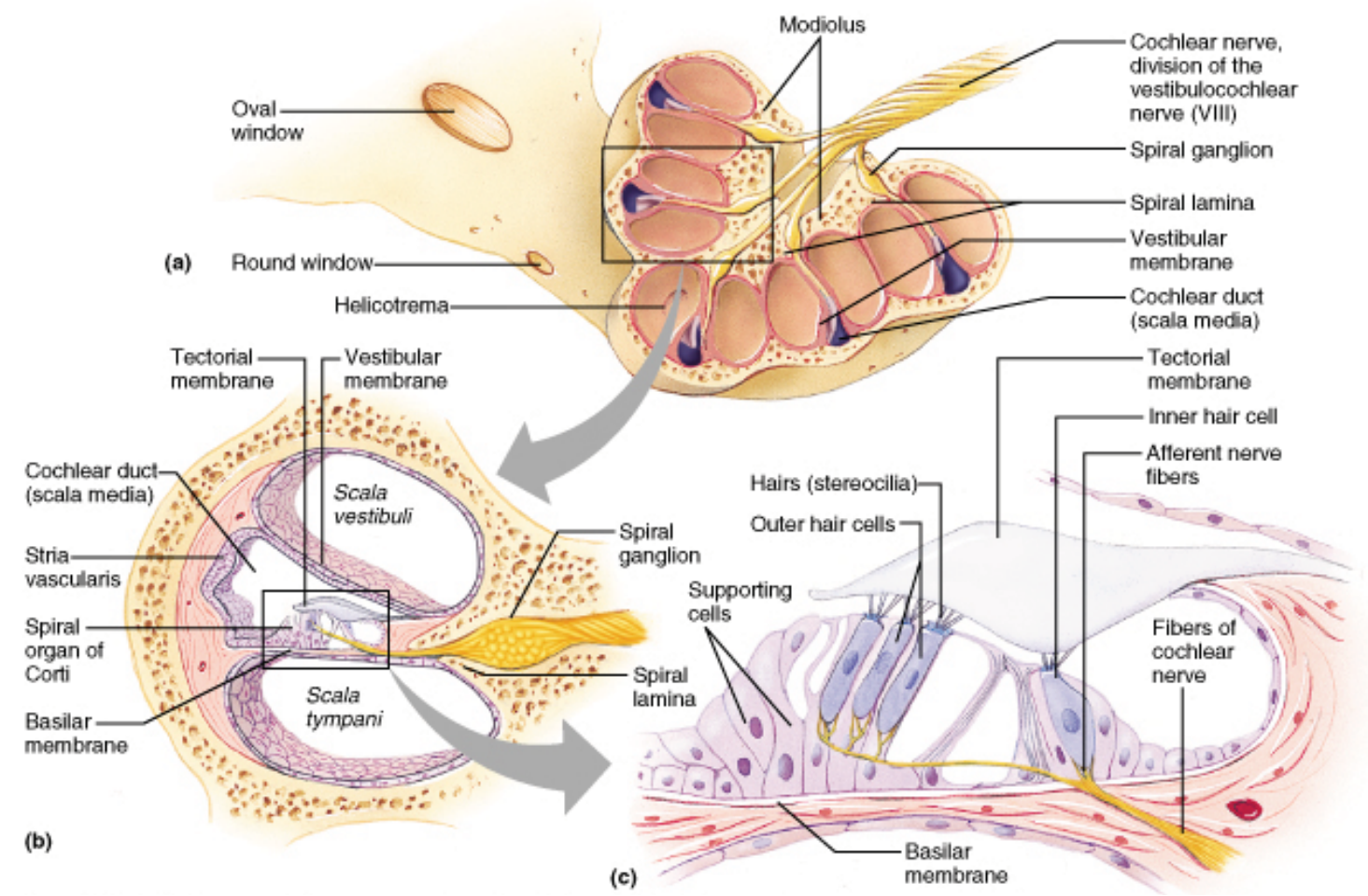

Figure 1.1: Anatomy of the cochlea. (a) Anatomical drawing of a cross-section of the whole cochlea depicting the eighth cranial nerve as it exits the cochlea and the cochlear ducts, (b) one cross-section of the cochlea, indicating the fluid-filled cavities, scala vestibuli, scala media, and scala tympani and the position of the organ of Corti, (c) the organ of Corti with the two types of sensory cells (IHCs and OHCs) and the supporting cells. (Figure adapted from Pearson Education Inc., 2006)

tectorial membrane and are exposed to movements whereas the bundles of the IHCs are bathed inside the endolymph and hence, are subjected to deflections caused by the fluid movements.

\subsubsection{Innervation of the Cochlea}

The central axis of the cochlea is the bony modiolus, which shapes a passageway for the afferent (toward the brain) and efferent (toward the periphery) auditory nerve fibers (ANFs). It houses the somata of the bipolar spiral ganglion neurons (SGN) that give rise to afferent ANFs and send peripheral neurites contacting the IHCs and OHCs, respectively. In mice, each IHC forms synapses with $\sim 20$ unbranched 
neurites of type I SGN (Meyer and Moser, 2010). ANFs of type I SGN are thick and myelinated fibers. In cats, it has been demonstrated that the afferent nerve fibers innervating the pillar side of IHCs have a large diameter, low threshold, and high spontaneous firing rate, while those from the modiolar side have a small diameter, high threshold, and low spontaneous firing rate (Merchan-Perez and Liberman, 1996). Additionally, lateral olivocochlear neurons (efferents) project to the afferent fibers beneath IHCs. However, early in development, in mice, the efferents terminate directly on the IHCs (Sobkowicz and Slapnick, 1994). Conversely, the afferent innervation of the OHCs consists of a small number of thin, unmyelinated fibers originating from type II SGNs. One such afferent may contact 6-100 OHCs, most often within the same row (Peter Dallos et al., 1996). Most of the innervation of the OHCs comes from large-diameter efferent myelinated fibers, i.e., the medial olivocochlear neurons. All the afferent ANFs exit the cochlea and project centrally to the brainstem via the eighth cranial nerve, up to the auditory cortex. Every individual ANF responds to a slightly different characteristic frequency that is determined by the position of the fiber termination in the organ of Corti (Dallos, 1992; Liberman, 1982). This implies that every hair cell is capable of recognizing and responding to a specific range of sound frequencies as a consequence of active and passive cochlear micromechanics. This tonotopic organization of the entire cochlea, whereby high frequency and low frequency sound cause vibrations in the base and the apex of the cochlea, respectively, is followed by tonotopicity throughout the afferent auditory pathway up to the auditory cortex.

\subsubsection{From Sound Waves to Electrical Responses}

Sound waves are collected by the external ear and travel through the ear canal reaching the tympanic membrane. There, they induce mechanical vibrations of the tympanum and subsequently set the ossicular chain (malleus, incus, stapes), which is connected to the tympanic membrane, into motion. The movement of the stapes causes a traveling wave of the basilar membrane. As a consequence, the organ of Corti is leading to mechanoelectrical transduction by inner and outer hair cells. Deflection of the hair bundles of inner and outer hair cells is a well organized 
process, which modulates tension on the tip links, directing the open probability of cation-selective mechanoelectrical transduction (MET) channels.

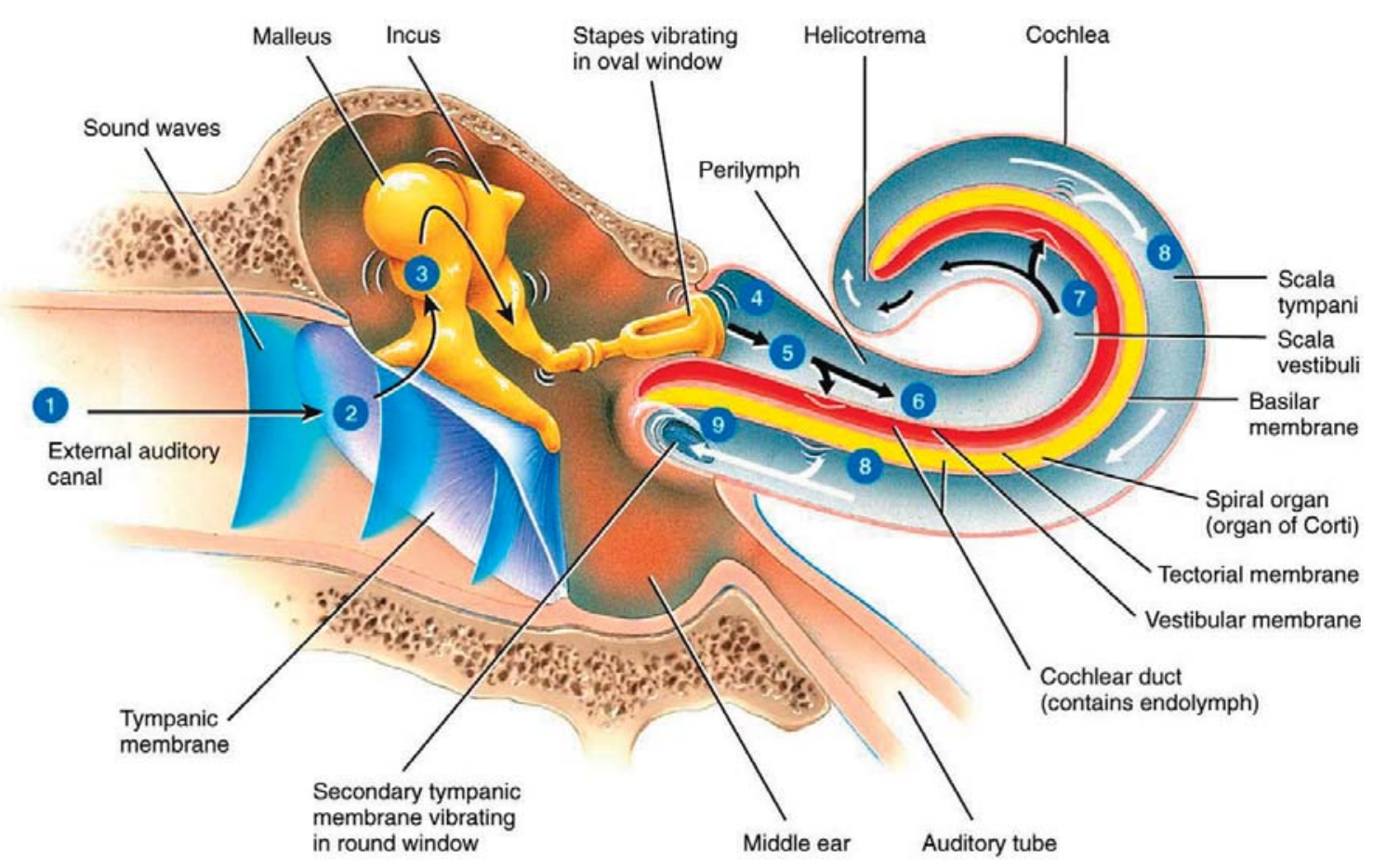

Figure 1.2: The journey of a sound wave in the cochlea. Illustration which shows the middle and inner ear with uncoiled cochlea for simplicity. The sound waves reach the tympanic membrane and set the ossicular chain (malleus, incus, and stapes) into motion. The forces of this motion create pressure in the entire cochlear ducts by setting perilymph in circulation. The distance that a traveling wave spans inside the cochlea, depends on its frequency. (Figure adapted from John Wiley and Sons, Inc.)

Displacement of the hair bundle toward the longest stereocilium (positive displacement) increases the open probability of MET channels and results in the influx of mostly $\mathrm{K}^{+}$ions. The ensuing receptor potential drives prestin-mediated electromotility in OHCs (Dallos, 2008; Zheng et al., 2000) and mediates the influx of $\mathrm{Ca}^{2+}$ from voltage-sensitive calcium channels and facilitates the release of neurotransmitter from the afferent synapses in IHCs and OHCs. 


\subsection{The IHC Ribbon Synapse}

The auditory sensory system is unique in the nervous system in its accuracy and speed of the transduction of stimuli, encoding, and representation in the primary auditory cortex. IHCs are the genuine sensory cells of the auditory system, which sustain neurotransmission continuously, in response to graded changes of membrane potential. To accomplish these roles, IHCs form specialized synapses, equipped with a sophisticated protein machinery.

Chemical synapses enable the communication between two sensory/nerve cells by synaptic vesicle exocytosis, which is restricted to a small section of the presynaptic plasma membrane, the active zone (AZ). The active (AZ) of every synapse should fulfill three principal functions: i) collect a sufficient number of vesicles to be available for release upon stimulus, ii) recruit voltage-gated calcium channels, where $\mathrm{Ca}^{2+}$ enters and triggers exocytosis, iii) couple the presynaptic site with the postsynaptic neuron with cell-adhesion molecules. The proteins that localize at the AZ create a cytomatrix (Zhai and Bellen, 2004) and assist the structural organization of the synapse, as well as the vesicle release (exocytosis) and retrieval (endocytosis).

IHC synapses differ from synapses of the central nervous system (CNS) in many aspects. They are equipped with an electron-dense structure called the synaptic ribbon, which is anchored to the active zone and extends from the presynaptic site into the cytoplasm. In the mouse IHCs ribbons vary in shape and size (100-400 $\mathrm{nm}$ ), typically appear to be $\leq 200 \mathrm{~nm}$ in width (Moser et al., 2006). Depending on the position of the IHC along the tonotopic axis of the cochlea, the number of ribbon synapses varies (Meyer et al., 2009). The functional relationship between a ribbon-type AZ in the presynaptic IHC and an afferent postsynaptic neuron comprises the afferent synapse, which carries the "responsibility" of maintaining an "inexhaustible" supply of synaptic vesicles (SV).

\subsubsection{Exocytic performance at the IHC ribbon synapse}

The active zone, with its hallmark the ribbon, serves as a platform for the fusion of vesicles, carrying the neurotransmitter glutamate upon $\mathrm{Ca}^{2+}$ influx from $\mathrm{Ca}_{v} 1.3$ 
L-type voltage-gated calcium channels (Brandt et al., 2003; Platzer et al., 2000). Glutamate is loaded into the vesicles by the glutamate transporter VGLUT3, the exclusive glutamate transporter isoform in IHCs. Lack of VGLUT3 leads to deafness (Ruel et al., 2008; Seal et al., 2008). When glutamate is released into the synaptic cleft it binds to postsynaptic $\alpha$-amino-3-hydroxy-5-methylisoxazole-4-propionic acid (AMPA) receptors and triggers the generation of action potentials in afferent neurons (Glowatzki and Fuchs, 2002; Rutherford et al., 2012).

The main contribution of the ribbon to the synaptic transmission is the accumulation of synaptic vesicles close to the AZ. Indeed, different populations of SVs have been identified around the ribbon; either associated with it, or in close proximity, or beneath the ribbon, docked to the presynaptic membrane (Lenzi et al., 1999). Therefore, multiple roles have been attributed to the ribbon, i.e., it tethers vesicles, which are thought to be involved in exocytosis (LoGiudice et al., 2008; Snellman et al., 2011), it may stabilize the multiple release sites within the AZ (Frank et al., 2010; Khimich et al., 2005), and/or may promote homotypic fusion of synaptic vesicles before exocytosis (Parsons and Sterling, 2003).

In mouse IHCs, ribbon tethers $~ 100-200$ vesicles (Khimich et al., 2005). Upon stimulus application, the mature IHCs respond in two subsequent phases, i.e., a phase of fast and slow vesicle release. The fast phase is attributed to the fusion of vesicles that are docked, close to the calcium channels. They sense the $\mathrm{Ca}^{2+}$ influx and undergo exocytosis (readily releasable pool of vesicles [RRP]; RRP size: $7.4 \mathrm{fF}, 200$ vesicles) (Beutner and Moser, 2001). The slow phase is believed to primarily represent the replenishment of the vesicles into the RRP (Meyer et al., 2009; Nouvian et al., 2006) Pangršič et al., 2010) and their subsequent fusion (Moser and Beutner, 2000).

\subsubsection{Molecular composition of the IHC ribbon}

The main structural component of the ribbon, and also unique to this structure, is RIBEYE. It consists of the unique aminoterminal, proline-rich-A domain, and a B domain, identical to the C-terminal domain of the nuclear co-repressor protein 2 (CtBP2). RIBEYE domains mediate homotypic interactions that support the role 
of RIBEYE as a scaffold molecule (Schmitz et al., 2000). CtBP2 and its isoform CtBP1, localize both at the ribbon (tom Dieck et al., 2005).

Known interaction partner of RIBEYE/CtBP2 is bassoon (tom Dieck et al., 2005), which together with piccolo consist the cytomatrix scaffold of the ribbon. Bassoon's role at ribbon synapses has been suggested to be the anchoring of the ribbon to the active zone, after the finding that in a bassoon partial-deletion mouse mutant, ribbons were absent or mislocalized from the AZ (Khimich et al., 2005). Inner hair cells of these mice have a smaller RRP and reduced sustained exocytosis (Frank et al., 2010; Khimich et al., 2005), as well as perturbed clustering and organization of $\mathrm{Ca}_{v}$ 1.3 L-type voltage-gated calcium channels at the AZ (Frank et al., 2010). Thus, it appears that bassoon together with the ribbon organize the calcium channels at the presynaptic sites. The role of piccolo in hair cells remains to be clarified.

ERC (ELKS/CAST) and Rab3-interacting proteins (RIMs) have been identified as further scaffold molecules of the ribbon-type AZ of photoreceptors (tom Dieck et al., 2005; Ohara-Imaizumi et al., 2005), whose presence at the IHC synapse still need to be verified (Gebhart et al., 2010). Interactions with the channels are also promoted by RIMs and RIM-binding proteins, with the first binding through their PDZ domains to the $\alpha$ subunits of L-type calcium channels (Coppola et al., 2001). In CNS synapses RIMs participate at vesicle docking and priming (Deng et al., 2011).

\subsubsection{Molecules involved in the IHC neurotransmission}

The fusion of synaptic vesicles with the presynaptic membrane is accomplished by interactions of plasma membrane-associated and vesicle-associated proteins. Being first reported in CNS synapses, the N-ethylmaleimide-sensitive factor attachment protein receptors (SNAREs), which include the vesicular proteins synaptobrevin 1 and 2 and the presynaptic membrane proteins syntaxin 1 and SNAP-25, consist the minimal protein machinery for fusion between two membranes. Complexins, synapsins, and synaptophysins participate also in this process. Finally, calcium, which is the triggering signal for fusion to happen, binds to the calcium sensor synaptotagmin and this initiates the protein-protein interactions that will lead the SVs to fusion. 
The scenery at the IHC synapse is different. As opposed to CNS, synaptophysins, synapsins (Safieddine and Wenthold, 1999), and complexins (Strenzke et al., 2009) have not been detected and no exocytosis defect was even observed in a complexin knockout (KO) mouse (Strenzke et al., 2009). Controversial evidence exists about the expression of neuronal SNAREs in IHCs. Whereas their expression was witnessed by many individual efforts (Safieddine and Wenthold, 1999; Uthaiah and Hudspeth, 2010), some more recent evidence argued against the presence of neuronal SNARE proteins in IHCs (Nouvian et al., 2011). Additional efforts to cleave SNAREs by clostridial neurotoxins did not block exocytosis (Nouvian et al., 2011). Still, their expression is further debated and the question of how IHCs mediate synaptic vesicle fusion remains open.

Furthermore, the expression and function of the vesicle proteins synaptotagmins at IHCs have not yet been fully clarified. Synaptotagmins are a big family (15 isoforms) of $\mathrm{C}_{2}$ domain proteins, many of which bind $\mathrm{Ca}^{2+}$ and phospholipids. Synaptotagmins 1 and 2 are considered to serve as the main calcium senors of SV fusion in CNS synapses and interact with SNAREs. Nevertheless, at mature IHCs synapses, neither synaptotagmin 1 nor synaptotagmin 2 are expressed (Beurg et al., 2010; Reisinger et al., 2011). Alternatives for the calcium sensor at IHCs have been proposed to be synaptotagmin 4 and otoferlin. The first one has been detected in IHCs (Safieddine and Wenthold, 1999) and was suggested to be required for linearizing the $\mathrm{Ca}^{2+}$ dependence of exocytosis (Johnson et al., 2010). Otoferlin is so far the most prominent candidate for calcium sensor (Roux et al., 2006) (see later), but still, the precise nature of its function is not yet deciphered. To conclude so far, IHC ribbon synapses seem to operate with an unconventional exocytic machinery differing from that of CNS synapses.

\subsection{Otoferlin, a multi- $\mathrm{C}_{2}$ domain protein}

Otoferlin is a member of the ferlin family, an ancient protein family existing throughout the eukaryotic kingdoms (Lek et al., 2012). The main characteristic domains of ferlins are the FerB domain of unknown function and the multiple cytosolic $\mathrm{C}_{2}$ domains. Ferlins contain between $5-7$, with otoferlin probably containing $6\left(\mathrm{C}_{2} \mathrm{~A}-\right.$ 
F). Synaptotagmins, known as regulators of calcium-mediated membrane fusion, have two such domains, both binding calcium and phospholipids. X-ray analysis of the crystal structure of the otoferlin $\mathrm{C}_{2} \mathrm{~A}$ domain revealed an eight $\beta$-strand structure, typical for $\mathrm{C}_{2}$ domains, which was folded with type II topology (Helfmann et al., 2011). In contrast to $\mathrm{C}_{2} \mathrm{~A}$ domain, the other $\mathrm{C}_{2}$ domains of otoferlin can bind calcium and phospholipids (Johnson and Chapman, 2010), although this is controversial for $\mathrm{C}_{2} \mathrm{~F}$ (Pangršič et al., 2010). Otoferlin displays a single C-terminal transmembrane domain (TMD). The existence of the TMD close enough to the C-terminus necessitates posttranslational insertion into the target membrane, as is typical for tail-anchored proteins (TA) (Lek et al., 2012).

Otoferlin protein shares $28 \%$ homology with the Caenorhabditis elegans FER-1 protein (Achanzar and Ward, 1997) and 38\% homology with the human dysferlin (Yasunaga et al., 1999). Reports from various pathologies caused by mutations in ferlin proteins, have indicated that the members of this family participate in cellular trafficking (Bernatchez et al., 2009; Doherty et al., 2005), membrane repair (Covian-Nares et al., 2010), and possibly calcium-triggered processes (Bansal et al., 2003; Dulon et al., 2009). Characteristic examples of pathologies caused by ferlin proteins are the muscular dystrophy, caused by an autosomal recessive inherited mutation in dysferlin and non-syndromic deafness, attributed to a mutation in otoferlin (Yasunaga et al., 1999).

\subsubsection{Implication of otoferlin in deafness}

The identification of a point mutation inside exon 18 of human otoferlin gene $(O T O F)$ was the first time ever that otoferlin protein was linked with hearing. The mutation, which produced a truncated protein form, was inherited in an autosomal recessive way and caused prelingual, non-syndromic hearing loss (DFNB9) (Yasunaga et al., 1999). To date, more than 60 pathogenic mutations have been identified in the OTOF gene (Rodrguez-Ballesteros et al., 2008) (Figure 1.3), which include nonsense and missense mutations that often affect the $\mathrm{C}_{2}$ domains. Pathogenic otoferlin mutations cause auditory synaptopathies, where hearing is impaired, but the otoacoustic emissions are mostly intact, suggesting that otoferlin is not impli- 
cated in the transduction mechanism of OHCs.

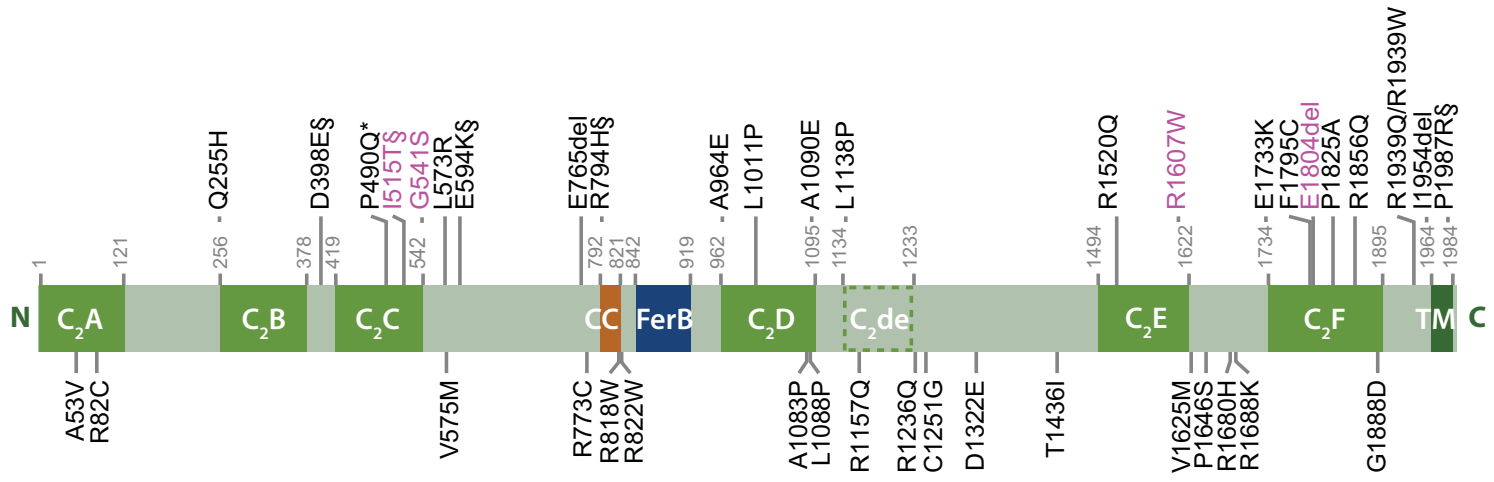

Figure 1.3: Protein domain structure of otoferlin. Illustration showing the structure of otoferlin, with $6 \mathrm{C}_{2}$ domains and one C-terminal transmembrane domain. Known pathogenic mutations of otoferlin are presented above the structure, and sequence variants on the bottom. (Figure adapted from Pangršič et al., 2010).

\subsubsection{Expression pattern of mouse otoferlin}

The expression profile of otoferlin in mouse cochlea changes during development. By means of immunostaining, it has been shown (Roux et al., 2006) that expression appears first at embryonic day 16 (E16) in IHCs and at E18 in OHCs. Gradually becomes more abundant in both cell types, reaching a maximum of fluorescent signal at P6. While in IHCs otoferlin persists during development and mature life, in OHCs, vanishes after P6. In the brain, it was detected in cerebellum and hippocampus (Roux et al., 2006). An additional study, proved the same expression in cochlear IHCs, suggesting a further detection until P9 at the basal part of OHCs (Schug et al., 2006). Furthermore, with in situ hybridization and RT-PCR, the same study showed the expression of otoferlin in different areas of the brain (brainstem, cerebellum, cortex, inferior colliculus, superior colliculus, hippocampus). The distribution pattern of otoferlin in IHCs appears throughout the cytoplasm (Roux et al., 2006; Schug et al., 2006). Complementary evidence from immunostaining of IHCs indicates a substantial amount of otoferlin in the presynaptic membrane distribution (Pangršič et al., 2010). 


\subsubsection{Interaction partners of otoferlin}

Otoferlin was shown to interact with the heterodimer syntaxin 1/SNAP-25 (Johnson and Chapman, 2010; Ramakrishnan et al., 2009; Roux et al., 2006) and also individual $\mathrm{C}_{2}$ domains or combinations of them could support SNARE-mediated liposome fusion in a $\mathrm{Ca}^{2+}$ dependent manner (Johnson and Chapman, 2010). However, as mentioned earlier, the expression of neuronal SNAREs at the IHC synapse is questionable (Nouvian et al., 2011) and therefore these interactions may not be relevant in vivo.

Other suggested interactions of otoferlin are with the Rab8b GTPase, at the apical region of the IHCs (Heidrych et al., 2008), with Myosin VI (Heidrych et al., 2009) and with Ergic2 (Zak et al., 2012). Rab8b has been shown to be involved in sorting and/or storage of neural recycling endosomes (Gerges et al., 2004), which suggests participation of otoferlin in endocytosis and the transport of vesicles to the active zone. However, this hypothesis requires more experimental evidence.

\subsubsection{Evidence and hypotheses on otoferlin's function}

Comparison of otoferlin with synaptotagmin has guided the research on the exact role for otoferlin. Insight into the role of otoferlin came initially from the study of an otoferlin KO mouse model (Roux et al., 2006). Deletion of the protein caused profound deafness and abolition of $\mathrm{Ca}^{2+}$-triggered exocytosis (P6 and P15), while the number of ribbon-associated and docked synaptic vesicles remained normal (at P15). In addition, the number of synapses in IHCs was reduced by $\sim 50 \%$ in P15 animals. These data were the first evidence for implicating otoferlin into a late step of exocytosis (e.g., priming or fusion) (Roux et al., 2006). Another mouse model, which enhanced the understanding of the role of otoferlin, was generated with a missense mutation (Asp-to-Gly) inside the $\mathrm{C}_{2} \mathrm{~F}$ domain (Schwander et al., 2007). This mutation, called pachanga, caused a reduction by $70 \%$ to otoferlin protein levels in IHCs and deafness, as indicated by the lack of auditory brainstem responses (ABR). In response to depolarization, IHCs of this mutant displayed normal RRP exocytosis, but impaired sustained response, indicative of inability to mantain efficient vesicle replenishment (Pangršič et al., 2010). The authors specifically 
suggested that otoferlin might be involved in priming as the synapse ultrastructure seemed unaltered. Moreover, this point mutation caused a slight change in the secondary structure of otoferlin, as indicated by circular dichroism spectroscopy. Thus, it was proposed that the reduced protein levels and/or the structural change on otoferlin may impair its function and the interactions established with other proteins (Pangršič et al., 2010).

Support of the hypothesis that otoferlin is the calcium sensor at IHC ribbon synapses, is enhanced by the absence of synaptotagmins 1 and 2 from these synapses (Beurg et al., 2010; Reisinger et al., 2011). The phenotype of the otoferlin KO mouse could however not be rescued by overexpressing synaptotagmin 1 in IHCs, which might happen due to a lack of appropriate interaction partners of synaptotagmins at the IHC synapse (Reisinger et al., 2011).

Gathering the facts about otoferlin, we could conclude that it is a membrane protein, localized throughout the IHC cytoplasm and the presynaptic membrane, with the ability to bind calcium and phospholipids. It is most probably implicated into a late step of exocytosis, promoting priming of the synaptic vesicles (Pangršič et al., 2010), and maybe enabling vesicle replenishment. Beyond the role as calcium sensor, it is speculated that otoferlin participates in the intracellular trafficking of vesicles from the Golgi network to endosomes and later to the basolateral membrane of IHCs (Schug et al., 2006; Yasunaga et al., 1999). If this is true, then the subcellular distribution can be better explained. It would suggest that before otoferlin reaches the synaptic site, it should get loaded into the vesicle membrane. How and where this process happens and which other proteins assist otoferlin is not yet known. Being a tail-anchored (TA) protein, predicted by a bioinformatics screen (Kalbfleisch et al., 2007), otoferlin must follow a specific pathway until it acquires the final topology on the membrane.

\subsection{Tail-anchored Proteins}

The plethora of integral membrane proteins utilize diverse mechanisms for insertion into phospholipid bilayers. Most of these proteins need to be translocated to the 
rough endoplasmic reticulum (ER) to acquire their proper folding and posttranslational modifications before they are sorted to their ultimate cellular compartments. This translocation can happen either during translation of the nascent polypeptide chain or after termination of translation, depending on the position of the membranetargeting signal.

A particular class of integral membrane proteins, the tail-anchored proteins (TA proteins), can only be processed posttranslationally by being targeted to either the ER membrane or the mitochondrial outer membrane (MOM) via a single hydrophobic membrane-anchor sequence, which is located very close to the C-terminus of the polypeptide chain (Kutay et al., 1993). The length of the hydrophobic sequence, as well as the number of positively charged amino acids that flank it, determine where (ER or MOM) the TA protein will be inserted (Wattenberg and Lithgow, 2001). With the exception of TA proteins destined for the MOM, which are targeted there directly from the cytoplasm (Borgese et al., 2001), all the rest must get inserted first into the ER and then transferred to their final destination by vesicular transport (Jantti et al., 1994; Kutay et al., 1995; Linstedt et al., 1995; Pedrazzini et al., 1996). A variety of enzymatic and regulatory roles in cellular metabolism (cytochrome b5), apoptosis (Bcl-2), protein localization (Sec6), and membrane trafficking (SNARE complex proteins) are attributed to TA proteins. By studying the function of each individual TA protein, multiple insertion pathways have been discovered.

\subsubsection{ER-targeting insertional pathways}

Insertion to the ER membrane can happen in a cotranslational or posttranslational mode. Chaperone-mediated pathways are used for cotranslational integration and can be mediated either by signal recognition particle (SRP) and Sec61 protein translocation complex, with GTP hydrolysis, (Abell et al., 2004) or by ATP-dependent Hsc70/Hsp40 chaperones (Abell et al., 2007). The SRP-dependent pathway is mainly utilized by integral membrane proteins that have an N-terminal membrane-targeting signal. SRP is a soluble ribonucleoprotein complex that recognizes the targeting signal of the nascent polypeptide chain, while it is still found in complex with the ribosome. It shields the polypeptide and targets to the ER mem- 
brane by insertion first into the Sec61 translocation channel (Rabu et al., 2009). An exception to the SRP pathway mode was discovered for two TA proteins, synaptobrevin 2 (also known as vesicle-associated membrane protein 2) and Sec61 $\beta$, where the pathway is mediated in a posttranslational step. The key feature of SRP recognition in these cases was shown to be a stretch of hydrophobic residues in the targeting signals of the proteins (Abell et al., 2004).

Much less understood is the Hsc70/Hsp40-mediated pathway. Two lines of evidence indicate that these two chaperones can mediate the biogenesis of TA proteins in vitro (Abell et al., 2007; Rabu et al., 2008), but the exact in vivo pathway is unknown. A broadly utilized posttranslational pathway is driven by the cytosolic ATPase arsA arsenite transporter, ATP-binding, homolog 1 (bacterial) (TRC40; previously described as ASNA1). The pathway requires the interaction of the transmembrane domain (TMD) of a TA protein with a soluble cytosolic protein and the formation of a TMD recognition complex (TRC). This cytosolic factor was found to be TRC40 (Stefanovic and Hegde, 2007). The TRC pathway is a highly ATP-dependent process, which drives consecutive cycles of binding and release of TRC40 from membrane targets competent for TA protein insertion. They proposed the hypothesis that localization of TRC40 on membranes is a transient, ATP-dependent process, which aims to deliver the TA proteins to a receptor. The free cytosolic concentration of TRC40 was detected to be higher relative to SRP, but TRC40 was not found to interact with ribosomes. These facts led the authors to suggest that although TRC40 is more abundant in the cytosol, it can not interfere with nascent polypeptides which are still in association with the ribosome. Cotranslational arrest of TMD targets should happen only by SRP-mediated pathway, whereas posttranslational should be directed by TRC40 (Figure 1.4). Therefore, it was concluded, that these two pathways are distinct and never compete for the same targets (Stefanovic and Hegde, 2007). It is also possible that proteins that have "lost" the chance to get arrested by the SRP pathway use the TRC pathway as an alternative.

Further studies aiming to understand the posttranslational mechanism of membrane protein insertion, led to the identification of another cytosolic heterotrimeric complex, composed of Bag6/Ubl4A/TRC35 (Mariappan et al., 2010). This complex 

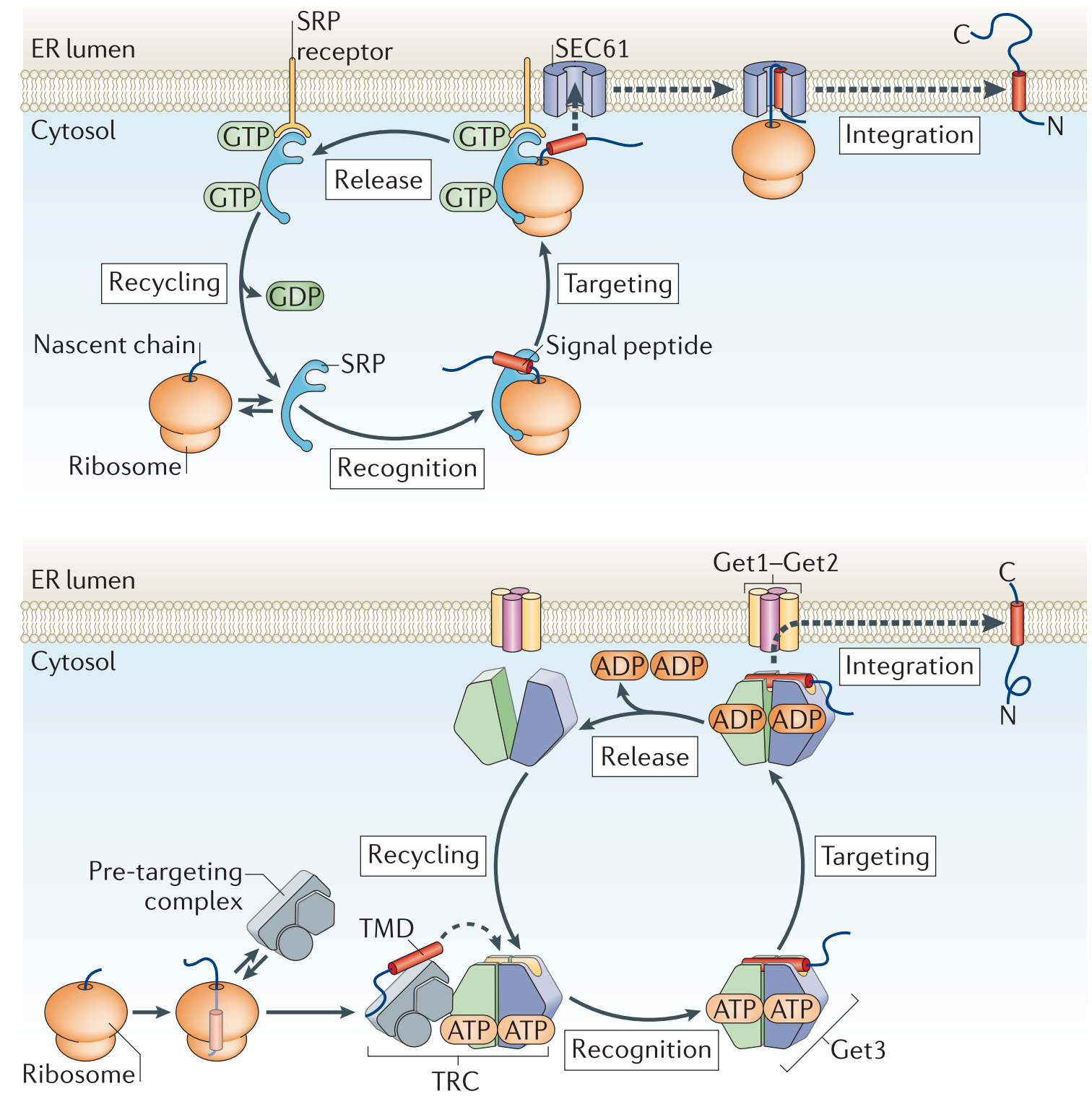

Figure 1.4: The two main membrane insertional pathways. Illustration representing the cotranslational (upper) and the posttranslational (lower) mode of membrane protein insertion to the ER membrane. The SRP/Sec61 pathway is utilized mostly by non-TA proteins, starting during their translation, with the exception of synaptobrevin 2 and Sec61 $\beta$. Tail-anchored proteins display membrane-targeting signals very close to the C-terminus that only after termination of translation exit the ribosome, and then the protein can be recruited to the insertional pathway. The nascent polypeptide chain is "arrested" by the pre-targeting complex (Bag6/Ubl4A/TRC35) and then presented to the cytoplasmic factor TRC40, which through an ATP-dependent process delivers the TA protein to the ER receptor (Get1-Get2). (Figure adapted from (Hegde and Keenan, 2011).) 
acts upstream of TRC40, capturing the TMD of the newly synthesized peptide, as it exits the ribosome, and then passing the TA protein to TRC40 (Mariappan et al., 2010).

Lately, the ER specific receptor of TA proteins has been identified to be the tryptophan-rich basic protein (WRB) (Vilardi et al., 2011). It interacts with the associated TRC40/TA protein complex via a coiled-coil domain in its structure. As TRC40/TA complex arrives at the ER membrane, TRC40 delivers the TA protein to WRB. More light was shed on the TRC pathway, when the receptor partner of WRB on the ER membrane was discovered (Yamamoto and Sakisaka, 2012). Identified by mass spectrometry, calcium-signaling modulating cyclophilin ligand (CAML), is composed of a cytoplasmic domain and three transmembrane domains. It forms a receptor complex together with WRB on the ER membrane. In cell in vitro assays, the transmembrane domain of CAML was shown to compete with the coiled-coil domain of WRB for binding to TRC40, but presence of both proteins was required for TRC40 binding. Many questions on this pathway remain open. How exactly CAML and WRB compete for the interaction with TRC40, what is the order of interaction of TRC40 with the heterodimeric receptor or how exactly the intermolecule interaction between TRC40 and WRB is mediated, are all questions which remain to be investigated.

Similar to the mammalian TRC pathway, the GET pathway for TA protein insertion into the ER is specific to yeast (Saccharomyces cerevisiae). The GET pathway has been characterized by (Schuldiner et al., 2008) and (Wang et al., 2011). According to this mechanism, the nascent TA polypeptide binds to the Sgt2/Get4/Get5 cytosolic complex and, through ATP hydrolysis, is transferred to Get3, the S. cerevisiae homolog of TRC40. Get4 and Get5 are the yeast homologs of Ubl4A and TRC35, respectively. Get3 presents the TA protein substrate to the heteromeric Get1/2 receptor on the ER and interacts transiently with it. The coiled-coil domain of Get1 interacts with Get3, similar to the interaction of WRB with TRC40 (Stefer et al., 2011). ATP binding to Get3 facilitates its release and initiates another round of TA protein targeting. 


\subsubsection{Predictions on the profile of the membrane-target sequence}

A bioinformatics screen (Kalbfleisch et al., 2007), was the first attempt to identify similarities between predicted single transmembrane domains of known human TA proteins and other unknown TA proteins. Although, no common pattern was identified, the findings confirmed previous biochemical data. The level of hydrophobicity and the length of the transmembrane segment of TA proteins targeting the MOM may be lower and shorter than for TA proteins targeting the ER. Three or more positively charged amino acids flanking this hydrophobic segment are required for MOM targeting whereas less are needed for ER targeting (Borgese et al., 2001; Kuroda et al., 1998). Apparently, TA proteins that do not contain MOM-specific targeting information are inserted into the ER, which is more prone to accept transmembrane segments of different lengths and sequences followed by a C-terminal polar region of varying sizes and charges. Perhaps the most interesting finding of this bioinformatics screen was its prediction that human otoferlin is a TA protein; however, this has not yet been demonstrated biochemically.

\subsubsection{Tryptophan-rich Basic Protein - WRB}

In an effort to understand the pathogenesis of the congenital heart disease (CHD) that occurs in more than $40 \%$ of patients with Down syndrome, a functional mapping study identified the $W R B$ gene inside the $C H D$ gene locus in chromosome 21 (Egeo et al., 1998). The WRB gene produces two transcripts (1.4 kb and 1.6 $\mathrm{kb}$ ) and encodes a 174 amino acid tryptophan-rich basic protein, WRB (C-terminus rich in tryptophans and prolines). The evaluation of the WRB tissue expression was performed by Northern blot analysis on human tissues and WRB transcripts were detected in the brain, heart, lung, liver, skeletal muscle, pancreas, kidney, and placenta. Nevertheless, no further study was conducted in order to investigate a possible implication of WRB in CHD pathology.

Additional knowledge about WRB, resulted from an insertional mutagenesis screen for visual deficits in zebrafish (Gross et al., 2005). Of all the mutants generated, one particular was found to carry mutation inside the pwi gene, the zebrafish homolog of WRB. The pwi mutant displayed extensive acellular regions in the photorecep- 
tor area of the retina (outer plexiform layer-OPL) (Gross et al., 2005). Further extensive analysis of the pwi zebrafish mutant, showed that this gene also caused visual, hearing, and balance deficits. When mutated, the Wrb protein affected the swimming behavior and posture of the fish. As far as the auditory sensory cells are concerned, the kinocilia of the lateral line neuromast cells were shortened, likely affecting the mechanotransduction process of the neuromasts, as indicated by the inability of FM dye labeling of the stereocilia of the sensory cells. The mutation further caused hearing deficits, reflected as lack of acoustic startle response and severely reduced microphonic potential. The synaptic activity between the photoreceptors and the bipolar neurons was reduced, as measured by electroretinography. Pinball immunoreactivity appeared generally in the OPL, at the site of photoreceptor-bipolar cell synapses. In the fish, pwi mutation caused mislocalization of the Rab3C synaptic vesicle marker (from the basolateral synaptic region to the apical site), suggesting a possible role of this protein in synaptic development (Lin et al., unpublished). The possible implication of WRB in the pathogenesis of CHD has been additionally supported by a study in medaka fish (Oryzias latipes) (Murata et al., 2009), but this work was mostly focused on examining the effect of CHD in heart formation during development.

In conclusion, the most solid evidence for the function of the tryptophan-rich basic protein can be summarized into two points: WRB is part of the receptor of TA proteins on the ER (Vilardi et al., 2011; Yamamoto and Sakisaka, 2012), and deletion of wrb gene in zebrafish causes hearing and visual defects (Lin et al., unpublished). Based on the prediction (Kalbfleisch et al., 2007) that otoferlin, which is likely the main calcium sensor at the IHC ribbon synapse, is a TA protein, a new hypothesis could be created. Assuming the posttranslational regulation and trafficking of otoferlin by the tryptophan-rich basic protein, which is the implication of WRB in hearing?

\subsection{RNA Interference - Mechanism and Applications}

RNA interference (RNAi) comprises a posttranscriptional mechanism of gene silencing that is conserved in vertebrates (Caplen et al., 2001; Elbashir et al., 2001) and 


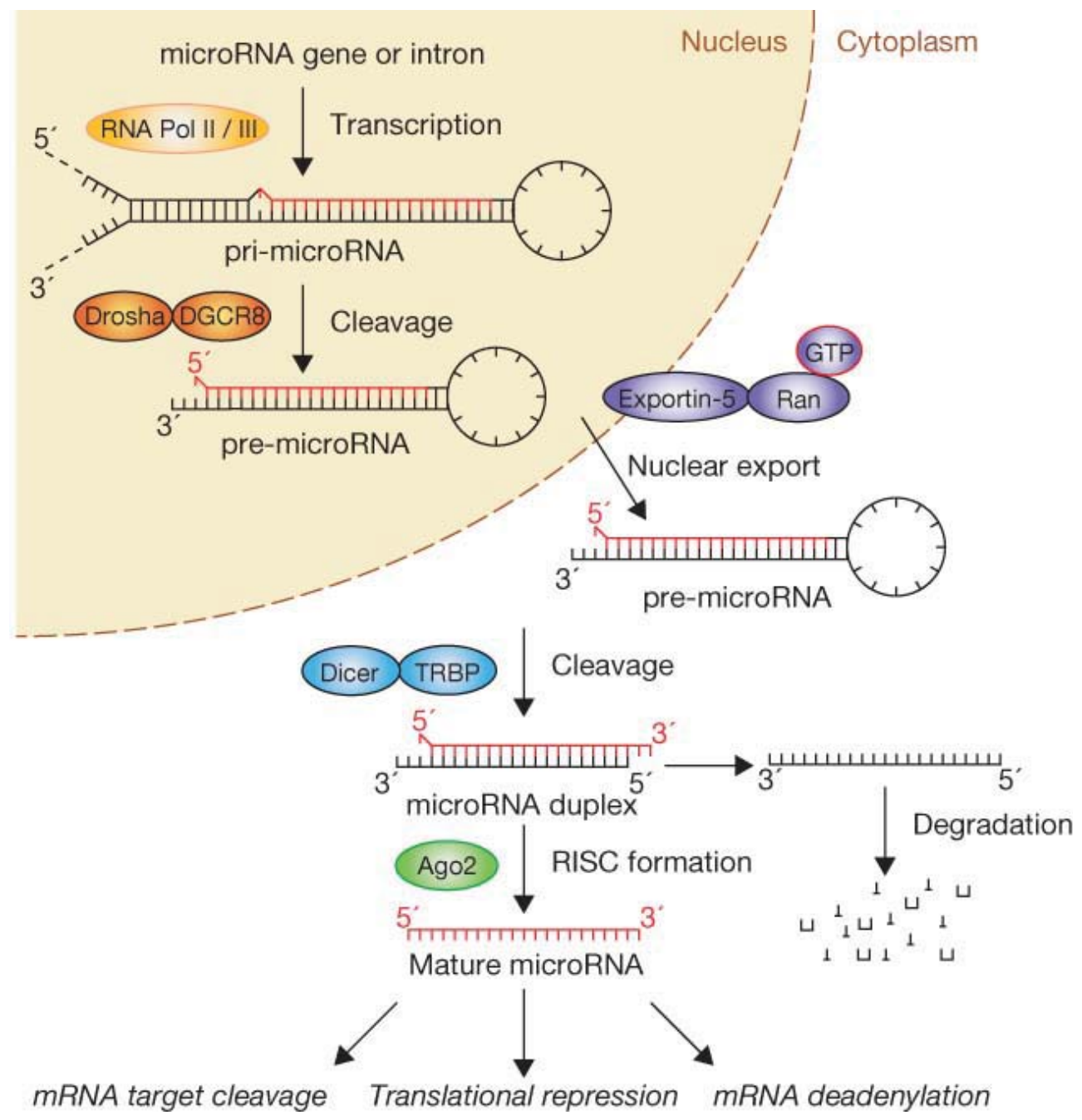

Figure 1.5: miRNA biogenesis pathway. miRNAs are non-coding RNA molecules, transcribed either from independent miRNA genes or from introns of protein-coding transcripts. The primiRNA molecule (> 100 nucleotides) forms multiple hairpins and bulges, and often contains sequences of multiple miRNAs. Two important modification steps take place during the biogenesis of an miRNA, i.e., the catalysis step by Drosha (an RNase III enzyme) inside the nucleus and the final processing step by Dicer inside the cytoplasm. At the end of each step, the precursor miRNA becomes shorter in length until Dicer yields an 21-bp double-stranded miRNA molecule with an unpaired 2-nucleotide 3' overhang. Incorporation of the miRNA duplex into the ribonucleoprotein complex (RISC) is the defining step for silencing. During this step is determined which of the two strands will be the mature miRNA. Argonaute (AGO) proteins 1-4, main components of the RISC, guide the endonucleolytic cleavage or translational repression or deadenylation of the target mRNA. (Figure modified from (Winter et al., 2009).) 
invertebrates (Montgomery and Fire, 1998). The effector molecules are short 21-23 nucleotide double-stranded small interfering RNAs (siRNAs), resulting from multiple intracellular modifications. Originally, RNAi was recognized as an endogenous regulatory pathway that protects the host organism from viral genomes and transposons. Soon, this mechanism received enormous attention with the discovery of miRNAs, that are implicated in the pathogenesis of several diseases, e.g., cancer, metabolism, and neurodegeneration, through the regulation of gene expression.

Gene silencing (knock-down) has become an attractive tool to manipulate the expression of genes. Multiple RNAi inducer molecules (siRNA, shRNA, miRNA) and different viral vector (adeno-associated, adenovirus, lentivirus, retrovirus) approaches have been developed and used widely in mammalian systems. The various strategies of silencing aim to mimic the endogenous RNAi pathway (shown in Figure 1.5) in order to achieve an efficient, long-lasting effect, minimizing the chances of cellular toxicity and off-target effects (for review see (Liu and Berkhout, 2011).)

The previously described findings about IHC function in the pachanga mouse mutant, suggested that a relationship between protein levels and physiological function might exist (Pangršič et al., 2010). Modification of otoferlin protein levels may result in a variety of physiological responses. The approach of gene silencing is one possible method to manipulate the levels of otoferlin.

\subsection{Aims of this study}

While current research has started to elucidate the role of otoferlin at the IHC ribbon synapse, its precise function(s) are not yet fully understood. Multiple indications suggest to be the main calcium sensor, participating in a later step of exocytosis. The levels of the protein at the synapse seem to be critical for its functionality, while in parallel, evidence on the localization of otoferlin at non-synaptic sites, might suggest an extra role in endosomal/Golgi trafficking.

The knowledge about the pinball-wizard mutation in zebrafish sets new questions about the role of this non-synaptic protein into the auditory system. It is quite pos- 
sible that tryptophan-rich basic protein has an indirect role in hearing and rather governs the fate of otoferlin and/or of other TA proteins. If the biogenesis of otoferlin is regulated by the classical posttranslational membrane-insertion pathway of TRC40, then more light can be shed on the regulation and turnover of otoferlin in IHCs.

The goal of this thesis is mainly to characterize the physiological function of WRB in a loss of function mutant. Moreover, in a preliminary attempt, a gene silencing strategy was designed, for delivery of miRNA-like silencing molecules by adeno-associated viruses in the developing mouse otocyst.

Using a conditional knockout mouse line, I applied system-, cell physiology, and morphological approaches to understand the phenotype of the wrb deletion. For the designing of the silencing strategy, I used molecular and cell biology, in combination with viral transduction in the developing cochlea. 


\section{2}

\section{Materials AND Methods}

\section{$2.1 \quad$ Materials}

\subsubsection{Chemicals}

The chemicals used in this study were purchased from BD Biosciences (Germany), Carl ROTH, Fluka Analytical (SIGMA-ALDRICH Laborchemikalien GmbH Seelze, Germany), Invitrogen (Darmstadt, Germany), MERCK (Darmstadt, Germany) and ROCHE (Mannheim, Germany). Amphotericin B, Streptomyces sp., cat. No. 171375, 100 mg was purchased from Calbiochem (Merck KGaA, Darmstadt, Germany).

\subsubsection{Enzymes, kits and bacterial strains}

All restriction enzymes, DNA and Protein Ladders were purchased from FermentasThermo Fischer Scientific GmbH (Schwerte, Germany). For DNA gel extraction reactions, purification of digestion reactions, as well as for proliferations of DNA plasmids, kits from ZYMO Research (Freiburg, Germany), Peqlab Biotechnologie GmbH (Erlangen, Germany), and Macherey-Nagel GmbH \& Co.KG (Germany) were used. Amplification of DNA plasmids was performed in Escherichia coli (E. coli) XL1-Blue, SURE (Stratagene, Agilent Technologies GmbH \& Co.KG, Waldbronn), and DH5 $\alpha$ (Invitrogen) bacterial strains.

\subsection{3 miRNA sequences and oligomers}

Primer design and all the major cloning steps were first simulated and confirmed using GENtle software (University of Cologne, Germany). Multiple miRNA se- 
quences were designed against the mRNA of $M$. musculus otoferlin transcript variant 1 (GenBank accession no. NM_001100395.1), using the algorithms BLOCK-iT ${ }^{T M}$ RNAi Designer (Invitrogen) and siRNA Wizard v3.1. The final 21mer nucleotide sequences were tested through a BLAST search to ensure specificity for the target mRNA. The previously mentioned sequences, as well as all the following described oligomers, were chemically synthesized by the AGCT Lab in the DNA Core Facility of the Max Planck-Institute of Experimental Medicine in Göttingen.

\subsubsection{Sequencing reactions and viral vector production}

Sequencing of the DNA constructs was performed and validated by the AGCT Lab. The viral vector AAV-HBA-EWB, as well as all the viruses used in this study, were produced by Dr. Sebastian Kügler from the Viral Vectors Lab, Department of Neurology, at the University Medicine of Göttingen.

\subsubsection{Buffers}

\section{Immunohisto-, immunocyto-chemistry}

PBS (Phosphate Buffer Saline): $137 \mathrm{mM} \mathrm{NaCl}, 10 \mathrm{mM} \mathrm{Na}{ }_{2} \mathrm{HPO}_{4}, 2.7 \mathrm{mM}, \mathrm{pH}$ 7.4. PB (Phosphate Buffer): $240 \mathrm{mM} \mathrm{Na}_{2} \mathrm{HPO}_{4} \mathrm{H} 2 \mathrm{O}$.

GSDB (Blocking solution): goat serum, 0.3\% Triton X-100, 240 mM PB, 4 M NaCl. Wash buffer: $20 \mathrm{mM} \mathrm{PB}, 0.3 \%$ Triton X-100, $450 \mathrm{mM} \mathrm{NaCl}$.

Blocking buffer: $10 \%$ goat serum, $0.3 \%$ Triton X-100 in PBS pH 7.4.

4\% Paraformaldehyde: From a 37\% initial stock (Carl ROTH), the final working concentration that was used was $4 \%$ in PBS.

Mowiol Mounting Medium: Dissolve $2.4 \mathrm{~g}$ Mowiol 4-88 (Carl ROTH) into $6 \mathrm{~g}$

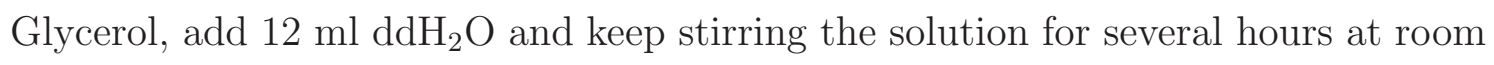
temperature. Add $0.2 \mathrm{M}$ Tris $\mathrm{pH} 8.5$ and heat the mixture for $1-2 \mathrm{~h}$ at $50{ }^{\circ} \mathrm{C}$. When Mowiol is dissolved, the solution is centrifuged at $500 \mathrm{x}$ g for $15 \mathrm{~min}$, add 2.5\% DABCO (Carl ROTH) and aliquoted.

DAPI: Solution prepared according to manufacturer instructions (10 mg, cat. No. D1306, Invitrogen). 


\section{Protein lysates}

RIPA buffer (for mammalian cells): $50 \mathrm{mM}$ Tris- $\mathrm{HCl} \mathrm{pH}$ 7.4, $150 \mathrm{mM} \mathrm{NaCl}$, $2 \mathrm{mM}$ EDTA, 1\% NP-40, 0.1\% SDS. On the day of use $1 \mathrm{mM}$ PMSF and phospatase/protease inhibitors cocktail tablets were added (PhosSTOP and complete ULTRA tablets, Mini, EDTA-free, ROCHE), according to the manufacturer instructions.

\section{$S D S-P A G E$}

2x Protein Sample buffer: 125 mM Tris-HCl pH 6.8, 4\% SDS, 150 mM DTT, 20\% Glycerol, 0.01\% Bromophenol blue.

Running buffer: 25 mM Tris-HCl, $192 \mathrm{mM}$ Glycine, 0.1\% SDS.

Transfer buffer: 25 mM Tris-HCl, 192 mM Glycine, 20\% Methanol.

TBS: 200 mM Tris- $\mathrm{HCl} \mathrm{pH}$ 7.6, 1,368 mM NaCl.

TBST: 200 mM Tris-HCl, 1,368 mM NaCl, 0.1\% Tween 20 .

\section{Molecular Biology}

11.1x PCR Buffer: 45 mM Tris- $\mathrm{HCl}$ pH 8.8, $11 \mathrm{mM}$ ammonium sulphate, $4.5 \mathrm{mM}$ $\mathrm{MgCl}_{2}, 6.7 \mathrm{mM}$ 2-mercaptoethanol, $4.4 \mu \mathrm{M}$ EDTA pH 8.0, $1 \mathrm{mM}$ of each dNTP, $113 \mu \mathrm{g} / \mathrm{ml}$ BSA.

10x Oligo Annealing Buffer (used in annealing reactions of miRNA oligomers):

100 mM Tris- $\mathrm{HCl}$ pH 7.5, 10 mM EDTA pH 8.0, 1,000 mM NaCl.

10x TE Buffer: 100 mM Tris-HCl pH 7.5, 10 mM EDTA pH 8.0.

50x TAE DNA Electrophoresis buffer: 2,000 mM Tris-HCl, 50 mM EDTA.

DNA loading dye: 50\% Glycerol, 1 mM EDTA pH 8.0, 0.15\% Bromophenol blue.

\section{Tissue cultures}

2x HBS (HEPES buffered saline): $280 \mathrm{mM} \mathrm{NaCl}, 10 \mathrm{mM} \mathrm{KCl}, 1.5 \mathrm{mM} \mathrm{Na}_{2} \mathrm{HPO}_{4}$, $12 \mathrm{mM}$ Dextrose, $50 \mathrm{mM}$ HEPES, pH 7. Sterile filtered through $0.22 \mu \mathrm{m}$ filter.

\section{Cell physiology}

HEPES-buffered Hanks balanced salt solution: $5.36 \mathrm{mM} \mathrm{KCl,} 141.7 \mathrm{mM} \mathrm{NaCl}, 1$ $\mathrm{mM} \mathrm{MgCl}_{2} 6 \mathrm{H}_{2} \mathrm{O}, 0.5 \mathrm{mM} \mathrm{MgSO}{ }_{4} 7 \mathrm{H}_{2} \mathrm{O}, 10 \mathrm{mM}$ HEPES, pH 7.2, 290 mOsm. Before dissection of the organ of Corti $2 \mathrm{mg} / \mathrm{ml}$ glucose and $0.5 \mathrm{mg} / \mathrm{ml} \mathrm{L-glutamine}$ was added.

Modified Ringers solution (extracellular solution): $2.8 \mathrm{mM} \mathrm{KCl,} 110 \mathrm{mM} \mathrm{NaCl}, 1$ 
mM $\mathrm{MgCl}_{2} 6 \mathrm{H}_{2} \mathrm{O}, 35 \mathrm{mM}$ TEA-Cl, $10 \mathrm{mM}$ HEPES, $1 \mathrm{mM} \mathrm{CsCl}, 2 \mathrm{mM} \mathrm{CaCl} 2, \mathrm{pH}$ 7.2, 290 mOsm. Before recordings $2 \mathrm{mg} / \mathrm{ml}$ glucose was added.

Intracellular recording solution for perforated patch: $130 \mathrm{mM}$ Cs-gluconate, $10 \mathrm{mM}$ TEA-Cl, 10 mM 4-AP, 1 mM $\mathrm{MgCl}_{2} 6 \mathrm{H}_{2} \mathrm{O}, 10$ mM HEPES, pH 7.2 adjusted with $\mathrm{HCl}, \sim 290 \mathrm{mOsm}$. On the day of the experiment $300 \mu \mathrm{g} / \mathrm{ml}$ amphotericin B was added.

Amphotericin stock solution: A stock solution of Amphotericin $50 \mu \mathrm{g} / \mu \mathrm{l}$ in Dimethyl Sulfoxide (DMSO, SIGMA-ALDRICH) was prepared every 3 days and $300 \mu \mathrm{g} / \mathrm{ml}$ were diluted always fresh into $0.5 \mathrm{ml}$ of intracellular solution. The solution was sonicated thoroughly, protected from light and kept on ice until use.

\section{Genomic DNA genotyping}

Genomic Mouse Tail Buffer (GMT): $10 \mathrm{mM}$ Tris pH 8.5, $50 \mathrm{mM} \mathrm{KCl,} 1.5 \mathrm{mM}$ $\mathrm{MgCl}_{2}, 0.01 \%$ gelatin, $0.45 \%$ igepal $630,0.45 \%$ Tween-20. Freshly added in each $\mathrm{PCR}$ reaction tube $100 \mu \mathrm{g} / \mathrm{ml}$ proteinase $\mathrm{K}$.

\subsubsection{Antibodies}

\section{Primary}

- mouse anti-CtBP2 aa. 361-445, IgG1, cat. No. 612044 (BD Biosciences)

- mouse anti-Otoferlin [clone13A9], cat. No. ab53233 (Abcam, Germany)

- rabbit anti-GFP serum cat. No. 598 (MoBiTec, Göttingen, Germany)

- rabbit anti-glutamate receptor 2 \& 3 cat. No. AB 1506 (Millipore, Merck)

- rabbit anti-VGLUT3 aa. 543-601, cat. No. 135203 (Synaptic Systems)

- rabbit anti- $\alpha$ tubulin aa. 443-449, cat. No. 302203 (Synaptic Systems)

- rabbit anti-TRC40 was a kind gift from Dr. Fabio Vilardi (Favaloro et al., 2010) 


\section{Secondary}

All the secondary antibodies were Alexa Fluor IgG $(\mathrm{H}+\mathrm{L})$, Molecular Probes (Invitrogen)

- 488 goat anti-rabbit cat. No. A11008

- 488 goat anti-mouse cat. No. A11001

- 568 goat anti-mouse cat. No. A11004

- 568 goat anti-rabbit cat. No. A11011

- 633 goat anti-rabbit cat. No. A21070

- anti-GFP rabbit Alexa Fluor 488 conjugate cat. No. A21311

\subsubsection{Primers}

The primers used for genotyping were:

wt forward: 5'-TTGGTGAGGCTTGTCTCTGGCGATTT -3'

wt reverse: 5'-GTTGATCTTCCTTTCCAGCCTGGCGTAT -3'

dneo forward: 5'-AGTGTACACGCGTACTAGTCTAGCGAA -3'

dneo reverse: 5'-ACTCAAAGGCAACAGCCAAGATAAGCA -3'

The primers used for checking the length of the transcript after WRB recombination were:

wrb exon1 forward: 5'-GTGTTGAGCTTCGTGTTCGG-3'

wrb exon2 reverse: 5'-ACGTGCGTTTTGAGCTTGTC-3'

wrb exon5 reverse: 5'-GCTCAATGGCGGGTGTAGAA-3'

parvalbumin forward: 5' -TGCAGGATGTCGATGACAGAC-3'

parvalbumin reverse: 5'-TCAGGCCCACCATCTGGA-3'

\subsubsection{Animals}

Animal handling was in accordance with the national animal care guidelines and all experiments were reviewed and approved by the animal welfare committees of the University of Göttingen and the State of Lower Saxony. 


\subsubsection{Mouse lines}

\section{"pinball-wizard (Pwi)"}

The conditional mouse line with floxed the WRB (tryptophan-rich basic protein) gene was kindly offered to us by Professor David Corey (Harvard Medical School) and Dr. Shuh-Yow Lin (UC San Diego). Briefly, it was produced as follows: the wild-type WRB genomic locus was targeted by the FRT/loxP recombination system. The insertion of a neomycin (neo) cassette, flanked by 2 Flp (flippase) Recombination Target (FRT) sites, served as a selection marker of those embryonic stem (ES) cells which had properly uptaken the target construct. These ES with resistance in the antibiotic neomycin were further selected to be injected into the blastocyst of a pregnant mouse. The offspring with the targeted gene were crossed with a flippase-expressing mouse line to remove the neo cassette by FRT site-directed recombination. In the conditional line, the exons 2-4 of WRB are targeted for excision. The line is of $129 \mathrm{SVEV}$ background. It was kept either in homozygous ( $\mathrm{Pwi}{ }^{\mathrm{fl}} / \mathrm{fx}$ ) or heterozygous $\left(\mathrm{Pwi}^{\mathrm{flx} /+}\right)$ breeding for the floxed wrb gene.

\section{"VDCR-CGCT"}

The Cre expressing reporter mouse line $V D C R-C G C T$, was a kind gift from Dr. Sonja Wojcik (Max-Planck Institute for Experimental Medicine, Göttingen). It contained two transgenes, independently inherited from each other; the VDCR, in which a Vglut3 promoter may express the bacterial Cre recombinase gene in those tissues and cells that express VGLUT3 and the CGCT, in which a constitutive promoter $(\mathrm{CAG})$ can only guide the expression of the reporter gene EGFP if homologous Cre recombination removes the stop cassette before EGFP. Due to this design, the EGFP expression is achieved only when Cre recombinase is active and only in tissues that express VGLUT3. The VDCR was active in homozygous or heterozygous state and was abbreviated $\mathrm{VDCR}^{+}$.

The pinball-wizard line was cross-bred with the Vglut3-CRE specific EGFP re-

porter line VDCR-CGCT. Heterozygous or homozygous $W r b$ floxed, $\mathrm{Pwi}{ }^{\mathrm{Ax} /+}$ or 
$\mathrm{Pwi}^{\mathrm{flx} / \mathrm{flx}}$, were initially mated with $\mathrm{VDCR}^{+} \mathrm{CGCT}^{+}$animals, to obtain all three transgenes in the same mouse line. The following breedings were performed: $\mathrm{Pwi}^{\mathrm{flx} /+}$ $\mathrm{VDCR}^{+} \mathrm{CGCT}^{+}$mice were mated with $\mathrm{Pwi}^{\mathrm{flx} / \mathrm{flx}} \mathrm{VDCR}^{-} \mathrm{CGCT}^{+}$mice. The offspring used were: $\mathrm{Pwi}^{\mathrm{flx} / \mathrm{flx}} \mathrm{VDCR}^{+} \mathrm{CGCT}^{+}(\mathrm{KO}), \mathrm{Pwi}^{\mathrm{flx} / \mathrm{flx}} \mathrm{VDCR}^{-} \mathrm{CGCT}^{+}$or (control) and $\mathrm{Pwi}^{+/+} \mathrm{VDCR}^{+} \mathrm{CGCT}^{+}$(control). Pwilliffx $\mathrm{VDCR}^{-} \mathrm{CGCT}^{+}$or ${ }^{-}$control mice carrying $\mathrm{CGCT}^{+}$or $\mathrm{CGCT}^{-}$were used and throughout the thesis they will be symbolized as $\mathrm{Pwi}^{\mathrm{flx} / \mathrm{fx}} \mathrm{VDCR}^{-} \mathrm{CGCT}^{+}$. For all the experiments with the pinball-wizard line, animals of up to two months of age were used.

\section{Otoferlin heterozygous}

Otoferlin heterozygous, Otof ${ }^{+/-}$animals resulted from the breeding of Otoferlin knock-out (generated by Dr. Ellen Reisinger) with wild-type C57BL/6J. For the cell physiology experiments on Otof $^{+/-}$, control measurements were performed on wild-type C57BL/6J animals.

\section{Viral injected animals}

Embryonic transuterine otocyst injections were performed in embryonic day 11.5 (E11.5) embryos of wild-type CD1 female pregnant mice, previously mated with CD1 males.

All new born animals were genotyped shortly after birth and before each experiment to ensure their identity and plan the downstream experiments accordingly.

\section{$2.2 \quad$ Methods}

\subsubsection{Cloning procedures}

Molecular cloning was performed according to the standardized protocols described in Molecular Cloning Laboratory Manual, 2nd edition (Joseph Sambrook and David W. Russell, 1989). 


\subsubsection{Cloning of the microRNA expressing vector}

The goal was to create a vector, which expresses a miRNA oligomer and to report this expression by a fluorescent marker. For that, the commercially available pEGFP-C1 vector (Clontech, TaKaRa Bio Company) was used as the backbone and the microRNA vector preparation was performed in two consecutive steps. The final product of this procedure was abbreviated pEGFP-C1_miRNA.

\section{Step 1: 'microtemplate' cassette}

First, a microtemplate cassette was introduced into the pEGFP-C1 backbone, as Figure 2.1 shows. The microtemplate cassette sequence was:

5 '-TCGAGCTTAACTGGAGGCTTGCTGAAGGCTGTATGCTGCGGCCGC

CAGGACACAAGGCCTGTTACTAGCACTCACATGGAACAAATGGCCA -3’

TCGAG: XhoI restriction enzyme overhang

CT: linker from pEGFP-C1 vector

TAA: stop codon of EGFP

CTGGAGGCTTGCTGAAGGCTGTATGCT: 5' flanking region

GCGGCCGC: NotI restriction enzyme

CAGGACACAAGGCCTGTTACTAGCA

CTCACATGGAACAAATGGCC: 3' flanking region

A: HindIII restriction enzyme overhang 


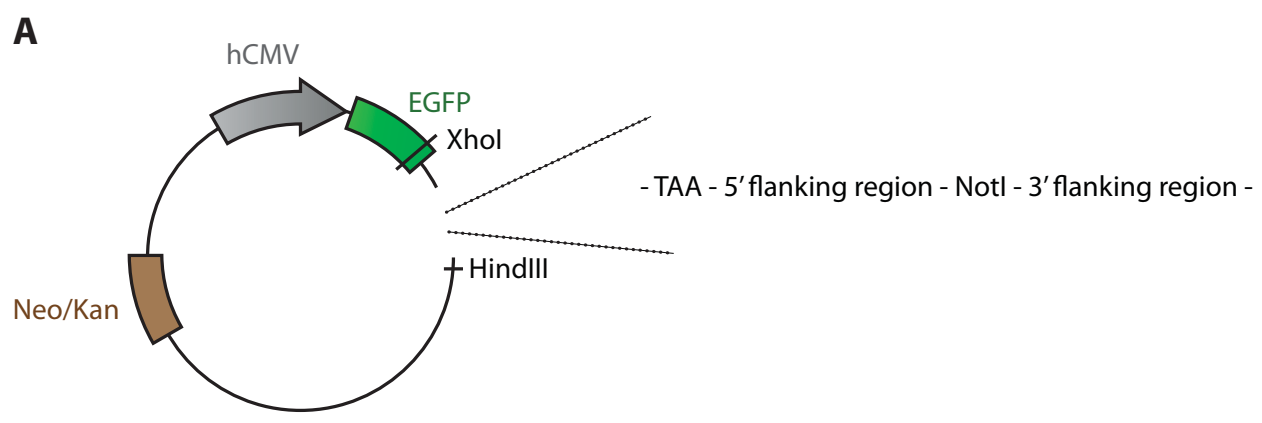

Figure 2.1: The pEGFP-C1 backbone. Cartoon illustrates the pEGFP-C1 backbone. The microtemplate casette was introduced between the restriction sites XhoI and HindIII, downstream of EGFP.

This cassette was introduced in two separate parts. First, the pEGFP-C1 vector was digested with XhoI and HindIII restriction enzymes for $1 \mathrm{~h}$ at $37^{\circ} \mathrm{C}$, followed by $20 \mathrm{~min}$ incubation at $80^{\circ} \mathrm{C}$ for inactivation of the enzymes. Afterwards, the backbone was dephosphorylated with alkaline phosphatase enzyme for $10 \mathrm{~min}$ at $37^{\circ} \mathrm{C}$ and subsequently incubated for $5 \mathrm{~min}$ at $80^{\circ} \mathrm{C}$ for inactivation of the enzyme. The product was then loaded on a $1 \%$ agarose gel from where the band of interest was isolated and processed for DNA extraction and purification. The clean product was eluted in $0.1 \mathrm{x}$ TE Buffer. The first half of the microtemplate cassette was then ligated with the digested backbone for $2 \mathrm{~h}$ at room temperature. Phosphorylation of the endings and oligomer annealing reactions were performed as described later.

\section{first half of microtemplate \\ 5 '-TCGAGCTTAACTGGAGGCTTGCTGAAGGCTGTATGCTGCGGCCGCGAGAA -3'}

Afterwards the pEGFP-C1 vector containing the first half of the microtemplate was digested once more with NotI and HindIII restriction enzymes for $1 \mathrm{~h}$ at $37^{\circ} \mathrm{C}$ with $1 \mathrm{x}$ Tango buffer and then for an additional $1 \mathrm{~h}$ with $2 \mathrm{x}$ Tango buffer and purified as described previously. The second half of the microtemplate cassette was then ligated with the digested backbone. The resulting pEGFP-C1_microtemplate vector was sequenced to confirm the correct insertion of the microtemplate. 


\section{second half of microtemplate}

5 '-GGCCGCCAGGACACAAGGCCTGTTACTAGCACTCACATGGAACAAATGGCCA -3'

The termini of $50 \mu \mathrm{M}$ forward and $50 \mu \mathrm{M}$ reverse strand of each oligomer were phosphorylated using 10 Units of T4 PNK enzyme, T4 PNK Buffer A 10x and $10 \mathrm{mM}$ ATP for $30 \mathrm{~min}$ at $37^{\circ} \mathrm{C}$. An additional $5 \mathrm{~min}$ incubation time at $75^{\circ} \mathrm{C}$ was used for deactivation of the enzyme. Then they were heated at $95^{\circ} \mathrm{C}$ for $4 \mathrm{~min}$ and left to anneal slowly while cooling down at room temperature.

\section{Step 2: miRNA oligomer}

Every miRNA oligomer (from 5' to 3') consisted of: the restriction enzyme NotI overhang, the reverse compliment of the 21 mer miRNA sequence, the miRNA loop sequence, the 1-8 and 11-21 nucleotides of the 21mer sequence and again the restriction enzyme NotI overhang. All of them were phosphorylated and annealed as described before and further subcloned inside the microtemplate cassette of the pEGFP-C1 vector. Digestion of the pEGFP-C1 vector was done by using the NotI restriction enzyme and ligation with the miRNA oligomer was achieved after $2 \mathrm{~h}$ at room temperature. The resulting pEGFP-C1_miRNA vectors were sequenced to confirm the correct insertion of the miRNA oligos.

The miRNA oligos used were:

\section{miRNA1:}

5' -GGCCGCGATCAGAAGTCGCATCCAGTTTGTTTTGGCCACTGAC

TGACAAACTGGACGACTTCTGATGC-3'

miRNA2:

5 ' -GGCCGCGAATTCTTCCATTCTCCCTGTGGTTTTGGCCACTGAC TGACCACAGGGAATGGAAGAATTGC-3' 
scramble1:

5 ' -GGCCGCGTGGTCAAGCGGATATTCCAATGTTTTGGCCACTGAC

TGACATTGGAATCCGCTTGACCAGC-3'

scramble2:

5 '-GGCCGCGTCACTACGACTCAGTTCCAATGTTTTGGCCACTGAC

TGACATTGGAACAGTCGTAGTGAGC-3'

The 21mer miRNA sequences used were:

miRNA1 (3969-3989 bp of NM_001100395.1):

5 '-AAACTGGATGCGACTTCTGAT -3'

miRNA2 (1985-2005bp of NM_001100395.1):

5' -CACAGGGAGAATGGAAGAATT -3'

control sequence 1 (scrambled sequence of NM_001100395.1):

5 '-ATTGGAATATCCGCTTGACCA-3'

control sequence 2 (scrambled sequence of NM_001100395.1):

5'-ATTGGAACTGAGTCGTAGTGA-3'

\subsubsection{Cloning of the AAV1/2 -HBA-EGFP-miRNA vector}

In order to in vivo silence the protein of our interest, a viral vector able to transduce inner hair cells and express the different miRNAs was created. This viral vector expressed the whole microtemplate cassette with the different miRNA oligos tagged to a fluorescent reporter. The backbone AAV1/2-HBA-EWB viral vector was kindly provided by Dr. Sebastian Kügler. It contained inverted terminal repeats (ITRs) of the adeno-associated virus (AAV) serotype 2, the human cytomegalovirus (CMV) enhancer combined with the human actin promoter (HBA), an eGFP (enhanced GFP) reporter protein, a woodchuck posttranscriptional regulatory 
element (WPRE), and a bovine growth hormone (BGH) polyadenylation sequence. It expressed a chimeric capsid from the serotype 1 and 2 .

The backbone, as well as the pEGFP-C1_miRNA vector, were digested with NheI and HindIII restriction enzymes. The bands of interest from the backbone and the miRNA vector were purified from an agarose gel and ligated for $3 \mathrm{~h}$ at room temperature. The ligated vector was then concentrated by ethanol precipitation before amplification into SURE electrocompetent bacteria. The final viral product was sequenced to ensure the correct insertion of the miRNA oligo and amplified in E. coli bacteria.

\subsubsection{Ethanol precipitation of DNA}

The ligated product of the AAV1/2 -HBA-EGFP-miRNA vector was incubated for 2 days in $-20^{\circ} \mathrm{C}$ with $1: 10$ volume of $\mathrm{NaOAc} 3 \mathrm{M}, 1 \mu$ l glycogen and $100 \%$ ethanol. Then, it was centrifuged at $13,000 \mathrm{rpm}$ at $4^{\circ} \mathrm{C}$ for $1 \mathrm{~h}$ and the pellet was washed with $70 \%$ ethanol. The residual ethanol was removed by centrifugation, the DNA pellet was air-dried and was resuspended in $\mathrm{ddH}_{2} \mathrm{O}$.

\subsubsection{Bacterial transformation}

\section{Chemical transformation}

The DNA was amplified in E. coli, DH5 $\alpha$, and XL-1 Blue chemical competent bacterial strains. Generally, the amount of DNA mixed with bacteria was varied. DNA $0.5 \mu \mathrm{l}-5 \mu \mathrm{l}$ was mixed with $50 \mu \mathrm{l}$ bacteria and incubated on ice $30 \mathrm{~min}$. Afterwards, it was transferred at $42{ }^{\circ} \mathrm{C}$ for $2 \mathrm{~min}$ and then placed on ice for additional $2 \mathrm{~min}$. LuriaBroth medium $800 \mu \mathrm{l}$ was added and the bacteria containing the DNA were incubated while shaking for $1 \mathrm{~h}$ at $37^{\circ} \mathrm{C}$ at $250 \mathrm{rpm}$. At the end of the incubation the mixture was centrifuged, most of the supernatant was discarded and the pellet was resuspended in a remaining volume of $30 \mu \mathrm{l}$. The resuspended bacteria were plated on agar antibiotic-specific plates and antibiotic-resistant colonies were let to grow overnight. Next day clones of successfully transformed bacteria were picked 
up and let further grow for $16 \mathrm{~h}$ at $37^{\circ} \mathrm{C}$ at $250 \mathrm{rpm}$. DNA was isolated and purified.

\section{Electrical transformation}

For the amplification of viral vectors the electroporation-competent bacteria cells SURE were used. $50 \mu \mathrm{l}$ were mixed with $0.5-1 \mu \mathrm{l}$ viral plasmid DNA in a pre-chilled cuvette (Gene Pulser $0.1 \mathrm{~cm}$ BIO-RAD). The bacteria were electroporated at the Biorad Gene Pulser II at $\mathrm{V}=1.7 \mathrm{kV}$, to uptake the viral DNA. Immediately after the pulse, the cells were transferred into an eppendorf tube and $800 \mu \mathrm{l}$ Luria Broth medium was added. The mixture was processed the same way as described above and then, it was let grow overnight on agar plates with the antibiotic Carbenicillin $(50 \mathrm{mg} / \mathrm{ml})$. The antibiotic-resistant bacterial colonies were processed as previously described.

\subsubsection{Cell Culture}

Human embryonic kidney (HEK 293T) cells were grown in Dulbeccos modified Eagles medium (DMEM from Invitrogen) supplemented with 10\% fetal calf serum (FCS), $100 \mathrm{U} / \mathrm{ml}$ mixture penicillin/streptomycin and incubated at $37^{\circ} \mathrm{C}$ with $5 \%$ $\mathrm{CO}_{2}$. They were passaged when they reached confluency, normally every 4 days. For this process the medium was removed, a single wash with $5 \mathrm{ml}$ pre-warmed PBS was done and then $2 \mathrm{ml}$ of pre-warmed $\left(37^{\circ} \mathrm{C}\right)$ Trypsin/EDTA solution was added to the culture dish for detaching the cells. The trypsin activity was stopped by addition of DMEM. The cells were carefully transferred to a $15 \mathrm{ml}$ Falcon tube and were centrifuged for $5 \mathrm{~min}$ at room temperature at $2.500 \mathrm{rpm}$. The pellet was resuspended in fresh DMEM and the cells were seeded in new culture dishes (Cell Star Greiner bio-one, Solingen, Germany). They were used until their $20^{\text {th }}$ passage.

\subsubsection{Cell Transfection Methods}

For downstream immunocytochemistry experiments, the cells were grown on coverslips first washed with $70 \%$ ethanol and then coated with $0.01 \%$ poly-L-lysine 
ready-to-use solution (SIGMA-ALDRICH). After $20 \mathrm{~min}$ incubation with poly-Llysine, the solution was removed and cells were plated directly on the coverslips.

\section{Lipofection}

The cells were plated one day prior transfection in 6 -well plates or 24 -well plates in normal DMEM supplemented with $10 \%$ fetal calf serum (FCS) and $100 \mathrm{U} / \mathrm{ml}$ mixture penicillin/streptomycin. One hour before transfection the medium of the cells was changed to Opti-MEM (Invitrogen). For one well of the 24-well plate 0.8-1 $\mu \mathrm{g}$ DNA was diluted in $50 \mu$ l OptiMEM and separately 1-2 $\mu$ l Lipofectamine 2000 (Invitrogen) in $50 \mu \mathrm{l}$ OptiMEM. After $5 \mathrm{~min}$ incubation, the two solutions were mixed and 20 min later the transfection mix was added to the well. For one well of the 6-well plate, $4 \mu \mathrm{g}$ DNA was diluted in $250 \mu \mathrm{l}$ OptiMEM and separately $10 \mu \mathrm{l}$ Lipofectamine 2000 (Invitrogen) in $250 \mu \mathrm{l}$ OptiMEM. For double plasmid transfections 6-well plates were used and the ratio of the two plasmids varied, always keeping a total maximum amount of $8 \mu \mathrm{g}$ DNA and $12 \mu \mathrm{l}$ Lipofectamine per well. The transfection mix was removed $6 \mathrm{~h}$ after and replaced with normal DMEM. Cells were processed $48 \mathrm{~h}$ post-transfection.

\section{Calcium phosphate}

The cells were plated one day prior transfection in 6-well plates or $10 \mathrm{~cm}$ cell culture dishes, waiting for 60-70\% confluency until the transfection. For 1 well of the 6 -well plate, $3 \mu \mathrm{g}$ DNA were diluted with $20 \mu \mathrm{laCl}_{2} 2.5 \mathrm{M}$ solution in a final volume of $85 \mu \mathrm{l}$ (Solution A). The Solution A was added dropwise to $85 \mu \mathrm{l}$ 2x HBS (Solution B). For a $10 \mathrm{~cm}$ cell culture dish the Solution A consisted of

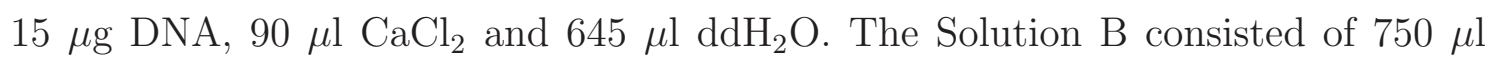
$2 \mathrm{x}$ HBS. The mix of the two solutions was mixed vigorously and left for 2 min at room temperature to allow the $\mathrm{DNA}-\mathrm{CaCl}_{2}$ complexes to form. Then it was added in fresh DMEM medium supplemented with $2 \%$ FCS and finally added to the cells. The transfection mix was removed $4 \mathrm{~h}$ later and replaced with normal DMEM. Cells were processed $48 \mathrm{~h}$ post-transfection. 


\subsubsection{Preparation of protein lysates}

\section{Cell protein lysates}

Transfected cells were processed 24-48 h after transfection. The culture medium was removed from each plate or well and washed with icecold PBS. The cells were collected in RIPA buffer, containing protease and phosphatase inhibitors, with a plastic scraper and further lysed by triturating them three times through a $27 \mathrm{G}$ needle. The lysate was centrifuged for $45 \mathrm{~min}$ at 10,000 rpm and the supernatant was kept for western blot.

\subsubsection{Protein concentration estimation}

Protein concentration of every cell lysate or tissue sample was determined using the Bradford colorimetric protein assay (SIGMA-ALDRICH). Six different dilutions $(0,2.5,5,10,15,20 \mathrm{mg} / \mathrm{ml})$ of $1 \mu \mathrm{g} / \mu \mathrm{l}$ Bovine Serum Albumin (BSA, purified 100x, $10 \mathrm{mg} / \mathrm{ml}$, New England BioLabs Inc.) were prepared, adding RIPA buffer and Bradford Reagent. These standards were prepared in triplicates, measured in a SmartSpec Plus Spectrophotometer (Biorad) and the protein absorbance at 595 nm determined the concentration. From the values, a standard curve of protein concentration was designed. The concentration of the protein was estimated by the standard curve.

\subsubsection{Western Blotting}

Samples were separated in a $8 \%$ (denaturating) Tris-HCl SDS polyacrylamide gel electrophoresis system. The resolving gel consisted of $8-10 \%$ bis-acrylamide (Rotiphorese Gel 30, Roth GmbH, Karlsruhe, Germany), 1 M Tris ( $\mathrm{pH} 8.45$ ), 0.1\% SDS, 10\% glycerol; the stacking gel contained 4\% bis-acrylamide, $1 \mathrm{M}$ Tris ( $\mathrm{pH}$ 8.45), 0.1\% SDS. Ammonium persulfate and TEMED (N, N, N', N'Tetramethylethylenen-diamine) were added for polymerization. 1:5 2x Protein Sample buffer was added to the samples and they were boiled for $5 \mathrm{~min}$. 20-50 $\mu \mathrm{g}$ of protein were loaded to the gel together with $5 \mu$ l PageRuler prestained protein 
ladder solution (Fermentas) for approximate the size of the proteins. The SDS gels ran for $1^{1 / 2} \mathrm{~h}$ at $90 \mathrm{~V}$. Subsequently, proteins were transferred either to Amersham HybondECL nitrocellulose membrane (GE Healthcare) or to Immun-Blot PVDF $0.2 \mu \mathrm{m}$ via dry blotting system Mini Trans-Blot Cell (BIO-RAD). For the transfer of the proteins, $220 \mathrm{~mA}$ were applied per blot for $4 \mathrm{~h}$ in a Biorad PowerPac 300 blotting apparatus. The membranes were blocked for $1 \mathrm{~h}$ at room temperature in blocking solution (TBS pH 7.4, 5\% non-fat milk powder, 0,1\% Tween 20) and then incubated overnight with the appropriate dilutions of the primary antibody in blocking solution, at $4{ }^{\circ} \mathrm{C}$. After 3 washes of 10 min each with TBST, the blots were incubated with horseradish peroxidase-conjugated secondary antibodies (diluted 1:5,000), for $1 \mathrm{~h}$ at room temperature. Protein bands were detected using the enhanced chemiluminescence (ECL) system (Pierce ECL Western Blotting Substrate, Pierce) in a NTAS ChemoCam (NTAS Science Imaging Instruments GmbH, Göttingen). For the calculation of each band intensity the ImageJ macro routine "Gel Analysis" was used. Each band of interest was manually selected and an intensity profile for every band was generated by the macro. The background intensity was calculated in the immediate vicinity of the band and was substracted from the bands intensity value. For all the samples quantified, the levels of the protein of interest in every treated condition were normalized to the levels of protein of interest in the untreated sample and were set to $100 \%$.

\subsubsection{Viral transduction of HEK 293 cell line}

The cells were plated one day prior to viral transduction in 24-well plates on poly-L-lysine coated coverslips (12 mm) as described previously (section 2.2.7) and in fresh DMEM medium supplemented only with 5\% FCS. After $24 \mathrm{~h}$ the medium was removed, the cells were washed once with pre-warmed PBS and $300 \mu \mathrm{l}$ transfection mix was added to each well. The preparation of the transfection mix was done as follows: the desired amount of virus for each well was mixed with 300 $\mu \mathrm{l}$ of DMEM and 5\% FCS inside a $1.5 \mathrm{ml}$ eppendorf tube containing a removable filter of $0.22 \mu \mathrm{m}$ pore diameter. The mix was centrifuged for $1 \mathrm{~min}$ and passed 
through the sterile filter. It was then added to the cells, kept for a maximum period of 4 days and then removed and the cells were kept for maximum another 2 days in DMEM and 5\% FCS. To optimise the viral transduction efficiency, different concentrations of each virus were used, varied between $10^{8} \mathrm{tu} / \mu \mathrm{l}-4 \times 10^{8} \mathrm{tu} / \mu \mathrm{l}$, where $\mathrm{tu} / \mu \mathrm{l}$ means transfection units per $\mu \mathrm{l}$ and corresponds to the titer unit of the virus. The efficiency was indicated by the fluorescent reporter protein that these viruses were expressing. The cells were observed every $24 \mathrm{~h}$ post transduction for their fluorescent signal and processed by immunocytochemistry when no further fluorescent difference was obvious.

\subsubsection{Embryonic transuterine otocyst injections}

This protocol has been thoroughly described in (Reuter, 2011). In this study injections were performed, using the exact same protocol by the technical assistant Christiane Senger-Freitag. Wild-type pregnant mice of CD1 genetic background at postcoidal day 11.5 were used for injections. The newborn pups were used at post-natal days 7-17 for immunohistochemistry experiments.

\subsubsection{Genotyping protocol of the pinball-wizard line}

Genomic DNA from KO and WT animals was isolated from their tales. For every sample, a small part of the tail together with $200 \mu$ l GMT buffer were placed in an eppendorf tube. For lysis, the sample was incubated at $55^{\circ} \mathrm{C}$ for $2 \mathrm{~h}$ to overnight. Proteinase $\mathrm{K}$ was heat-inactivated at $95^{\circ} \mathrm{C}$ for $10 \mathrm{~min}$ and the mixture was briefly centrifuged to seperate debris from genomic DNA in supernatant. PCR reaction was set for $2 \mu \mathrm{l}$ of every sample with $0.5 \mu \mathrm{l}$ Titanium Taq Polymerase (Invitrogen) and $10 \mathrm{x}$ of the corresponding buffer, $2.5 \mathrm{mM}$ dNTPs and $10 \mu \mathrm{M}$ of each primer. Denaturation was at $94{ }^{\circ} \mathrm{C}$ for $1 \mathrm{~min}$, primer annealing and extension at $94^{\circ} \mathrm{C}$ for $20 \mathrm{~s}, 61^{\circ} \mathrm{C}$ for $20 \mathrm{~s}$, and $68^{\circ} \mathrm{C}$ for $30 \mathrm{~s}$, repeated for 30 cycles. Final elongation was achieved at $68^{\circ} \mathrm{C}$ for 10 min. The PCR product was loaded on a $2 \%$ agarose gel and run for $1 \mathrm{~h}$ at $100 \mathrm{~V}$. 


\subsubsection{Whole-mount preparation of the organ of Corti}

Mice of postanatal day 14 (P14)-P50 were anesthetized with $\mathrm{CO}_{2}$ and killed by decapitation. The skin of the head was removed and the skull was cut in half along the midsagittal line and transferred into HEPES-buffered Hanks balanced salt solution. The brain was removed revealing the temporal bone and the labyrinth was twisted out in order to expose the cochlea. Through an opening on the apical part of the cochlea the organ of Corti was revealed and carefully the apical coil was removed away from the modiolus.

\subsubsection{RT-PCR}

PCR was performed on cDNA of adult animals (P25) Pwi. For every sample, 4 apical turns (2 animals) of Corti organs were dissected and homogenized into TRizol Reagent (Invitrogen) with a glass Teflon homogenizer. $200 \mu$ l chloroform were added and after centrifugation for $15 \mathrm{~min}$ at $13,300 \mathrm{rpm}$ at $4{ }^{\circ} \mathrm{C}$, the upper aqueous phase of RNA was isolated. To the RNA, $1 \mu$ l glycogen and $250 \mu \mathrm{l}$ isopropanol were added and was precipitated overnight at $-20^{\circ} \mathrm{C}$. Purification of RNA was achieved after another centrifugation for $45 \mathrm{~min}$ at $13,300 \mathrm{rpm}$ at $4{ }^{\circ} \mathrm{C}$ and wash with $75 \%$ ethanol. Finally, it was resuspended in $\mathrm{ddH}_{2} \mathrm{O}$.

The isolated RNA was reverse transcribed by adding $9 \mathrm{U} / \mu \mathrm{l}$ Superscript II reverse transcriptase (Invitrogen), $1.1 \mathrm{pM} / \mu \mathrm{l}$ oligo dT (Invitrogen), random hexamers (Roche), $0.45 \mathrm{nM} / \mu \mathrm{l} \mathrm{dNTPs}$ (Invitrogen), $9 \mathrm{nM} / \mu \mathrm{l}$ DTT (Invitrogen) and $1.8 \mathrm{U} / \mu \mathrm{l}$ RNaseOUT (Invitrogen) with the following protocol: 10 min room at temperature, 30 min at $37^{\circ} \mathrm{C}, 60 \mathrm{~min}$ at $42^{\circ} \mathrm{C}$ and 5 min at $70^{\circ} \mathrm{C}$. The cDNA was then used as template in PCR. The PCR was prepared in final volume $10 \mu \mathrm{l}$ with $700 \mathrm{ng}$ DNA, $5 \mu \mathrm{M}$ of forward and $5 \mu \mathrm{M}$ of reverse primer, 11.1x Buffer, and 2.5 U Platinum Taq DNA Polymerase (Invitrogen). The amplification of the transcripts was performed at 36 cycles at the following program: initial denaturation at $94^{\circ} \mathrm{C}$ for $5 \mathrm{~min}$, denaturation at $94^{\circ} \mathrm{C}$ for $1 \mathrm{~min}$, primer annealing at $57^{\circ} \mathrm{C}$ for $30 \mathrm{~s}$, and elongation 
at $72{ }^{\circ} \mathrm{C}$ for $1 \mathrm{~min}$, final elongation at $72{ }^{\circ} \mathrm{C}$ for $7 \mathrm{~min}$. The PCR products were loaded on a $1 \%$ agarose gel with the 100 bp Plus GeneRuler DNA Ladder (cat. No. \#SM0321/2/3, Fermentas). Water sample was used as negative control and as positive control, parvalbumin was amplified in all samples.

\subsubsection{Perforated patch-clamp recordings}

The electrophysiology experiments were performed on whole-mount preparation of the apical coil of the organ of Corti isolated in HEPES-buffered Hanks balanced salt solution. Each explant was transferred inside a recording chamber and fixed at the bottom with a grid of nylon threads, with the tectorial membrane facing upwards. The tissue was constantly perfused during recordings with Modified Ringers solution at a rate of at least $0.5 \mathrm{ml} / \mathrm{min}$. In order to gain access to the lateral membrane of the IHCs, the tectorial membrane, the three rows of the OHCs, the outer pillar, inner pillar and phalangeal cells were removed, using soda glass pipettes.

The patch-clamp setup was shielded by a Faraday cage, assembled on a hydraulic air table for vibration isolation (TMC, Peabody, USA) and consisted of an upright Olympus BX50WI microscope (Olympus, Hamburg, Germany) equipped with 60x/0.90 W LUMPlanFI objective. The bath level inside the recording chamber and the pipette pressure were controlled by a pump, the Ringer-Bath-Handler (Lorenz Messgerätebau GmbH, Katlenburg-Lindau, Germany). The tissue was visualised by the software Vision, version 4.0 (1996-2005 TILL Photonics GmbH, München). The pipette movement was under control of a step-motor micromanipulator (SM-5, Luigs \& Neumann, Ratingen, Germany). The electrical input/output was performed with a EPC-9 HEKA amplifier controlled by the Pulse software (HEKA, Lambrecht/Pfalz, Germany).

The perforated patch-clamp recordings were carried out essentially as described by (Sakmann and Neher, 1984). Prior to filling with the intracellular-containing amphotericin solution, the tip of the recording pipette was dipped into amphotericin-free solution, to prevent from release of the antibiotic into the extracellular environment 
of the cells. The recording pipette was made of GB150-8P borosilicate glass capillaries (Science Products, Hofheim, Germany), pulled at a Sutter P-2000 laser pipette puller (Sutter Instrument Company, Novato, USA), having a resistance of $\sim 4.5 \mathrm{M} \Omega$. It was positioned close to the IHC under constant positive pressure of $\sim 2.7 \mathrm{~cm} \mathrm{H}_{2} \mathrm{O}$ to avoid blockage of the pipette tip. After making contact with the membrane of the IHC, a gigaseal was established by application of slightly negative pressure through the recording pipette. The time needed for amphotericin to diffuse towards the tip and perforate the membrane of the IHC was estimated between 10-20 min. The recordings started when series resistance was $\sim 30 \mathrm{M} \Omega$. Membrane capacitance was measured with the Lindau-Neher technique (Lindau and Neher, 1988). $\Delta \mathrm{C}_{m}$ was estimated as the difference of the mean $\mathrm{Cm}$ over $400 \mathrm{~ms}$ after the end of the depolarization, skipping the first $80 \mathrm{~ms}$, and the mean pre-pulse Cm over $400 \mathrm{~ms}$. Currents were leak corrected with a p/4 protocol. Data analysis was performed using Igor Pro software (Wave-Metrics, Lake Oswego, OR, USA).

\subsubsection{Immunostaining}

\section{Immunohistochemistry}

The whole-mount organ of Corti was fixed in $4 \%$ formaldehyde (FA) diluted in PBS for either $1 \mathrm{~h}$ or $10 \mathrm{~min}$ on ice. Alternatively, the tissue was fixed in methanol at $-20^{\circ} \mathrm{C}$ for $20 \mathrm{~min}$. The fixative was removed by washing the tissue several times in PBS. Blocking was performed in GSDB at room temperature for $1 \mathrm{~h}$ and was followed by primary antibodies incubation diluted in GSDB at $4{ }^{\circ} \mathrm{C}$ overnight. The next day, the tissue was washed in Wash buffer and incubated with the secondary antibodies for $1 \mathrm{~h}$ at room temperature, also diluted in GSDB. Finally, the tissue was washed three times in Wash Buffer before mounted on glass slides in Mowiol 4-88 mounting medium.

\section{Immunocytochemistry}

HEK 293 cells were grown on poly-L-lysine coated coverslips. On the day of immunostaining, they were washed once with ice-cold PBS and subsequently fixed for 
20-45 min in 4\% formaldehyde (FA) in PBS. The coverslips were washed three times in PBS and incubated in the Blocking buffer for 45 min followed by overnight incubation at $4^{\circ} \mathrm{C}$ into the primary antibody, which was diluted in $5 \%$ goat serum and 0.1\% Triton X-100 in PBS. The coverslips were washed in PBS and incubated for an additional $1 \mathrm{~h}$ at room temperature with the secondary antibody. Finally, they were washed and mounted on glass slides in Mowiol 4-88 mounting medium.

\subsubsection{Confocal microscopy and image analysis}

Antibody-stained samples were imaged on a laser-scanning Leica SP2 confocal microscope using a 10x air and/or a 63x 1.4 NA oil-immersion objectives, with a blue argon $488 \mathrm{~nm}$ laser, a green HeNe (Helium Neon) $561 \mathrm{~nm}$ and a red HeNe $633 \mathrm{~nm}$ laser (Leica Mikrosysteme, Wetzlar, Germany). The vertical step size for Z-stack acquisitions was $0.5 \mu \mathrm{m}$. Acquisition was performed using the sequential line mode, so that the images from two different channels were perfectly aligned. Image analysis was performed with ImageJ and a Matlab (The Mathworks Inc., Natick, MA, USA) custom-written routine (Dr. Tina Pangršič). For the semi-quantification of otoferlin protein levels the Matlab routine calculated the fluorescent signal of maximum confocal projections of the two channels, otoferlin and VGLUT3. Polygonal regions of interest (ROI) were manually set on every individual IHC, one in apical and one in basal region of the IHC. The mean fluorescent intensity of each channel was calculated after subtracting the background fluorescence outside hair cells. The averages were calculated separately for apical and basal parts of the cells. The fluorescence data were then normalized to the fluorescence observed in IHCs from $\mathrm{Pwi}^{+/+} \mathrm{VDCR}^{+} \mathrm{CGCT}^{+}$.

\subsubsection{Electron Microscopy}

The electron microscopy was performed by Dr. Carolin Wichmann. Mouse cochleae of 21 post-natal days were explanted and fixed by perfusion for $1 \mathrm{~h}$ on ice with $4 \% \mathrm{PFA}$ and $0.5 \%$ glutaraldehyde in $1 \mathrm{x}$ PBS of $\mathrm{pH} 7.2$. Afterwards the apical turns of the organs of Corti were isolated and fixed further overnight on ice with $2 \%$ glutaraldehyde in $0.1 \mathrm{M}$ sodium cacodylate buffer of $\mathrm{pH} 7.2$. The epithelia were 
washed in sodium cacodylate buffer and placed in $1 \%$ osmium tetroxide $((\mathrm{v} / \mathrm{v})$ in 0.1 $\mathrm{M}$ cacodylate buffer) on ice for $1 \mathrm{~h}$ for further fixation, followed by $1 \mathrm{~h}$ washing step in sodium cacodylate buffer and three brief washing steps in $\mathrm{ddH}_{2} \mathrm{O}$. The epithelia were prepared as samples en bloc and stained again with $1 \%(\mathrm{v} / \mathrm{v})$ uranyl acetate in $\mathrm{ddH}_{2} \mathrm{O}$ for $1 \mathrm{~h}$ on ice. After a brief wash in $\mathrm{ddH}_{2} \mathrm{O}$, the samples were dehydrated at room temperature in increasing ethanol concentrations, infiltrated in Epon resin (100\% EtOH/Epon 1:1 (v/v), 30 and 90 min; 100\% Epon, overnight) and embedded for $48 \mathrm{~h}$ at $70^{\circ} \mathrm{C}$. The embedded samples were sliced from the anterior edge in 65 $75 \mathrm{~nm}$ sections. The sections were stained with $4 \%$ uranyl acetate and Reynold's lead citrate (Reynolds, 1963). Micrographs were obtained with a JEOL electron microscope (JEM 1011) (JEOL Germany GmbH) equipped with a Gatan Orius 1200A camera (Gatan GmbH, München, Germany) using the Digital Micrograph software package.

\subsubsection{Auditory Brainstem Responses}

The ABR recordings were performed by Nadine Hermann. The animals were anesthetized with a combination of ketamine $(125 \mathrm{mg} / \mathrm{kg})$ and xylazine $(2.5 \mathrm{mg} / \mathrm{kg})$ injected intraperitoneally. The heart rate was monitored to control for the depth of anesthesia. If necessary the anesthesia was maintained by additional injection of $0.35-0.75 \mathrm{mg}$ ketamine every 30 to $90 \mathrm{~min}$. The animal was kept at a constant temperature of $37^{\circ} \mathrm{C}$ on a heated pad. Upon the absence of the toe reflex after induction of anesthesia, three needles were inserted under the skin of the animal; the active electrode was positioned behind the ear (mastoid muscle), the reference electrode in the midline (vertex) of the head and the ground electrode near the tail. For stimulus generation and data acquisition, the TDT System III (Tucker Davis Technologies) run by BioSig32 (Tucker Davis Technologies) software was used. In order to determine the hearing thresholds of control and mutant mice, pure tones (4/6/8/12/16/24/32 kHz, $10 \mathrm{~ms}$ plateau, $1 \mathrm{~ms} \cos ^{2}$ rise/fall, intensities provided as dB SPL root mean square) were played to the anesthetized animal at a stimulus rate of $40 \mathrm{~Hz}$ and different intensities (10 dB steps) with a maximum of $90 \mathrm{~dB}$. For Click stimuli we determined the changes in amplitudes and latencies of all ABR peaks over 
the entire intensity range between threshold and $120 \mathrm{~dB}$ SPL. The speaker was calibrated with a 1/4 inch Brüel and Kjr microphone (model 4939). At and above the hearing threshold of the mice the synchronous neuronal activity of the succeeding nuclei in the auditory pathway display a characteristic series of five waves (forming a waveform) which were recorded as the 50,000 times amplified difference potential between vertex and mastoid needles (filtered at $4 \mathrm{kHz}$ low pass and $100 \mathrm{kHz}$ high pass, averaged 1,300 times). The lowest SPL generating a reproducible wave, classified by visual judgement of two independent experienced researchers, was defined as the ABR threshold.

\subsubsection{Distortion Product Otoacoustic Emissions (DPOAE)}

The function of OHCs was assessed by recording distortion product otoacoustic emissions. DPOAEs are sounds produced by the cochlea, and more specifically by the OHCs, in response to an acoustic stimulation. Thus, this test allows testing the active sound amplification process in the cochlea. The DPOAEs were usually recorded in the same animals as the ABRs. A custom-made probe containing a MKE 2 microphone (Sennheiser, USA) and an ED1/EC1 speaker system (Tucker Davis Technologies) was positioned in the ear canal. A Technics SL-P320 CD player was used to generate two primary tones $\mathrm{f} 1$ and $\mathrm{f} 2$ with ratio $\mathrm{f} 2=1.2 \mathrm{f} 1$ which were intensity-adjusted using a custom-designed mouse ear coupler and a 1/4 inch Brüel and Kjr microphone (model 4939) and sound attenuators (TDT System II). Oto-acoustic emissions were measured after playing 5 different frequency combinations, with f1 being $10 \mathrm{~dB}$ lower than f2. Every frequency combination was played for $32 \mathrm{sec}$ and tested from $10 \mathrm{~dB}$ to $60 \mathrm{~dB}$ with a step of $10 \mathrm{~dB}$. Calibration used a mouse ear coupler to predict the SPL at the ear drum. The microphone signal was digitized using a TerraTec DMX 6fire USB soundcard and Sony SoundForge software and analyzed by fast Fourier transformation using custom-written Matlab routines. 



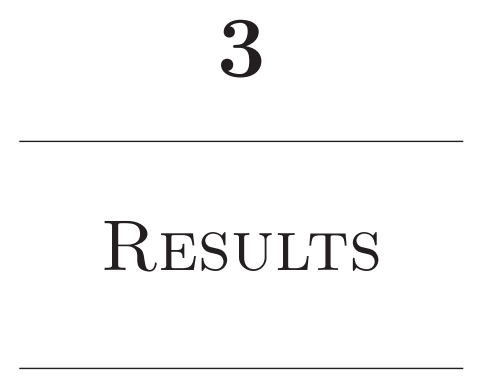

\subsection{Characterization of the Pwi mouse phenotype}

\subsubsection{The transgenic Pwi mouse line}

An insertional mutagenesis screen in zebrafish (Gross et al., 2005) revealed a variety of pathological phenotypes with defects in the development and function of the visual system. One of the identified disrupted genes was pwi, later found to be homologous to the human WRB gene (Murata et al., 2009). The human gene was initially identified to encode a protein with a tryptophan-rich carboxyl-terminal region, named WRB (Egeo et al., 1998). Fish, carrying a mutation in the wrb gene further displayed a balance and hearing (Lin et al., unpublished). This finding motivated the design of a transgenic Wrb mouse line to study the function of the WRB protein in mammals with particular focus on its role in hearing.

The $W r b$ wild-type gene contains 5 exons and the protein has three predicted transmembrane domains and one coiled-coil domain directed toward the cytosol (Vilardi et al., 2011) (see Figure 3.1 A, first row). To enable a conditional deletion of this protein, loxP sequences have been inserted between exons 1 and 5 (Figure $3.1 \mathrm{~A}$, second row). I received the conditional Wrb knockout mice ( $\mathrm{Pwi}^{\mathrm{flx} / \mathrm{flx}}$ ) (Figure 3.1 A, third line) and performed crossings with the $V D C R-C G C T$ strain (Figure 3.1 B). This mouse strain contains a transgene (VDCR) that expresses Cre under $V g$ lut3-specific promoter, and a transgene (CGCT) that expresses the enhnaced green fluorescent protein - EGFP, as Cre reporter (generated by Dr. Sonja Wojcik). Since I was particularly interested in the effects of the deletion of Wrb at the IHC synapse, the VDCR-CGCT line was chosen to specifically activate Cre recombinase in cells 
A

Wrb gene

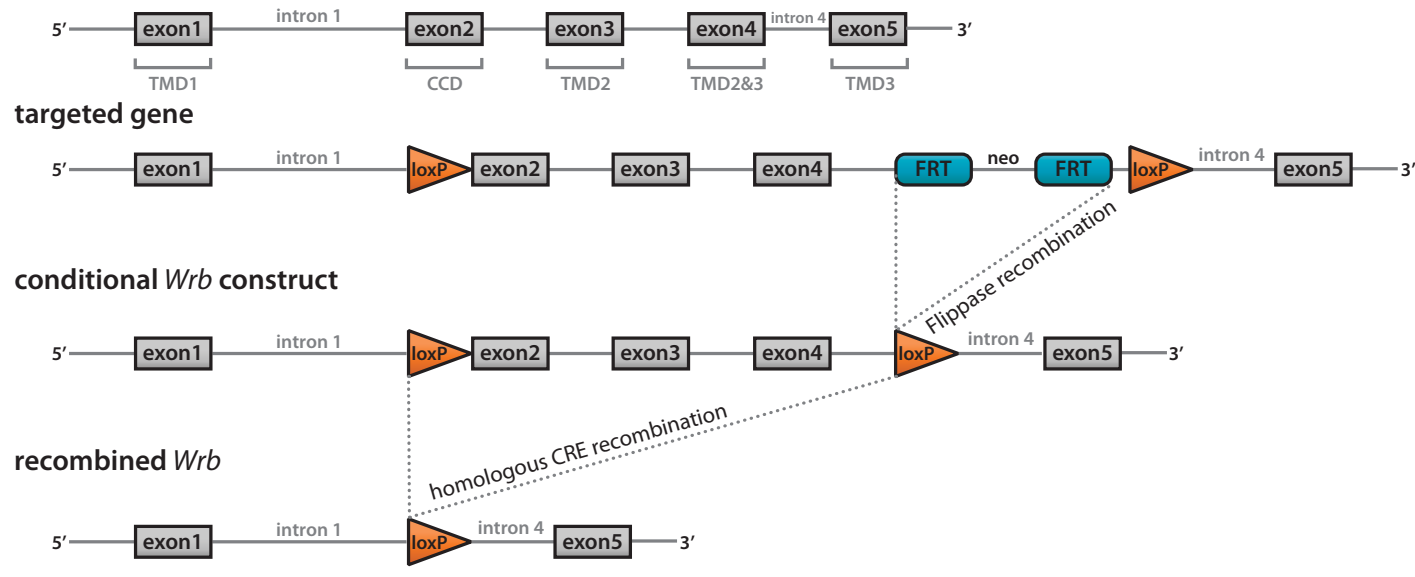

B

VDCR transgene

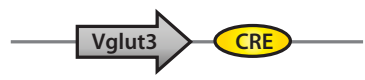

CGCT transgene

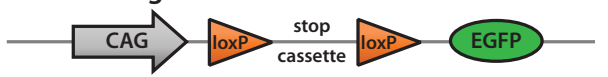

C
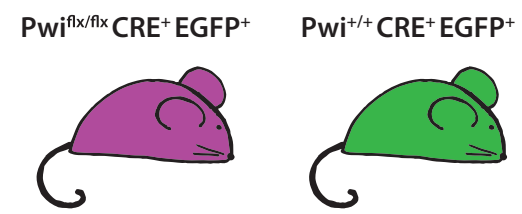

Pwi ${ }^{\text {if/ffix }}$ CRE- EGFP +1

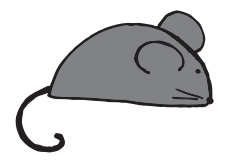

Figure 3.1: Targeted disruption of the $\boldsymbol{W r b}$ gene locus. (A) Maps of the wild-type Wrb genomic locus, the targeted $W r b$ gene, the conditional Wrb floxed construct after Flippase homologous recombination and the predicted structure of the recombined Wrb locus after homologous Cre recombination and excision of exons 2-4. The diagram shows (from top to bottom) the targeting strategy followed for generation of the conditional pinball-wizard KO mouse line. Initially, the wild-type $W r b$ genomic locus was targeted by the FRT/loxP recombination system. Insertion of a neomycin (neo) cassette, flanked by Flp (flippase) Recombination Target (FRT) sites, served as selection marker of embryonic stem cells to be used for injection into blastocyst of pregnant mouse. The offspring with the targeted gene were crossed with a flippase-expressing mouse line to remove the neo cassette by FRT site-directed recombination. (B) The two transgenes of the VGlut3-CRE specific EGFP reporter line VDCR-CGCT. Cre-directed homologous recombination at loxP sites of the conditional $\mathrm{Wrb}$ floxed construct and removal of the stop cassette before EGFP gene enables $W r b$ deletion with concomitant EGFP expression in tissues expressing VGLUT3. (C) The three mouse genotypes used in this study, $\mathrm{Pwi}^{\mathrm{fx} / \mathrm{flx}} \mathrm{VDCR}^{+} \mathrm{CGCT}^{+}$(KO) (in violet), control $\mathrm{Pwi}^{+/+} \mathrm{VDCR}^{+} \mathrm{CGCT}^{+}$(in green) and control $\mathrm{Pwi}^{\mathrm{fx} / \mathrm{flx}} \mathrm{VDCR}^{-} \mathrm{CGCT}^{+/-}$(in gray), referred as $\mathrm{Pwi}^{\mathrm{flx} / \mathrm{flx}} \mathrm{VDCR}^{-} \mathrm{CGCT}^{+}$in the rest of the results. 
expressing VGLUT3. In addition to the cells of interest (IHCs), VGLUT3 is further expressed in the brain in glutamatergic, cholinergic, serotoninergic, and GABAergic synapses (Fremeau et al., 2001). Additionally, VGLUT3 can be found in retina, heart, liver, and kidney (Munguba et al., 2011), thus exposing these tissues to Cre recombinase activity and potential defects caused by the conditional knockout (KO) of the WRB protein in our mouse line. The resulting genotypes of the new Wrb mutant mice (Pwi further on) are shown in Figure 3.1 C. In the $\mathrm{Pwi}^{\mathrm{Ax} / \mathrm{flx}} \mathrm{VDCR}^{+}$ $\mathrm{CGCT}^{+}(\mathrm{KO})$ mice homologous recombination disrupts the Wrb gene, which is being reported by EGFP positive IHCs. Throughout this thesis the results obtained in the $\mathrm{KO}$ animals are represented in violet colour. $\mathrm{Pwi}^{+/+} \mathrm{VDCR}^{+} \mathrm{CGCT}^{+}$mice serve as one control group and are symbolized by green colour. Pwi ${ }^{\mathrm{flx} / \mathrm{flx}} \mathrm{VDCR}^{-}$ $\mathrm{CGCT}^{+}$and $\mathrm{Pwi}^{\mathrm{fx} / \mathrm{flx}} \mathrm{VDCR}^{-} \mathrm{CGCT}^{-}$mice (from now on for simplification combined and together denoted as $\mathrm{Pwi}^{\mathrm{flx} / \mathrm{fx}} \mathrm{VDCR}^{-} \mathrm{CGCT}^{+}$) are considered as a second control group, since in the absence of Cre recombinase neither the Wrb construct nor the CGCT can be recombined. The results obtained in these mice are shown in gray/black colour (Figure 3.1 C). The conditional Wrb construct should not disrupt the genomic locus of $W r b$ and is not expected to cause any deletion of the protein when not recombined.

\subsubsection{The conditional Pwi knockout exhibits seizure-like events}

The conditional Pwi KO mice were smaller in size than both control littermates and frequently trembled and showed a tendency to lean toward the side. In many cases, the $\mathrm{KO}$ adopted a crooked body posture and hit its head against the cage walls. The KO animals typically died within 2 months after birth. The most striking aspect of the obvious behavioral phenotype of the $\mathrm{KO}$ were episodes of neurological attacks resembling epileptic seizures. The animals were suddenly stiffened up, loosing balance and probably consciousness and then, they were beginning to jerk, with rapid body movements and high tension in the body muscles. Whenever possible, videos of such events were recorded and examples of the animal's struggling can be seen in Figure 3.2. Attacks were initiated without any known reason and lasted for several minutes. 

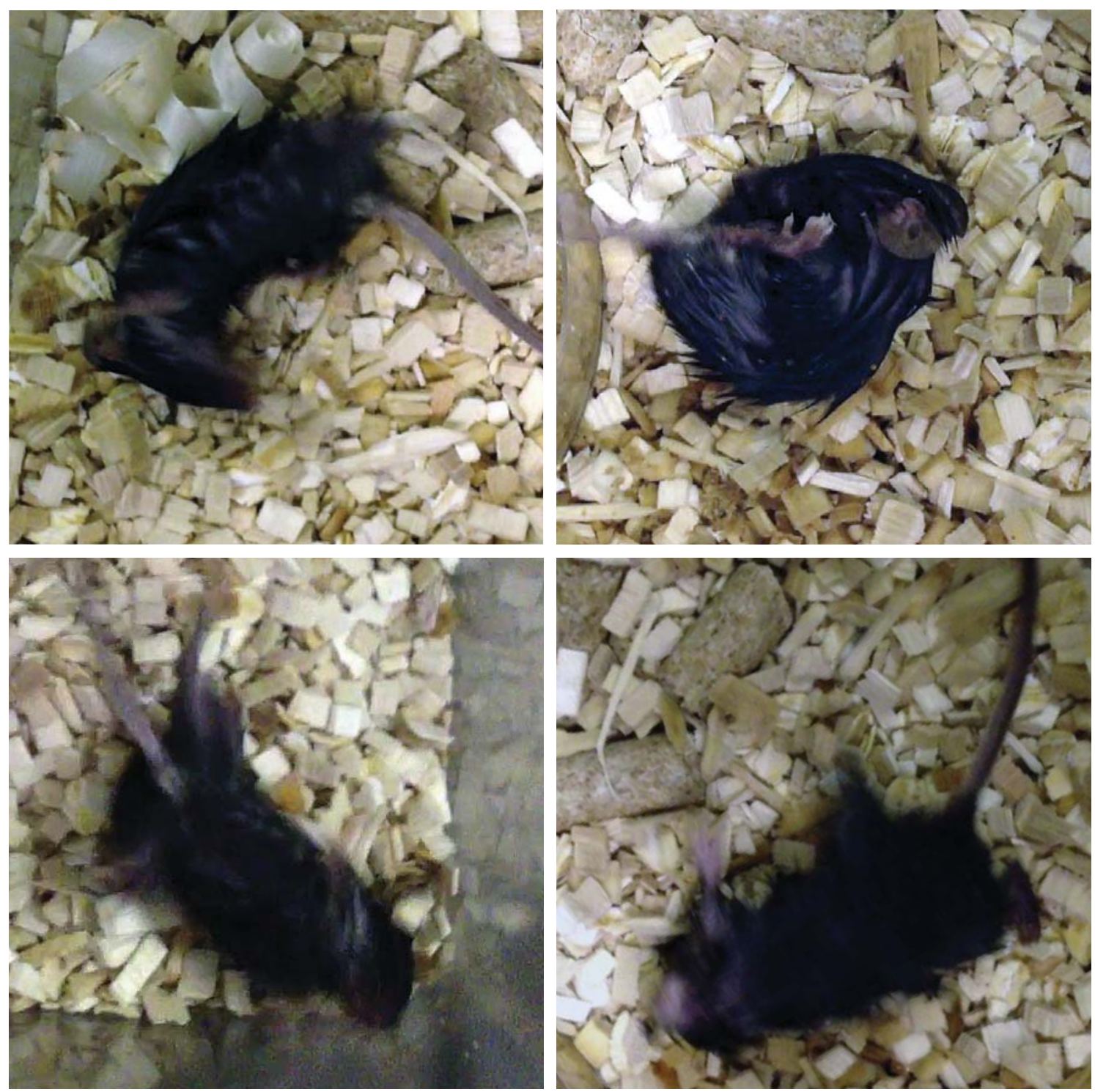

Figure 3.2: The behavior of the conditional Pwi KO mice. Snapshots from video recorded during a presumable epileptic episode of a $\mathrm{Pwi}^{\mathrm{flx} / \mathrm{flx}} \mathrm{VDCR}^{+} \mathrm{CGCT}^{+}$animal.

Animals showing this behavior were sacrificed to avoid further suffering. Whether every KO animal experiences at least once epileptic seizure is not known. If epileptic attacks are the cause of death is also not yet clear. None of the two controls was ever reported to show a similar behavior. They also had a normal life expectancy. 


\subsubsection{Deletion of $W r b$ causes a progressive hearing defect}

Since hearing is impaired in pwi zebrafish mutant (Lin et al., unpublished), I asked whether the absence of WRB protein also affects hearing of the conditional Pwi KO mouse and whether it does so in an age-dependent manner. To answer this question, I selected three age groups and the audiologist Nadine Hermann performed auditory brainstem response $(\mathrm{ABR})$ recordings. The age groups were selected according to the following criteria: the first group (group I) included juvenile mice from P14-P19, which developmentally follows the onset of hearing in mice (P12) (Mikaelian and Ruben, 1965). Therefore, this is an interesting time-point, since the auditory system is still under maturation. An additional advantage of choosing this age group is that it allows for direct comparison to the electrophysiology data, which will be described in a later section (3.1.6). The second group (group II) consisted of 3- to 4-week old mice (P21-P29), which, according to our knowledge on wild-type (WT) C57Bl/6J mice, have a mature auditory function. The last group (group III) consisting of animals older than 30 days (>P30) was selected to test for possible further deterioration of hearing in the $\mathrm{KO}$ animals shortly before their death. Animals from all three genotypes $\left(\mathrm{Pwi}^{\mathrm{flx} / \mathrm{flx}} \mathrm{VDCR}^{+} \mathrm{CGCT}^{+}, \mathrm{Pwi}^{\mathrm{flx} / \mathrm{flx}} \mathrm{VDCR}^{-} \mathrm{CGCT}^{+}\right.$, and $\mathrm{Pwi}^{+/+}$ $\mathrm{VDCR}^{+} \mathrm{CGCT}^{+}$) in all age groups were tested for the auditory system function. ABR recordings provide information about the response of the auditory pathway to various stimuli and provide estimates of hearing threshold. Sound stimuli elicit characteristic waveforms of the difference potential between mastoid and vertex, as can be seen in Figure 3.3 A. The two control groups showed normal ABR waveforms in response to the $80 \mathrm{~dB}$ SPL click stimulus with comparable amplitude and latency at all 3 selected ages. However, in the $\mathrm{KO}$ mice, a response to sound was observed only in the youngest age group (group I), where however wave I could not be elicited and wave III showed a reduced amplitude. At later ages (group II and III) no ABR activity could be detected.

Another interesting observation that can be made from the ABR waveform graphs is the occurrence of a low peak or a hump just before wave I, which represents the summating potential. The summating potential, or receptor potential, is derived from the combined electrical response of IHCs and OHCs to sound stimulation and 


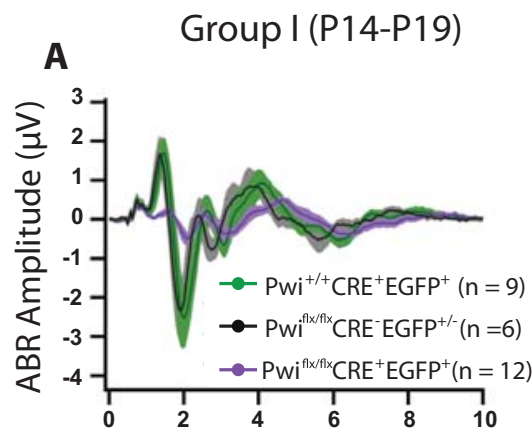

Group II (P21-P29)

Group III (>P30)
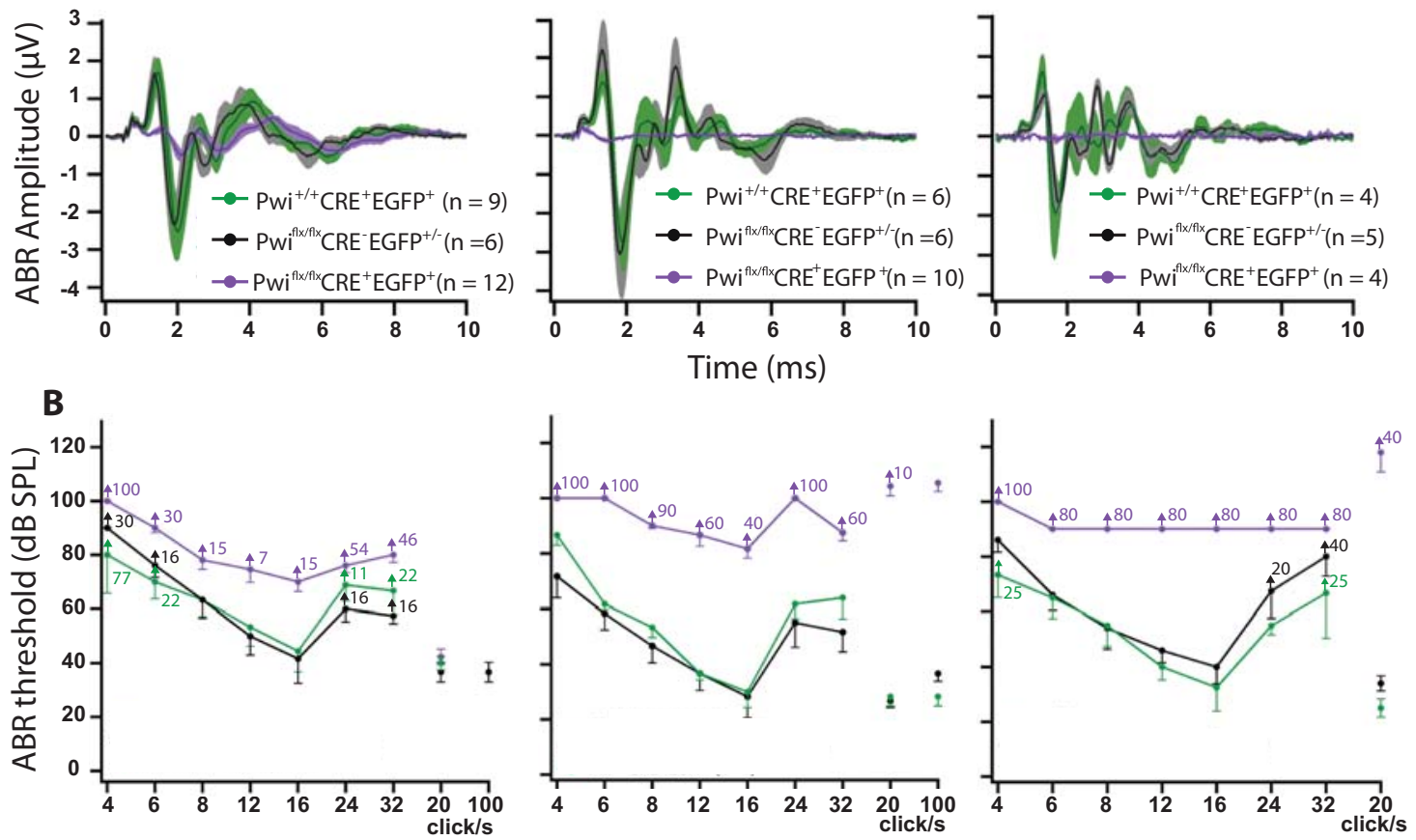

Frequency $(\mathrm{kHz})$

Figure 3.3: Assessment of hearing in Pwi mouse line. (A) Averaged ABR waveforms of each genotype in response to $80 \mathrm{~dB}$ SPL click stimuli. In goup II and III no ABR responses can be evoked in $\mathrm{Pwi}^{\mathrm{fl} / \mathrm{flx}} \mathrm{VDCR}^{+} \mathrm{CGCT}^{+}$animals. Note a noticeable hump at the beginning of the response in the $\mathrm{ABR}$ trace of the $\mathrm{Pwi}^{\mathrm{flx} / \mathrm{flx}} \mathrm{VDCR}^{+} \mathrm{CGCT}^{+}$animals of group I and II that likely represents the summating potential of hair cells and disappears in the age group III. (B) ABR audiograms with the average hearing thresholds for every genotype. Arrows with numbers show the percentage of animals in which hearing threshold could not be reached within the range of our recording equipment (up to $90 \mathrm{~dB} \mathrm{SPL}$ ). Data in A and B are presented for three different age groups, $\mathrm{n}$ shows the number of animals recorded.

indicates the presence of at least partially functional mechanotransduction in hair cells. The KO mice exhibit a summating potential mostly in group I, less in group II, and not at all in group III. The average hearing thresholds (mean \pm s.e.m.) for all age groups are presented in Figure 3.3 B, with arrows indicating animals with immeasurable hearing thresholds (> $90 \mathrm{~dB}$ SPL). Technical limitations of the recording system did not allow for detection of responses elicited with sounds of intensities higher than $90 \mathrm{~dB}$ and so an arbitrary value (100 dB SPL) was assigned. 
A

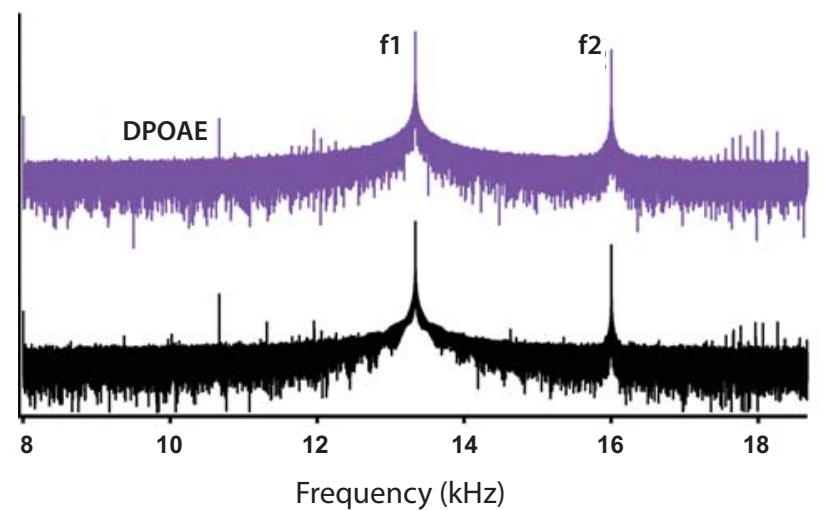

B

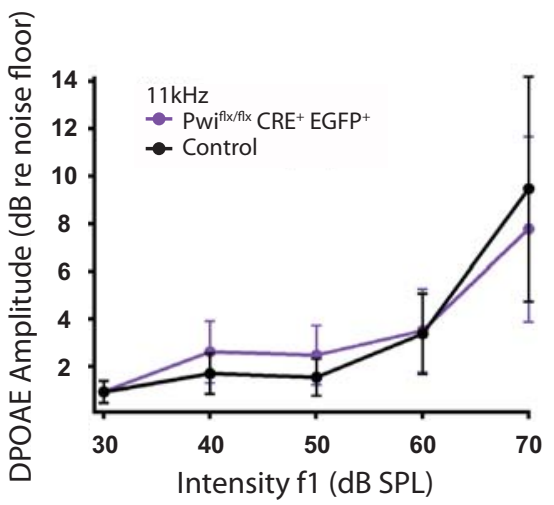

Figure 3.4: Assessment of Hearing-DPOAE. (A) Representative power spectrum of the microphone signal with the two primary tones (f1 and $\mathrm{f} 2$ at $60 \mathrm{~dB}$ SPL) played at the ear canal of the mouse during distortion product otoacoustic emission (DPOAE) recordings. (B) DPOAE amplitudes in mice of the age group II. As controls, $\mathrm{Pwi}^{+/+} \mathrm{VDCR}^{+} \mathrm{CGCT}^{+}$and $\mathrm{Pwi}^{\mathrm{flx} / \mathrm{flx}} \mathrm{VDCR}^{-}$ $\mathrm{CGCT}^{+}$animals were taken $\left(\mathrm{Pwi}^{\mathrm{flx} / \mathrm{flx}} \mathrm{VDCR}^{+} \mathrm{CGCT}^{+} \mathrm{n}=5\right.$, controls $\left.\mathrm{n}=5\right)$.

The arrows symbolize this and the values next to them represent the percentage of animals that showed no detectable thresholds. The hearing thresholds in the two control groups were comparable, showing a normal hearing, whereas in the KO animals an age-dependent hearing loss was evident. The age group I animals responded with high thresholds to tone bursts, but displayed normal responses to click stimuli. Older KO mice on the other hand (group II and III), hardly responded to tone burst sounds and clicks. The results presented so far demonstrate a hearing impairment in the Pwi KO, which becomes profound in adult animals, with ABRs being hardly (group II, Figure 3.3 A, middle graph) or not at all (group III, Figure 3.3 A, right graph) elicited by tone bursts or clicks.

Additionally, distortion product otoacoustic emissions (DPOAE) were recorded mostly in animals from age group II. Here, the age group I was not tested, as in young animals the ear canal was very narrow and the microphone could not be positioned properly. Because of the small number of animals recorded for DPOAE, the control group includes data from both $\mathrm{Pwi}^{\mathrm{flx} / \mathrm{flx}} \mathrm{VDCR}^{-} \mathrm{CGCT}^{+}$and $\mathrm{Pwi}^{+/+} \mathrm{VDCR}^{+}$ $\mathrm{CGCT}^{+}$genotypes. As the data suggest (Figure $3.4 \mathrm{~B}$ ), KO animals behave simi- 
larly to the controls and produce normal DPOAEs, in response to increasing sound intensities $(20,40,60,80 \mathrm{~dB}$ SPL). Taking together, this result and the presence of a summating potential indicate the presence of functional sound amplification in the cochlea and mechanotransduction by the OHCs in the $\mathrm{KO}$ mice, at least at young age.

\subsubsection{The morphology of the Pwi organ of Corti}

Cre-mediated recombination and likely cell-specific excision of the Wrb gene in KO mice and correct genotyping for $V D C R-C G C T$ in all groups, were verified by the expression of the EGFP reporter in IHCs. Characteristic examples of the 3 different genotypes used in this study, are presented in Figure 3.5. The fluorescent signal in the KO ( $\left.\mathrm{Pwi}^{\mathrm{fx} / \mathrm{fx}} \mathrm{VDCR}^{+} \mathrm{CGCT}^{+}\right)$IHCs indicated the recombination of the Wrb gene and served as guide during the cell physiology experiments. Organs of Corti of all genotypes were found to be normal with no gross morphological changes in light microscopy (Figure 3.5, lower panel). Green fluorescence was not only found in IHCs, but often, also in OHCs (Figure 3.5, upper panel, middle image), indicating that the Vglut3 promoter is also active in the OHCs. The fluorescent signal was detected in $\sim 80 \%$ of the IHCs in both the apical and basal (not shown here) parts of the organs of Corti of $\mathrm{CRE}^{+} \mathrm{EGFP}^{+}$mice.

\subsubsection{The conditional $W r b$ construct is recombined only in the presence of Cre recombinase}

As an additional way to confirm the recombination of the conditional Wrb construct, I checked all three selected pinball-wizard genotypes by means of RT-PCR. Since the loxP sites presumably surround only exons 2-4 in the targeted construct, I could design primers that would allow me to test the length of the transcript to determine whether it was recombined or not. The first loxP should be inserted inside intron 1 , just upstream of exon 2, while the second loxP is located in intron 4. By designing primers that would bind inside exon 1 (forward primer) and at the beginning of exon 2 (reverse primer), I was expecting to see $W r b$ transcripts of equal length in the $\mathrm{Pwi}^{+/+} \mathrm{VDCR}^{+} \mathrm{CGCT}^{+}$and $\mathrm{Pwi}^{\mathrm{fx} / \mathrm{flx}} \mathrm{VDCR}^{-} \mathrm{CGCT}^{+}$organs of Corti, but no 
transcript in the $\mathrm{Pwi}^{\mathrm{flx} / \mathrm{flx}} \mathrm{VDCR}^{+} \mathrm{CGCT}^{+}$.

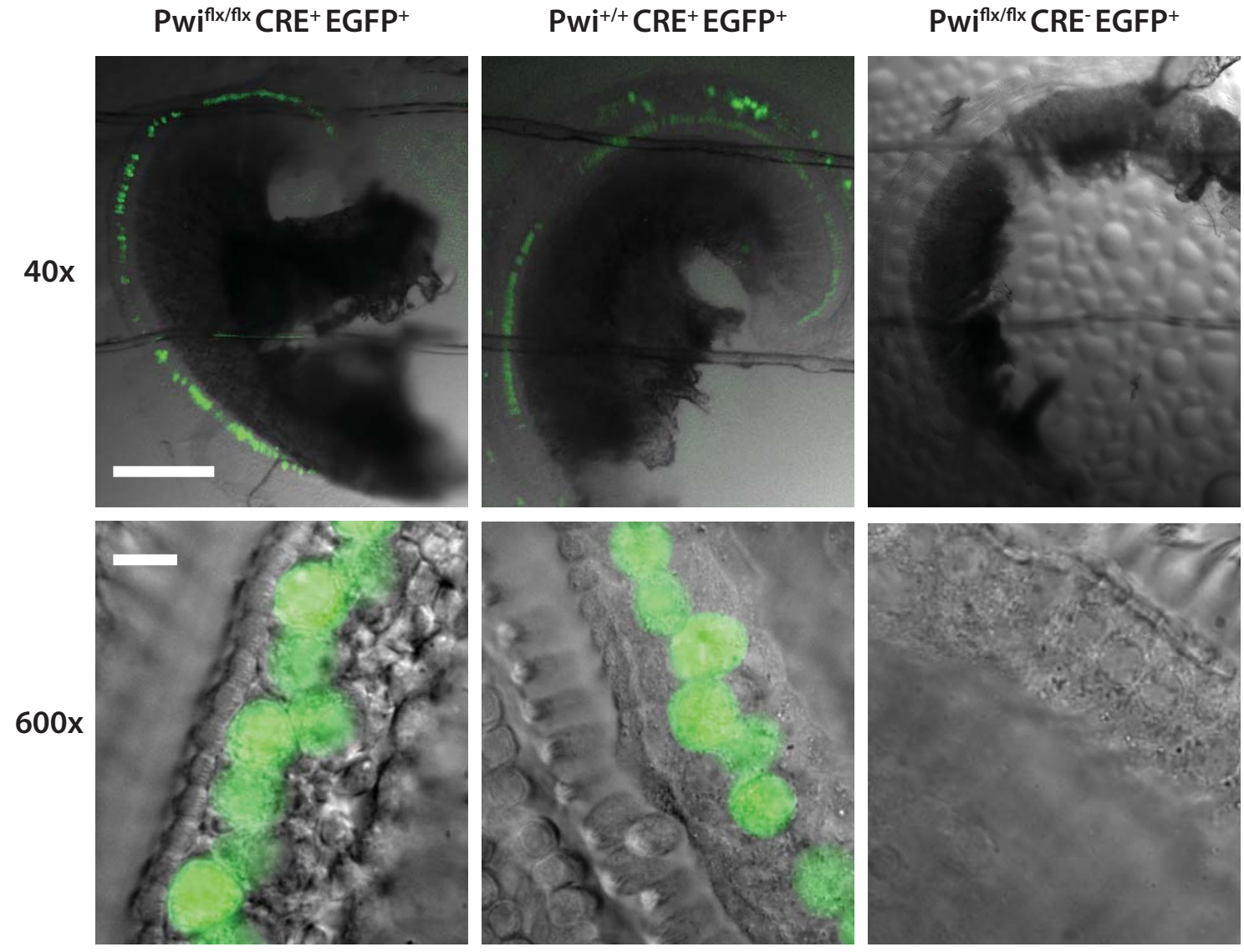

Figure 3.5: Cre activity is indicated by green fluorescent IHCs. Representative images (snapshots) of apical coils of organs of Corti at 40x and 600x magnification. Note green fluorescent cells in $\mathrm{CRE}^{+}$but not in $\mathrm{CRE}^{-}$organs of Corti, which represent activation of Cre-mediated recombination and consequent expression of EGFP. In organs of Corti, green fluorescence is mostly observed in IHCs. Scale bar at 40x magnification: $0.2 \mathrm{~mm}$, scale bar at 600x magnification: 10 $\mu \mathrm{m}$.

Interestingly, as shown in Figure 3.6 A, this was not the case. If the first loxP is indeed located inside intron 2 , the exon 2 should be lost upon recombination in the $\mathrm{KO}$ and the reverse primer should not be able to find a binding substrate. On the contrary, primers designed to bind to exons 1 (forward) and 5 (reverse) indicated that recombination in $\mathrm{KO}$ had taken place. These primers could generate a long transcript when recombination did not occur and exons $2-4$ were present (as is the case for $\mathrm{Pwi}^{+/+} \mathrm{VDCR}^{+} \mathrm{CGCT}^{+}$and $\mathrm{Pwi}^{\mathrm{flx} / \mathrm{flx}} \mathrm{VDCR}^{-} \mathrm{CGCT}^{+}$), but generate a 
smaller transcript when the exons 2-4 were excised (Figure 3.6 B). Organs of Corti

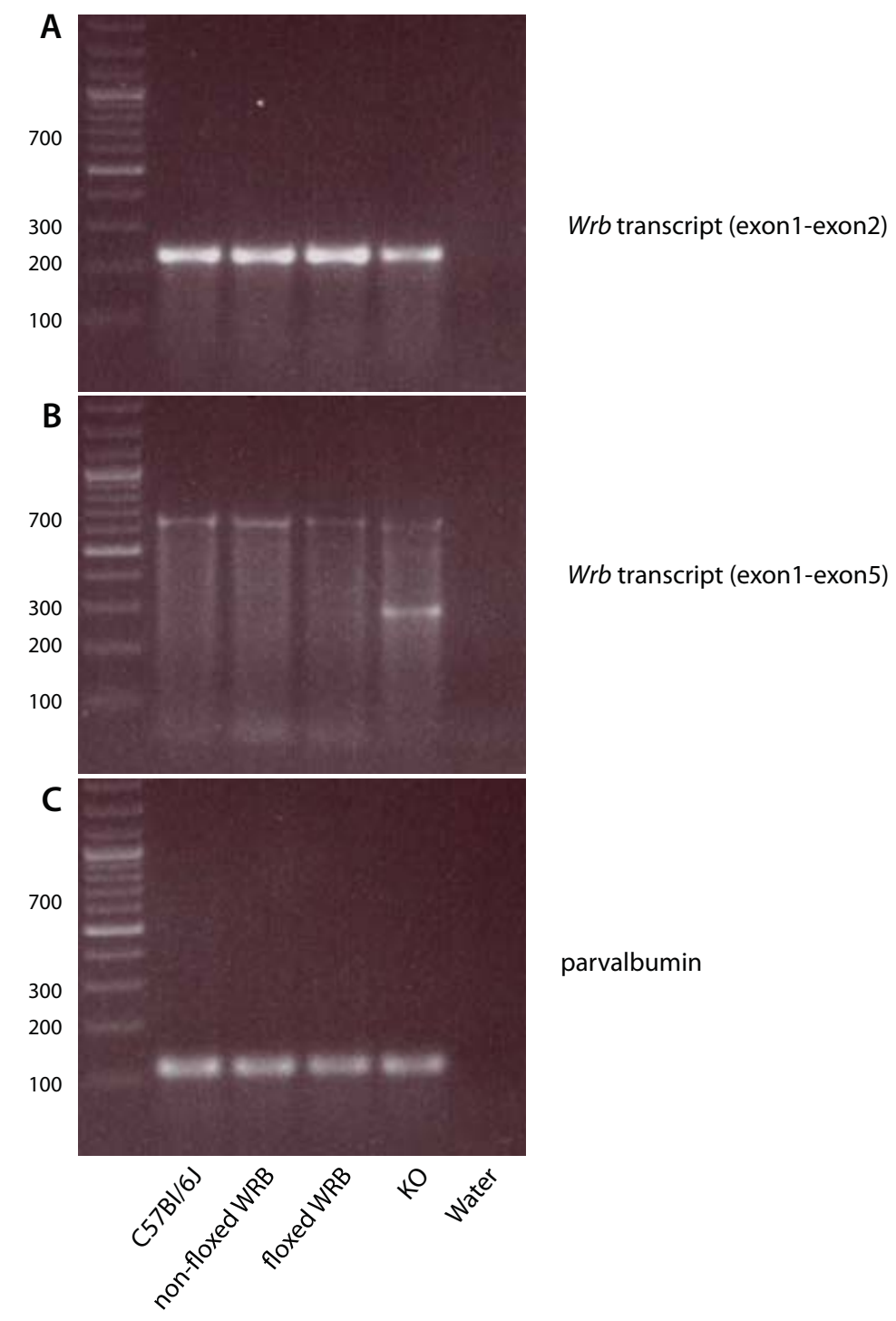

Figure 3.6: Testing the recombination by RT-PCR. Amplification of the transcripts spanning (A) exon 1 to 2 and (B) exon 1 to 5. (C) Amplification of parvalbumin as positive control. For simplicity KO denotes $\mathrm{Pwi}^{\mathrm{fx} / \mathrm{flx}} \mathrm{VDCR}^{+} \mathrm{CGCT}^{+}$, floxed $\mathrm{WRB}$ denotes $\mathrm{Pwi}^{\mathrm{fl} / \mathrm{flx}} \mathrm{VDCR}^{-}$ $\mathrm{CGCT}^{+/-}$, and non-floxed WRB denotes $\mathrm{Pwi}^{+/+} \mathrm{VDCR}^{+} \mathrm{CGCT}^{+}$animals.

from C57Bl/6J mice were used as external control and a sample containing water and no DNA was used to detect potential contamination. Primers against parvalbumin, an abundant IHC cytosolic protein that binds $\mathrm{Ca}^{2+}$, were used as a positive control reaction to test the quality of the samples (Figure 3.6 C). It should be noted 
that the PCR was performed on whole organs of Corti (4 from each genotype) and not on single cells. Thus, in the case of the $\mathrm{Pwi}^{\mathrm{flx} / \mathrm{flx}} \mathrm{VDCR}^{+} \mathrm{CGCT}^{+}$, $\mathrm{IHCs}_{\text {shat }}$ were negative for Cre activity as well as other cell types, carrying the Wrb gene were also included and generated a product for non-recombined transcript (Figure 3.6 B, at the $\mathrm{KO}$ sample the faint band at $~ 700 \mathrm{bp}$.)

\subsubsection{Disruption of synaptic function in $\mathrm{Pwi}^{\mathrm{flx} / \mathrm{flx}} \mathrm{VDCR}^{+} \mathrm{CGCT}^{+}$and $\mathrm{Pwi}^{\mathrm{flx} / \mathrm{flx}} \mathrm{VDCR}^{-} \mathrm{CGCT}^{+}$IHCs}

After the observation of a progressive hearing loss in $\mathrm{KO}$ animals, I was intrigued to investigate the mechanism of deafness at the level of the IHCs. I chose perforated patch-clamp measurements of $\mathrm{Ca}^{2+}$ currents and membrane capacitance to test the presynaptic function in apical IHCs of $\mathrm{Pwi}^{\mathrm{flx} / \mathrm{flx}} \mathrm{VDCR}^{+} \mathrm{CGCT}^{+}, \mathrm{Pwi}^{\mathrm{flx} / \mathrm{flx}}$ $\mathrm{VDCR}^{-} \mathrm{CGCT}^{+}$, and $\mathrm{Pwi}^{+/+} \mathrm{VDCR}^{+} \mathrm{CGCT}^{+}$mice at P14-P17 (age group I). The $\mathrm{Ca}^{2+}$ currents in the IHCs of the 3 genotypes were similar in amplitude (Figure 3.7 A), indicating an intact $\mathrm{Ca}^{2+}$ signaling. Characteristic examples of $\mathrm{Ca}^{2+}$ current kinetics and capacitance changes in response to a $100 \mathrm{~ms}$ depolarization duration stimulus are shown in Figure $3.7 \mathrm{~B}$ and $\mathrm{C}$, respectively. The process of exocytosis can be monitored by membrane capacitance measurements. Membrane capacitance reflects the surface area of the membrane and changes in membrane capacitance are proportional to increases or decreases in cell's surface area, due to exocytosis or endocytosis, which often do not overlap in their timing and hence can be reported separately by the same measurement over time. To evoke exocytosis, cells were depolarized to the voltage where maximal currents were observed (Figure $3.7 \mathrm{~A}$ ) for various durations. The observed changes in membrane capacitance and calcium charge are displayed in Figure 3.7 D. The results demonstrate a significant difference between exocytic responses in $\mathrm{Pwi}^{\mathrm{flx} / \mathrm{flx}} \mathrm{VDCR}^{+} \mathrm{CGCT}^{+}$and $\mathrm{Pwi}^{+/+} \mathrm{VDCR}^{+}$ $\mathrm{CGCT}^{+}$IHCs and unexpectedly showed perturbed exocytosis also in one of the control genotypes, the $\mathrm{Pwi}^{\mathrm{flx} / \mathrm{flx}} \mathrm{VDCR}^{-} \mathrm{CGCT}^{+}$. From the graphs displaying $\Delta \mathrm{C}_{m}$ as a function of the depolarization duration (Figure 3.7 D, upper graph), I could extract information about the amplitude and kinetics of the release of the readily releasable pool (RRP) and the sustained component of exocytosis for all genotypes. 
A
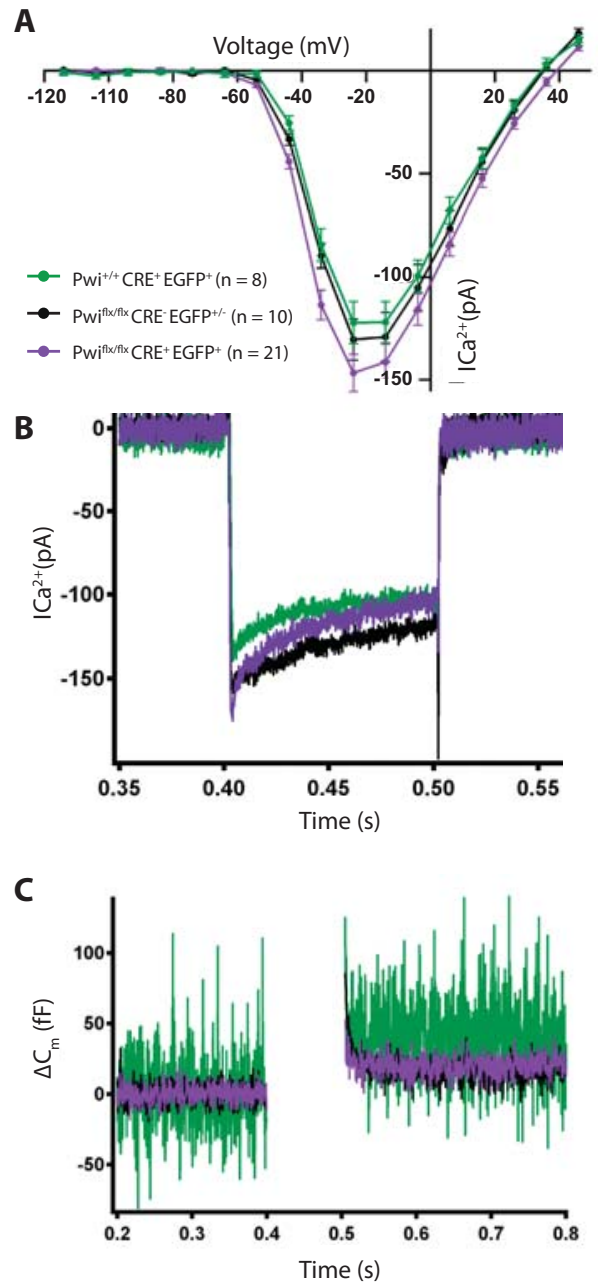
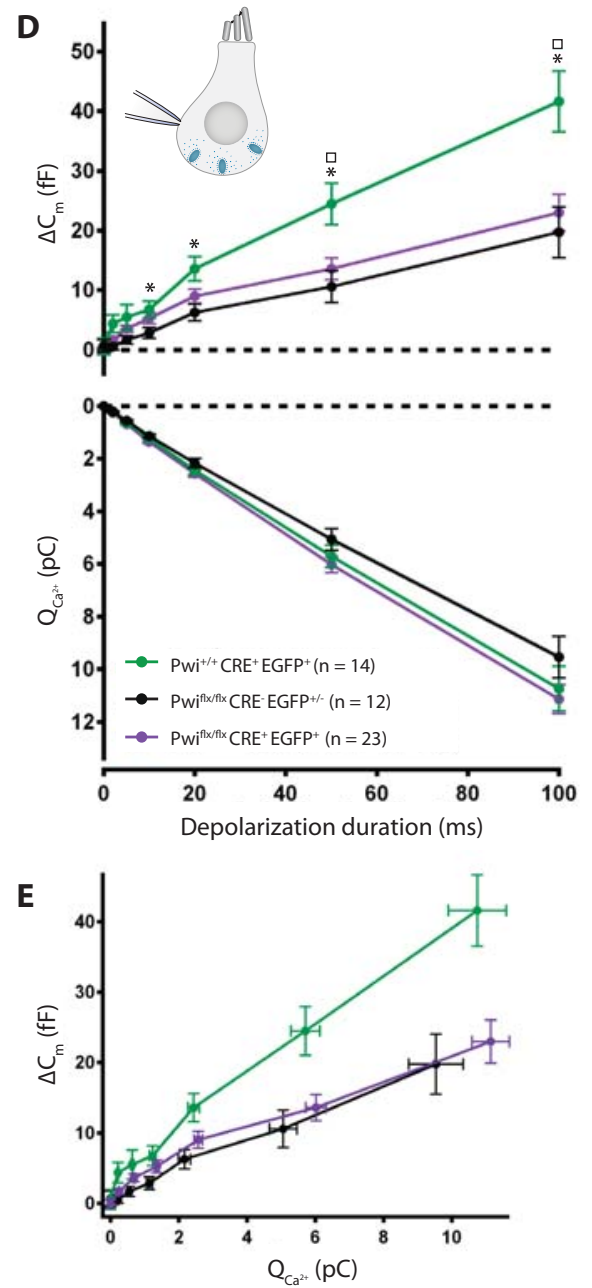

Figure 3.7: IHC synaptic function of the Pwi mouse line. (A) Current-voltage (I-V) relationship in IHCs of all three genotypes in $2 \mathrm{mM}$ extracellular $\left[\mathrm{Ca}^{2+}\right]$. (B) Representative $\mathrm{Ca}^{2+}$ currents and (C) $\mathrm{C}_{m}$ changes in IHCs in response to $100 \mathrm{~ms}$ depolarizations. (D) Kinetics of exocytosis, $\Delta \mathrm{C}_{m}$, (top) and corresponding $\mathrm{Ca}^{2+}$ current integrals, $\mathrm{QCa}^{2+}$ (bottom) at various depolarization durations. (E) $\Delta \mathrm{C}_{m}$ plotted versus $\mathrm{QCa}^{2+}$. All measurements were performed in IHCs from age group I (P14-P17). $\mathrm{n}$ shows the number of cells recorded. Data points represent mean values \pm s.e.m. All data were tested for normality with Jarque-Bera and Kolmogorov-Smirnov tests and for equal variances. Unless otherwise stated, a Mann-Whitney-Wilcoxon test was employed (non Gaussian distributions and unequal variances.) $\square$ and $\star$ denote the significant difference between $\mathrm{Pwi}^{\mathrm{fx} / \mathrm{flx}} \mathrm{VDCR}^{+} \mathrm{CGCT}^{+}$(violet) and $\mathrm{Pwi}^{+/+} \mathrm{VDCR}^{+} \mathrm{CGCT}^{+}$(green), $\mathrm{Pwi}^{+/+} \mathrm{VDCR}^{+}$ $\mathrm{CGCT}^{+}$and $\mathrm{Pwi}^{\mathrm{fx} / \mathrm{flx}}$ VDCR- $\mathrm{CGCT}^{+}$(black), respectively.

Although, in general, the exocytic release of the $\mathrm{Pwi}^{\text {flx } / \mathrm{flx}} \mathrm{VDCR}^{+} \mathrm{CGCT}^{+} \mathrm{IHCs}$ 
was reduced as compared to the control Pwi ${ }^{+/}+\mathrm{VDCR}^{+} \mathrm{CGCT}^{+} \mathrm{IHCs}$, this difference only became significant for the sustained release $(\mathrm{p}=0.007$ for $50 \mathrm{~ms}$ and $\mathrm{p}=0.002$ for $100 \mathrm{~ms})$. At shorter depolarization durations $(5-20 \mathrm{~ms})$, by probing the release of the RRP, no significant difference was detected. Interestingly, in control $\mathrm{Pwi}^{\mathrm{flx} / \mathrm{flx}} \mathrm{VDCR}^{-} \mathrm{CGCT}^{+} \mathrm{IHCs}$, the $\mathrm{C}_{m}$ responses at almost all stimulus durations were significantly decreased compared to the other control group, Pwi $\mathrm{i}^{+/+}$ $\mathrm{VDCR}^{+} \mathrm{CGCT}^{+}(\mathrm{p}=0.01$ for $10 \mathrm{~ms}, \mathrm{p}=0.007$ for $20 \mathrm{~ms}, \mathrm{p}=0.005$ for $50 \mathrm{~ms}$, and $\mathrm{p}=0.002$ for $100 \mathrm{~ms}$ ). Since calcium currents seemed to be reduced in $\mathrm{Pwi}^{\mathrm{fx} / \mathrm{flx}}$ $\mathrm{VDCR}^{-} \mathrm{CGCT}^{+}$IHCs, membrane capacitance responses versus $\mathrm{Ca}^{2+}$ charge were plotted (Figure $3.7 \mathrm{E}$ ), which demonstrate a match in the efficiency of exocytosis in $\mathrm{Pwi}^{\mathrm{flx} / \mathrm{flx}} \mathrm{VDCR}^{+} \mathrm{CGCT}^{+}$and $\mathrm{Pwi}^{\mathrm{flx} / \mathrm{flx}} \mathrm{VDCR}^{-} \mathrm{CGCT}^{+}$IHCs. Together, the electrophysiological data report that the IHCs of the KO Pwi are able to establish a normal RRP, but exhibit diminished sustained exocytosis, which likely affects hearing. However, exocytosis was comparably reduced in IHCs of the control Pwi $\mathrm{P}^{\mathrm{fx}} / \mathrm{flx}$ $\mathrm{VDCR}^{-} \mathrm{CGCT}^{+}$, which showed normal hearing as assayed by ABR, a discrepancy that remains unsolved.

\subsubsection{Slowed vesicle pool replenishment in IHCs of $\mathrm{Pwi}^{\mathrm{flx} / \mathrm{flx}} \mathrm{VDCR}^{+}$ $\mathrm{CGCT}^{+}$mice}

The data acquired from studying membrane capacitance were further used to calculate different kinetic parameters of the exocytosis of the $\mathrm{KO}$ and control Pwi IHCs. I fitted the mean exocytic $\Delta \mathrm{C}_{m}$ values (Figure $3.7 \mathrm{D}$, upper graph), as a function of stimulus durations of every genotype using the sum of an exponential and a linear function. By these means, I estimated the size of the RRP of each genotype by dividing the amplitude of the exponential function by the synapse number per IHC (see section 3.2.1, Table 2) and by the single vesicle capacitance ( $\sim 45 \mathrm{aF}$ ) (Neef et al., 2007). Additionally, the slope of the linear function provided information for the rate of the sustained exocytosis. Sustained exocytosis was calculated by dividing the slope of the linear function by the synapse number and by the single vesicle capacitance (as described in Pangršič et al., 2010) (Figure 3.8 Table 1). Inner hair cells of the $\mathrm{Pwi}^{\mathrm{flx} / \mathrm{flx}} \mathrm{VDCR}^{+} \mathrm{CGCT}^{+}$and $\mathrm{Pwi}^{+/+} \mathrm{VDCR}^{+} \mathrm{CGCT}^{+}$mice have 
comparable RRP sizes, but almost a two-fold difference in the sustained rates.
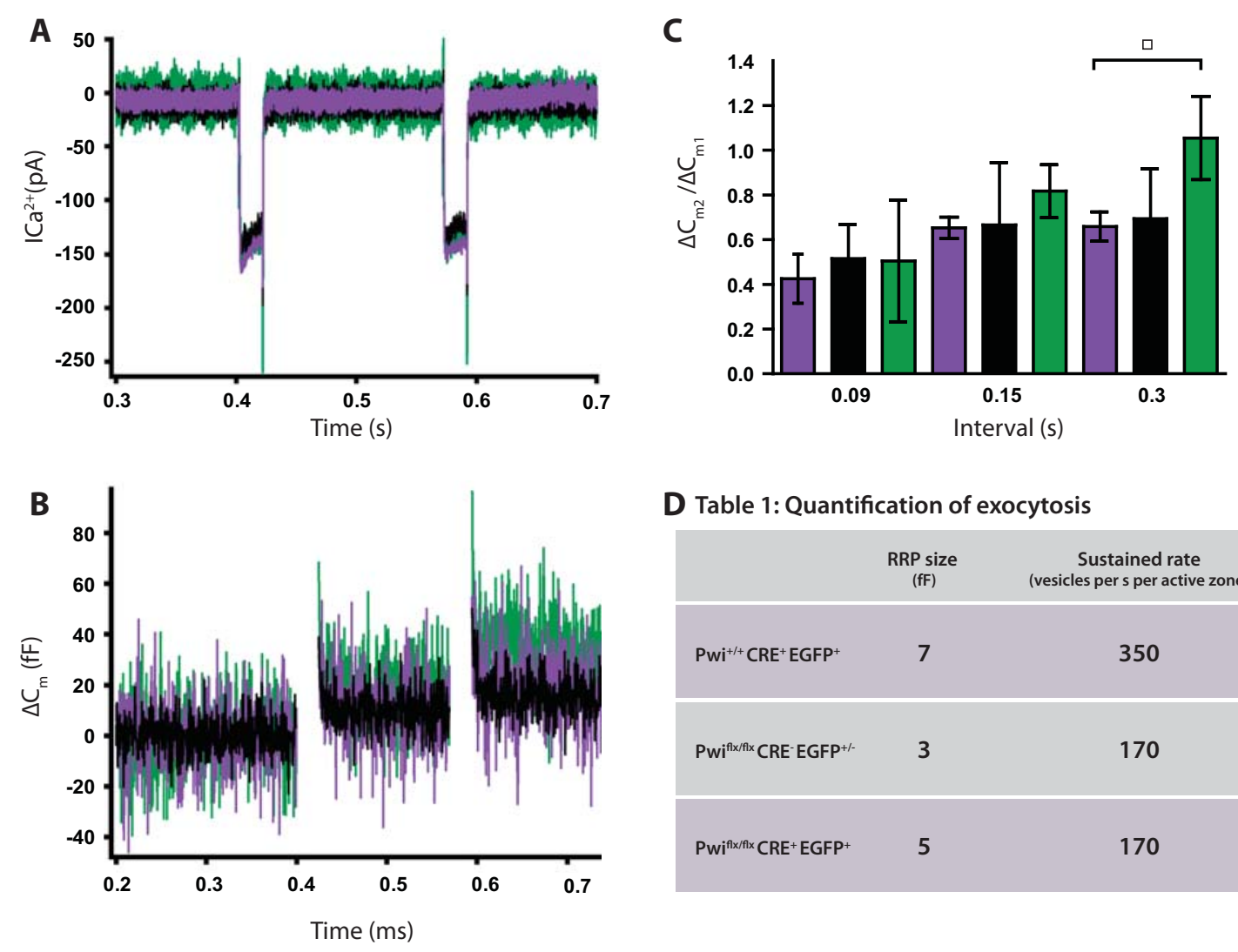

D Table 1: Quantification of exocytosis

\begin{tabular}{|c|c|c|}
\hline & $\begin{array}{l}\text { RRP size } \\
\text { (fF) }\end{array}$ & $\begin{array}{l}\text { Sustained rate } \\
\text { (vesicles per s per active zone) }\end{array}$ \\
\hline $\mathrm{Pwi}^{+/+} \mathrm{CRE}^{+} \mathrm{EGFP}^{+}$ & 7 & 350 \\
\hline Pwif ${ }^{\text {fl/fflx }}$ CRE- EGFP $^{+1-}$ & 3 & 170 \\
\hline $\mathrm{Pwi}^{\mathrm{ffx} / \mathrm{flx}} \mathrm{CRE}^{+} \mathrm{EGFP}^{+}$ & 5 & 170 \\
\hline
\end{tabular}

Figure 3.8: Synaptic vesicle replenishment of $\mathrm{Pwi}^{\mathrm{flx} / \mathrm{flx}} \mathrm{VDCR}^{+} \mathrm{CGCT}^{+} \mathrm{IHCs}$ is slowed down. (A) Representative $\mathrm{Ca}^{2+}$ currents and (B) $\mathrm{C}_{m}$ changes in response to a pair of $20 \mathrm{~ms}$ depolarizations, separated by $150 \mathrm{~ms}$. (C) Mean ratios ( \pm s.e.m) of $\mathrm{C}_{m}$ response of the second depolarization $\left(\Delta \mathrm{C}_{m}\right)$ over the first $\left(\Delta \mathrm{C}_{m} 1\right)$ as a function of interstimulus intervals, $0.09 \mathrm{~s}, 0.15$ $\mathrm{s}$ and $0.3 \mathrm{~s}$. At $0.3 \mathrm{~s}$ interstimulus time interval the recovery of exocytosis in $\mathrm{Pwi}^{\mathrm{fx} / \mathrm{fl} \mathrm{x}} \mathrm{VDCR}^{+}$ $\mathrm{CGCT}^{+}$IHCs was significantly reduced as compared to $\mathrm{Pwi}^{+/+} \mathrm{VDCR}^{+} \mathrm{CGCT}^{+}$IHCs. $\square \mathrm{p}=$ 0.03 (t-test, see details in the text). (D) Table 1: Quantification of exocytosis

The observed reduction in the sustained component of the release in $\mathrm{Pwi} \mathrm{flx}^{\mathrm{fl}} \mathrm{fl}$ $\mathrm{VDCR}^{+} \mathrm{CGCT}^{+}$IHCs prompted me to further investigate the RRP recovery from depletion using a paired-pulse stimulation. Here, the first depolarizing stimulus triggers exocytosis and depletes the pool of RRP vesicles. Waiting for various time intervals $(90,150,300 \mathrm{~ms})$ is expected to allow partial or complete replenishment of the pool, which gets depleted again during a second depolarizing pulse. Depending on the initial depletion of the pool of vesicles and the ability of the cell to 
replenish this pool during the interpulse interval, the cell's replenishment rate can be evaluated by the second stimulus. RRP recovery was assessed as the ratio of the membrane capacitance difference after the second pulse $\left(\Delta \mathrm{C}_{m} 2\right)$ to the capacitance difference after the first pulse $\left(\Delta \mathrm{C}_{m} 1\right)$ for the various inter-stimulus intervals. Examples of calcium currents and corresponding membrane capacitance changes during a paired-pulse stimulation with a $150 \mathrm{~ms}$ interstimulus interval are represented in Figure 3.8 A and B. The replenishment rates are shown in Figure 3.8 C. Significant difference was observed between $\mathrm{Pwi}^{\mathrm{flx} / \mathrm{flx}} \mathrm{VDCR}^{+} \mathrm{CGCT}^{+}$and $\mathrm{Pwi}^{+/+} \mathrm{VDCR}^{+}$ $\mathrm{CGCT}^{+}$at $0.3 \mathrm{~s}(\mathrm{p}=0.03)$, which is consistent with the observation of the reduced sustained component of release. Reductions in the paired-pulse ratios were observed at other interstimulus intervals and for the $\mathrm{Pwi}^{\mathrm{flx} / \mathrm{flx}} \mathrm{VDCR}^{-} \mathrm{CGCT}^{+}$genotype as well, however the differences did not reach statistical significance. This might be due to relatively high variability in these demanding experiments, where a proper depletion of the RRP pool during the first pulse was not always achieved.

\subsection{Synaptic morphology}

\subsubsection{Indication of a progressive reduction in the number of ribbon- occupied synapses of $\mathrm{Pwi}^{\mathrm{fx} / \mathrm{flx}} \mathrm{VDCR}^{+} \mathrm{CGCT}^{+} \mathrm{IHCs}$}

To test if the perturbation of the WRB protein in any way affects the number of ribbon synapses, I further performed histological examination and analysis of the apical coils of organs of Corti for all the three genotypes in age groups I and II by means of immunolabeling. Antibodies against Ribeye/CtBP2, a major structural component of the ribbon, and against GluA subunits $2 / 3$, the post-synaptic AMPA receptors, were used to stain the afferent ribbon synapse. Intact ribbon synapses were identified by the presence of closely juxtaposed Ribeye/CtBP2 and GluA2/3 fluorescent spots (Figure 3.9, CtBP2 in red and GluA2/3 in green). I quantified the number of IHC ribbon synapses by counting the juxtaposed pre- and postsynaptic fluorescent spots of CtBP2 and GluA2/3 in maximum projections (stacks) of confocal sections, divided by the number of nuclei in each stack. The results (Figure 3.9 Table 2) show that the number of ribbon synapses in all three genotypes 
at age group I are similar, whereas there is an indication of age-dependent reduction in synapses of $\mathrm{Pwi}^{\mathrm{flx} / \mathrm{fx}} \mathrm{VDCR}^{+} \mathrm{CGCT}^{+}$IHCs.

\subsubsection{Unusually large vesicles accumulate at the $\mathrm{KO}$ synapse}

To gain more insight into the morphology of the IHC ribbon synapses, I, in collaboration with Dr. Carolin Wichmann, investigated the ribbon synapse ultrastructure by means of electron microscopy. For the preliminary data set, embeddings of 1 organ of Corti from $\mathrm{Pwi}^{\mathrm{flx} / \mathrm{flx}} \mathrm{VDCR}^{+} \mathrm{CGCT}^{+}$animal and 1 from $\mathrm{Pwi}^{\text {flx }} / \mathrm{flx} \mathrm{VDCR}^{-}$ $\mathrm{CGCT}^{+}$animal were performed at room temperature and sections of $70 \mathrm{~nm}$ were taken from multiple IHCs. In all IHCs of the $\mathrm{Pwi}^{\mathrm{fx} / \mathrm{flx}} \mathrm{VDCR}^{+} \mathrm{CGCT}^{+}$animal and in some IHCs of the $\mathrm{Pwi}^{\mathrm{flx} / \mathrm{flx}} \mathrm{VDCR}^{-} \mathrm{CGCT}^{+}$control animal, unusually large vesicle-like structures, with diameter $>55 \mathrm{~nm}$, were observed around the ribbon or in close proximity to the synapse (Figure 3.10 a-f and Figure $3.11 \mathrm{~g}$ ). Such vesicles often formed clusters (Figure $3.10 \mathrm{c}, \mathrm{d}, \mathrm{f}$ ). Most ribbons in $\mathrm{Pwi}^{\mathrm{flx} / \mathrm{flx}} \mathrm{VDCR}^{+}$ $\mathrm{CGCT}^{+}$IHCs were intact whereas some of them appeared to be partially disrupted, showing a non-uniform shape (Figure $3.10 \mathrm{c}$ and Figure $3.11 \mathrm{~g}$ ). However, even such ribbons tethered synaptic vesicles, of which some were found close to the plasma membrane and were likely docked. All ribbons were also attached to the plasma membrane. Furthermore, tubular structures with ribosomes on them (Figure 3.10 $\mathrm{d}$ and also in e and $\mathrm{f}$ without ribosomes) were often observed parallel to the presynaptic plasma membrane at $\mathrm{Pwi}^{\mathrm{flx} / \mathrm{flx}} \mathrm{VDCR}^{+} \mathrm{CGCT}^{+}$. Although the nature and origin of these vesicles is puzzling, it is tempting to speculate that they might be related to cellular efforts to compensate for a deficit of TA protein insertion into the membrane.

Vesicles around the ribbons within $500 \mathrm{~nm}$ radius from the active zone were quantified for their size. Inside this area, the diameter of the vesicles was measured and they were then categorized into three groups: ribbon-associated vesicles, vesicles between the ribbon and the presynaptic membrane (presumably docked), and vesicles in the rest area of the $500 \mathrm{~nm}$. Ribbon-associated vesicles I considered those vesicles that were found in immediate apposition of the ribbon, had a circular shape, and had an average diameter of 35-45 nm. I found a significant difference $(\mathrm{p}=0.01)$ 


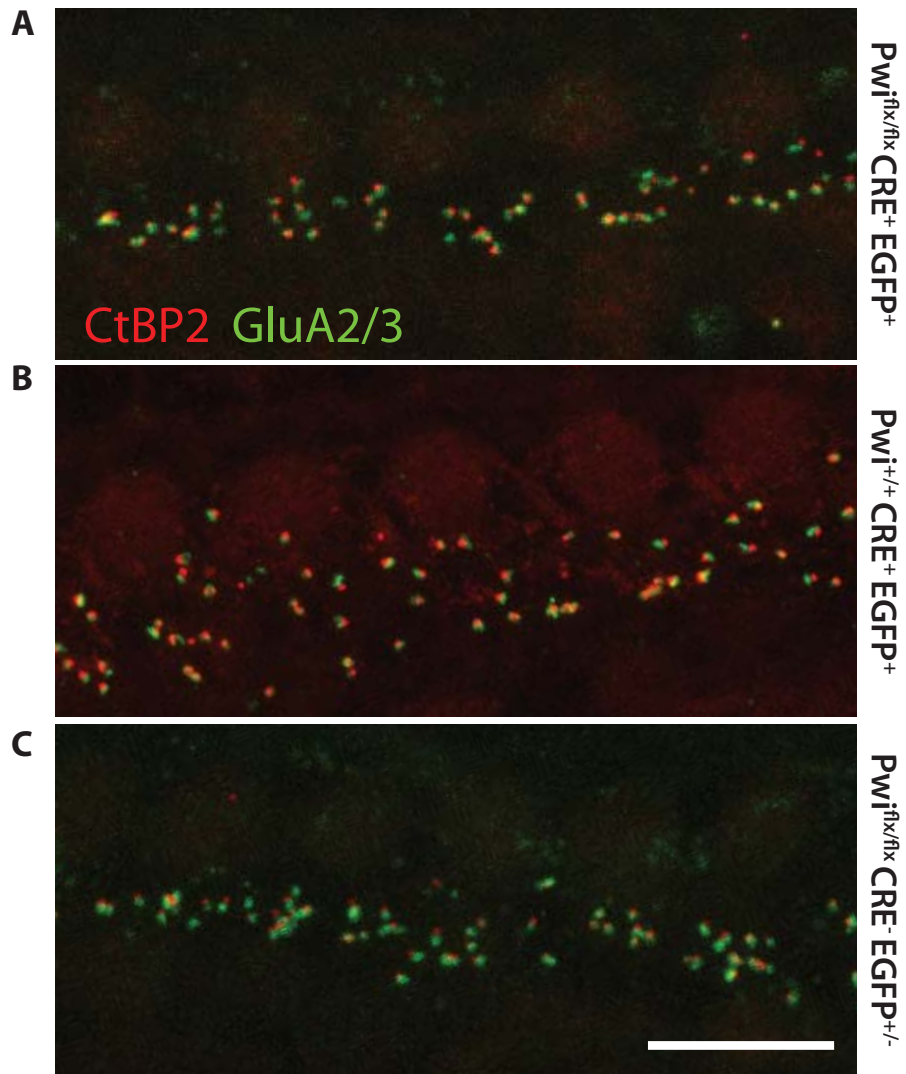

\begin{tabular}{|c|c|c|c|}
\hline & Pwif $^{\mathrm{nx} / \mathrm{fl} \mathrm{C}} \mathrm{CRE}^{+} \mathrm{EGFP}^{+}$ & $\mathrm{Pwi}^{+++} \mathrm{CRE}^{+}$EGFP $^{+}$ & Pwif $^{\mathrm{nx} / \mathrm{fl} \mathrm{C}}$ CRE EGFP $^{+/-}$ \\
\hline & \multicolumn{3}{|c|}{ Age Group I (P14 - P19) } \\
\hline No. of investigated IHCs & 140 & 119 & 93 \\
\hline No. of synapses/cell & $12.12 \pm 1.65$ & $9.6 \pm 0.88$ & $11.13 \pm 1.92$ \\
\hline No. of CtBP2 spots/cell & $12.64 \pm 1.72$ & $10.2 \pm 0.96$ & $12.3 \pm 1.76$ \\
\hline \multirow[t]{2}{*}{ No. of GluA2/3 spots/cell } & $12.77 \pm 1.81$ & $10.17 \pm 1.03$ & $15 \pm 4.15$ \\
\hline & \multicolumn{3}{|c|}{ Age Group II (P21 - P29) } \\
\hline No. of investigated IHCs & 16 & 53 & 106 \\
\hline No. of synapses/cell & 7 & $10.2 \pm 1.8$ & $12.2 \pm 2.0$ \\
\hline No. of CtBP2 spots/cell & 8.06 & $9.8 \pm 0.3$ & $13 \pm 1.9$ \\
\hline No. of GluA2/3 spots/cell & 16.8 & $10.04 \pm 2.2$ & $12.7 \pm 2$ \\
\hline
\end{tabular}

Figure 3.9: Quantification of ribbon synapses. Representative example projections of confocal sections of IHCs (age group I) following immunolabeling of ribbons (anti-CtBP2, red) and glutamate receptors (anti-GluA2/3, green). (A) $\mathrm{Pwi}^{\mathrm{fx} / \mathrm{flx}} \mathrm{VDCR}^{+} \mathrm{CGCT}^{+}$, (B) $\mathrm{Pwi}^{+/+} \mathrm{VDCR}^{+}$ $\mathrm{CGCT}^{+}$, and $(\mathrm{C}) \mathrm{Pwi}^{\mathrm{flx} / \mathrm{flx}} \mathrm{VDCR}^{-} \mathrm{CGCT}^{+}$. Scale bar is $10 \mu \mathrm{m}$. Table 2: Quantification of ribbon synapses 
in the number of "large vesicles" (with diameter $>55 \mathrm{~nm}$ ) at the synapses of the $\mathrm{Pwi}^{\mathrm{flx} / \mathrm{flx}} \mathrm{VDCR}^{+} \mathrm{CGCT}^{+}(4.1 \pm 0.8)$ compared to the Pwifle/flx $\mathrm{VDCR}^{-} \mathrm{CGCT}^{+}$ control $(1.4 \pm 0.3)$ (Figure $3.12 \mathrm{~A}$ ) and almost a two-fold reduction $(\mathrm{p}=0.001)$ in
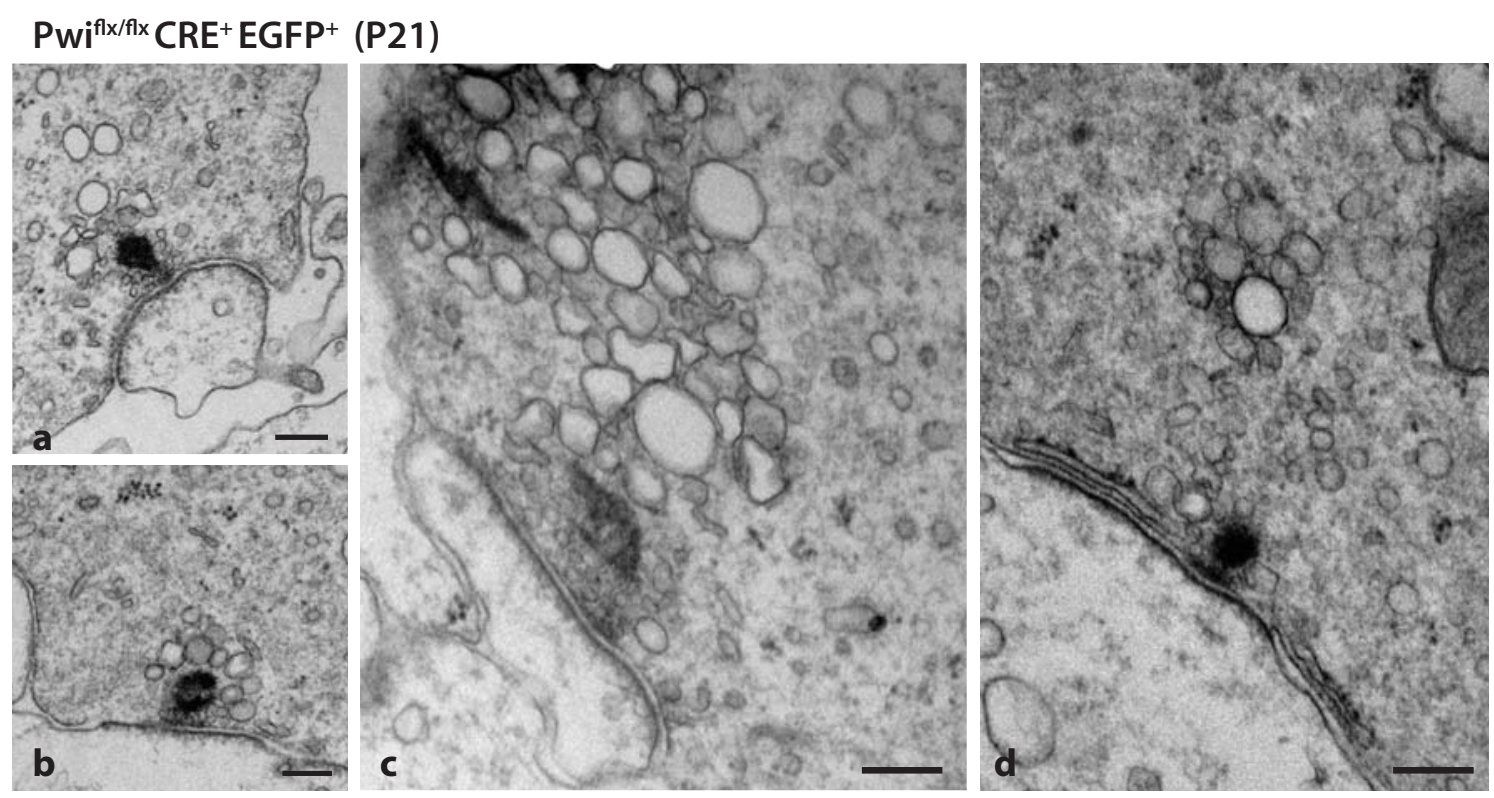

\section{$\mathrm{Pwi}^{\mathrm{ffl} / \mathrm{fl} \times} \mathrm{CRE}^{+} \mathrm{EGFP}^{+}(\mathrm{P} 14)$}
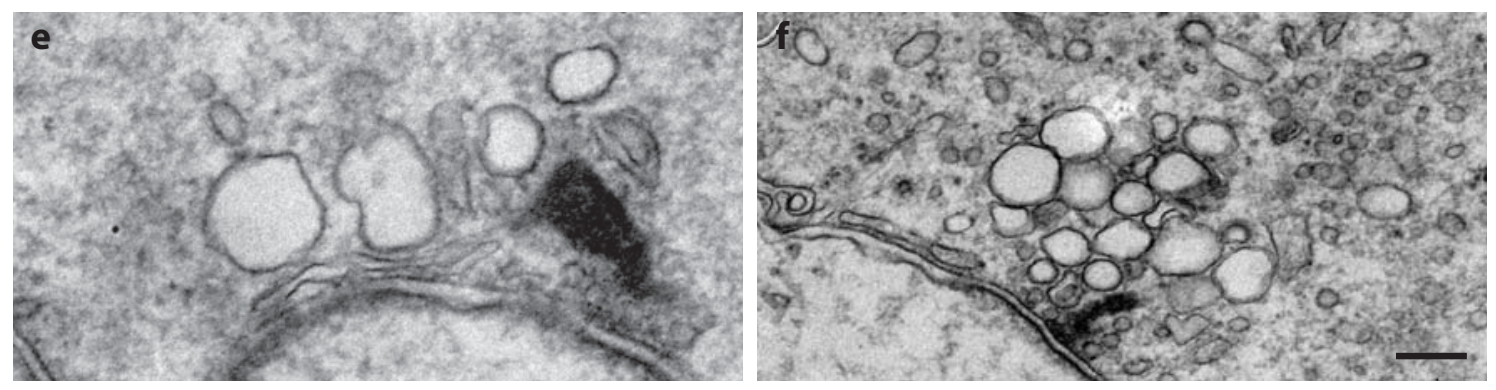

Figure 3.10: Ultrastructural analysis of the $\boldsymbol{P} \boldsymbol{w i}$ ribbon synapse. Transmission electron micrographs of IHC ribbon synapses in $\mathrm{Pwi}^{\mathrm{fx} / \mathrm{flx}} \mathrm{VDCR}^{+} \mathrm{CGCT}^{+}$animal P21 (a-d) and P14 (e-f). Scale bars are $200 \mathrm{~nm}$.

the number of ribbon-associated vesicles (diameter $\sim 45 \mathrm{~nm}$ ) in $\mathrm{Pwi}^{\mathrm{flx} / \mathrm{flx}} \mathrm{VDCR}^{+}$ $\mathrm{CGCT}^{+} \operatorname{IHCs}\left(5.5 \pm 0.2\right.$ and $\mathrm{Pwi}^{\mathrm{flx} / \mathrm{flx}} \mathrm{VDCR}^{-} \mathrm{CGCT}^{+}: 9.7 \pm 0.6$ ) (Figure 3.12 B). The number of the ribbon-associated vesicles in the $\mathrm{Pwi}^{\mathrm{fx} / \mathrm{flx}} \mathrm{VDCR}^{-} \mathrm{CGCT}^{+}$ was similar to the average number found at the IHC ribbon synapses of the WT C57Bl/6J mice (Dr. Carolin Wichmann personal communication).

In summary, synapse number was normal in Pwi KO IHCs at the age group 
$\mathrm{Pwi}^{\mathrm{fl} / \mathrm{fl} \mathrm{x}}$ CRE$^{-} \mathrm{EGFP}^{+/-}(\mathrm{P} 21)$
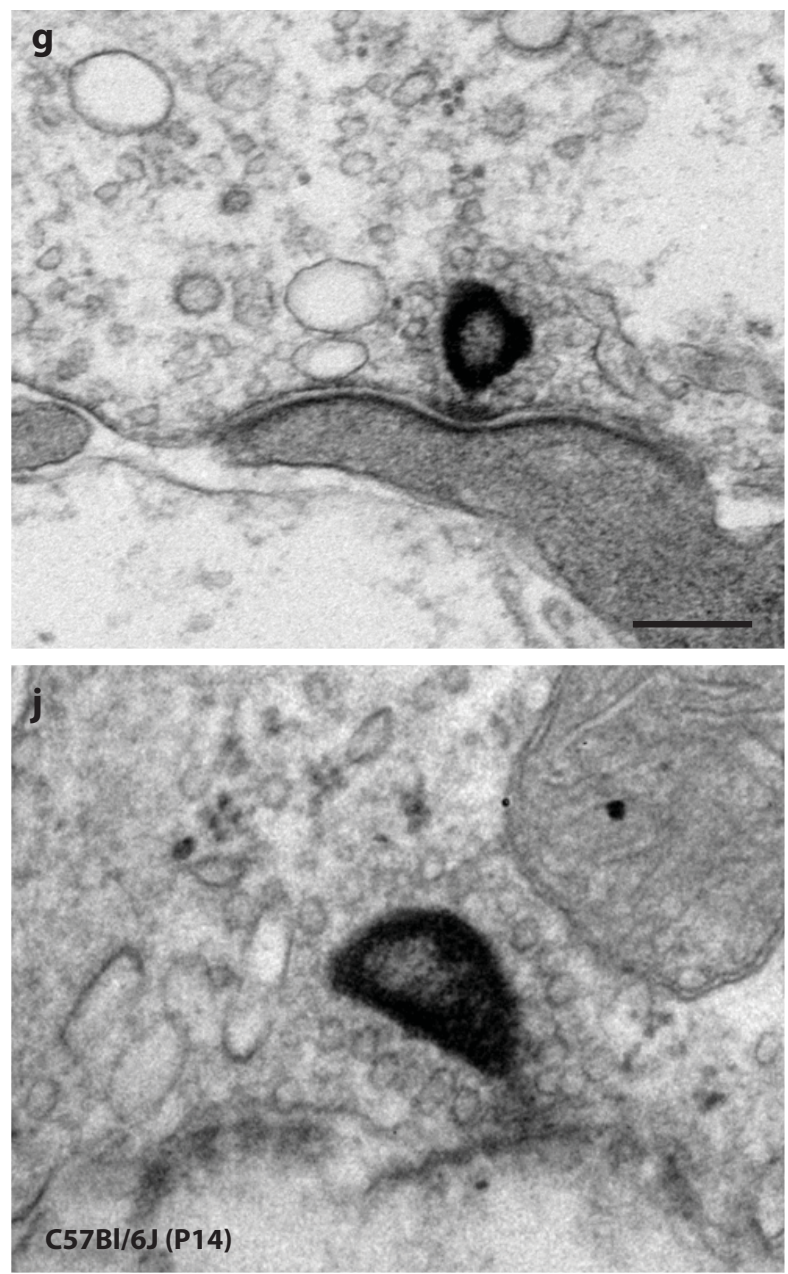
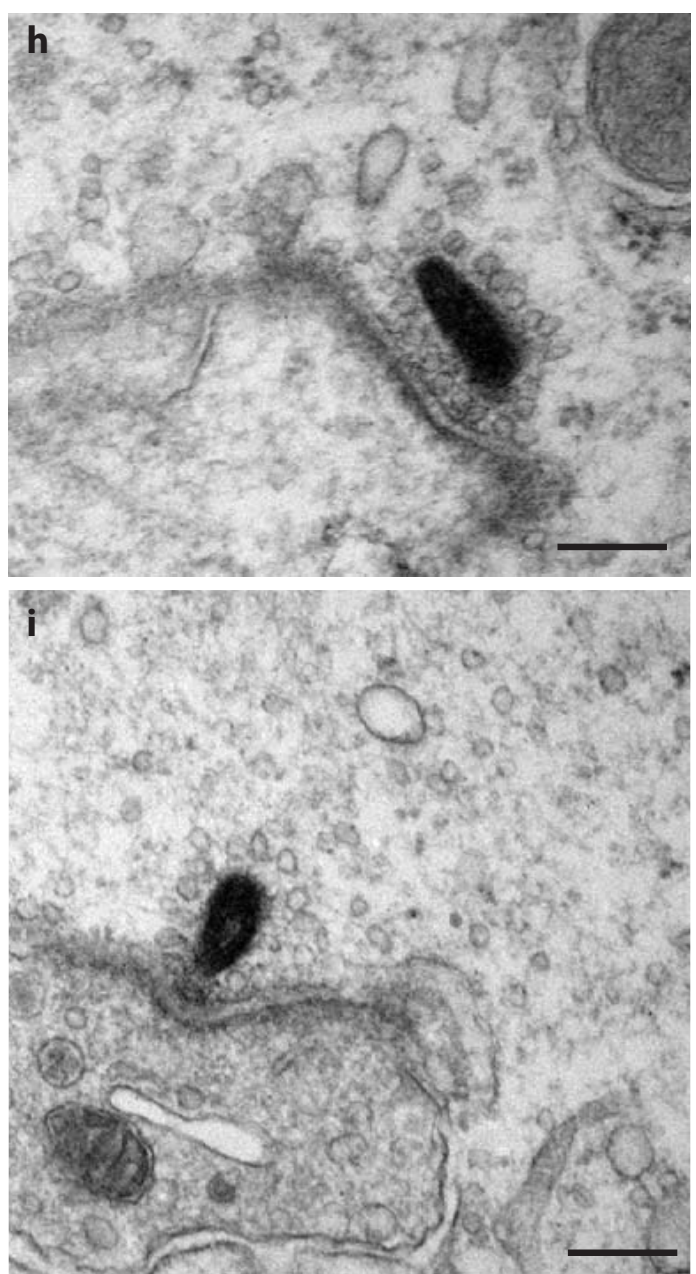

Figure 3.11: Ultrastructural analysis of the Pwi ribbon synapse (controls). Transmission electron micrographs of IHC ribbon synapses in $\mathrm{Pwi}^{\mathrm{fx} / \mathrm{flx}} \mathrm{VDCR}^{-} \mathrm{CGCT}^{+}$animal P21 (g-i) and one example C57Bl/6J P14 (j). Scale bars are $200 \mathrm{~nm}$.

I. Analysis of the ribbon synapse morphology revealed unusual vesicle and ribbon patterns. IHCs of $\mathrm{Pwi}^{\mathrm{flx} / \mathrm{flx}} \mathrm{VDCR}^{-} \mathrm{CGCT}^{+}$mice showed impaired exocytosis and some morphological abnormalities of IHC ribbon synapses. 
A

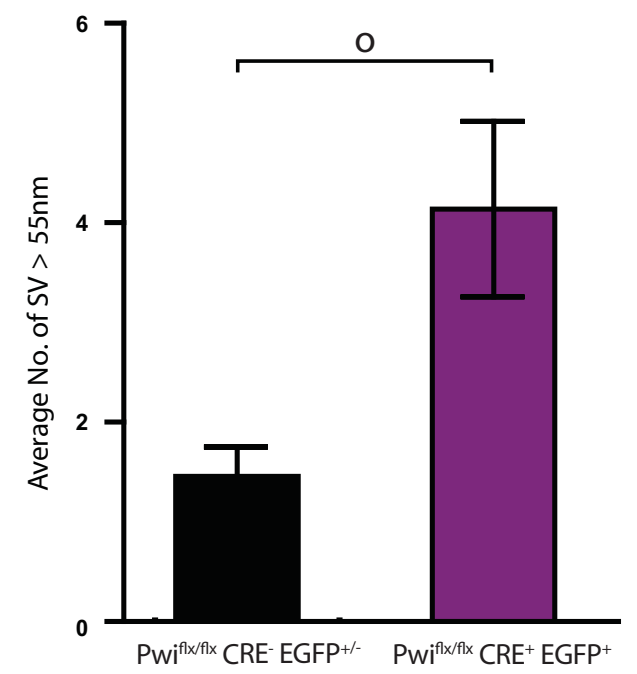

B

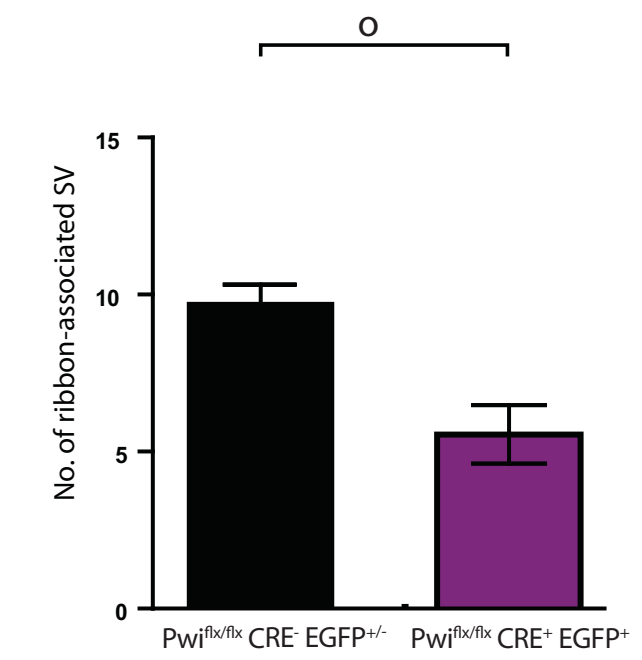

Figure 3.12: Quantification of ultrastructural analysis of the IHC ribbon synapses. (A) Average number of SV with diameter $>55 \mathrm{~nm}$ in the two genotypes studied (B) Average number of ribbon-associated SV in the two genotypes. Two-tailed paired T-test was applied. o denotes the significant difference between $\mathrm{Pwi}^{\mathrm{flx} / \mathrm{flx}} \mathrm{VDCR}^{+} \mathrm{CGCT}^{+}$(violet) and $\mathrm{Pwi}^{\mathrm{fx} / \mathrm{flx}} \mathrm{VDCR}^{-} \mathrm{CGCT}^{+}$ (black). Data are represented as mean \pm s.e.m.

\subsection{Effect of the $W r b$ deletion on the molecular composition of the IHC ribbon synapse}

Where does WRB localize to in the IHC? Does it act as a receptor of tail-anchored (TA) proteins and/or does it participate more actively at the synaptic site? How does Wrb deletion affect the expression levels and trafficking of the TA protein otoferlin in IHCs? These questions are the key for deciphering the role of WRB in the IHCs.

\subsubsection{Reduced otoferlin protein levels}

I first addressed the question whether the protein levels of otoferlin are affected in the IHCs of the Pwi KO. Both, expression levels and localization pattern of otoferlin were studied by semi-quantitative confocal microscopy of immunolabeled IHCs. Apical coils of organs of Corti of all the 3 genotypes and age groups were 
immunolabeled with antibodies against the N-terminus of otoferlin and VGLUT3 and confocal sections were acquired using the same settings (Figure 3.14).
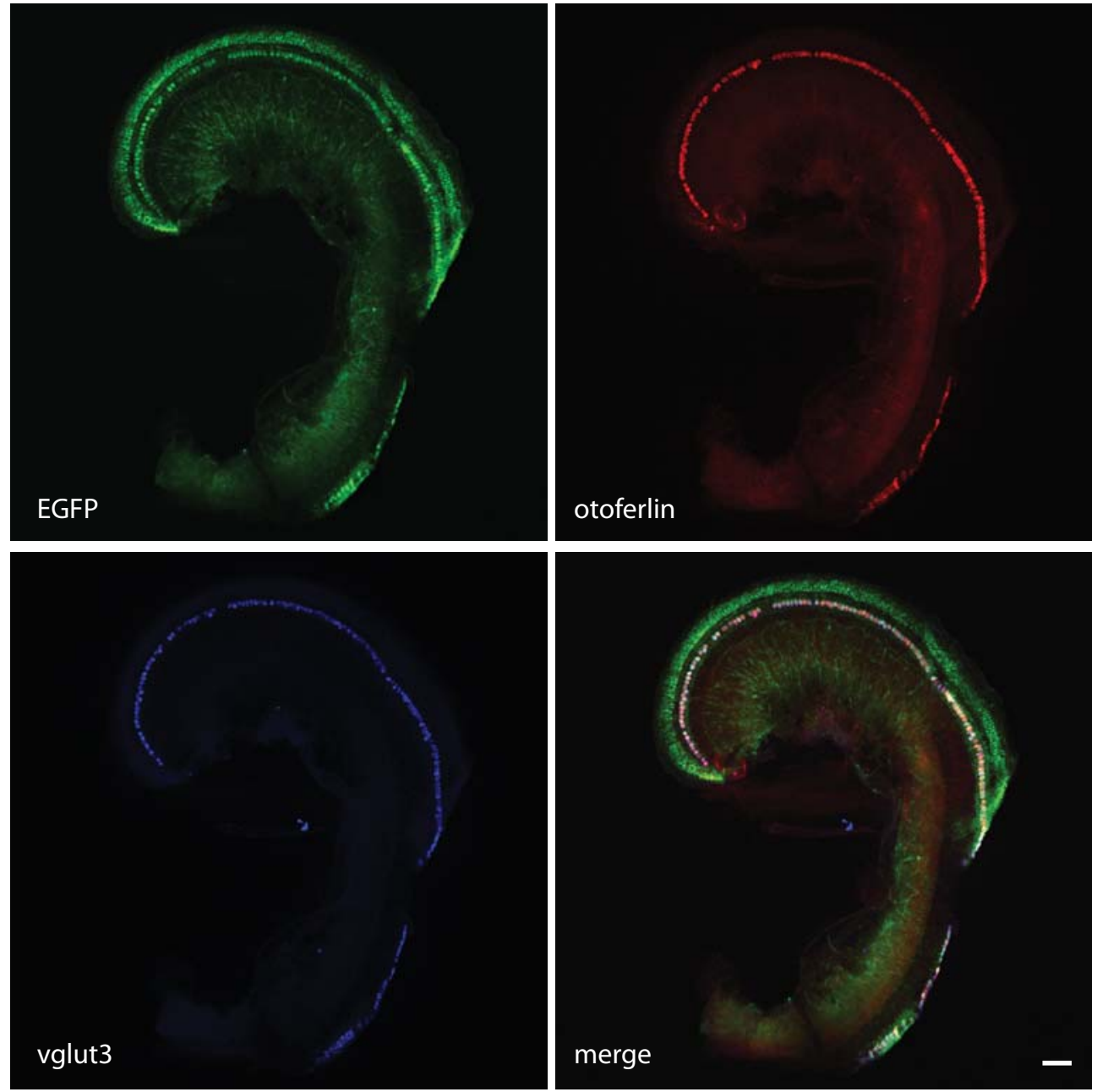

Figure 3.13: Expression pattern of otoferlin distribution in the first cochlear turn. Characteristic examples of images from single confocal sections of an apical cochlear turn of a $\mathrm{Pwi}^{\mathrm{fx} / \mathrm{flx}} \mathrm{VDCR}^{+} \mathrm{CGCT}^{+}$animal. Expression patterns of: endogenous EGFP, as a result of the Cre activity, otoferlin and VGLUT3 after immunolabeling with specific antibodies.

A custom macro, written by Dr. Tina Pangršič was used to quantify the fluorescence signal and investigate the subcellular distribution of otoferlin and VGLUT3. The results show a tendency toward lower otoferlin fluorescence in the IHCs of the $\mathrm{Pwi}^{\mathrm{flx} / \mathrm{flx}} \mathrm{VDCR}^{+} \mathrm{CGCT}^{+}$mice at all ages, both in apical (Figure $3.15 \mathrm{~A}$ ) as well as in basal parts of the cells (Figure $3.15 \mathrm{~B}$ ); however, more data are required to definitely confirm or reject this observation. Interestingly, the fluorescence in the 
$\mathrm{Pwi}^{\mathrm{ifl} / \mathrm{flx}} \mathrm{CRE}^{+} \mathrm{EGFP}^{+}$
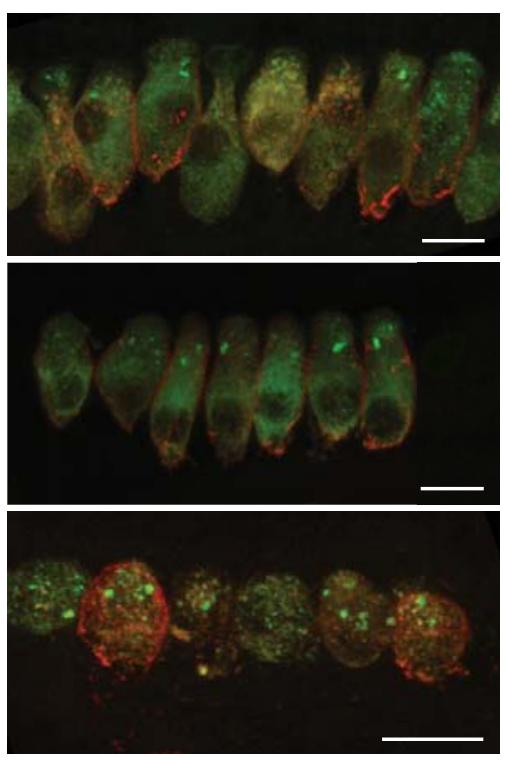

$\mathrm{Pwi}^{+/+} \mathrm{CRE}^{+} \mathrm{EGFP}^{+}$
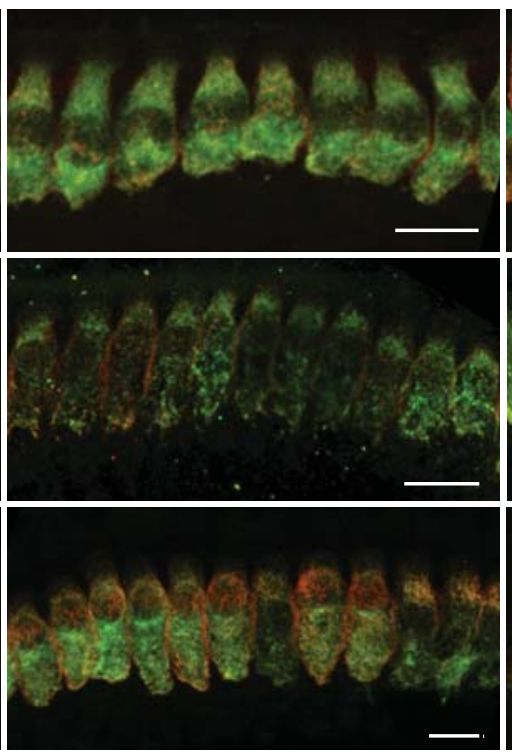

Pwi $^{\mathrm{ifl} / \mathrm{fl} x}$ CRE- EGFP ${ }^{+-}$

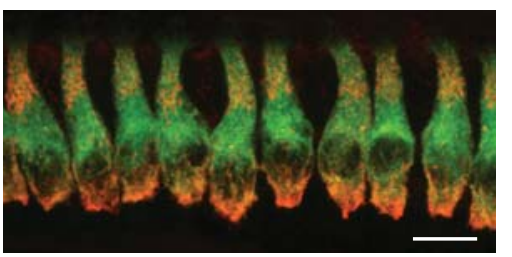

I
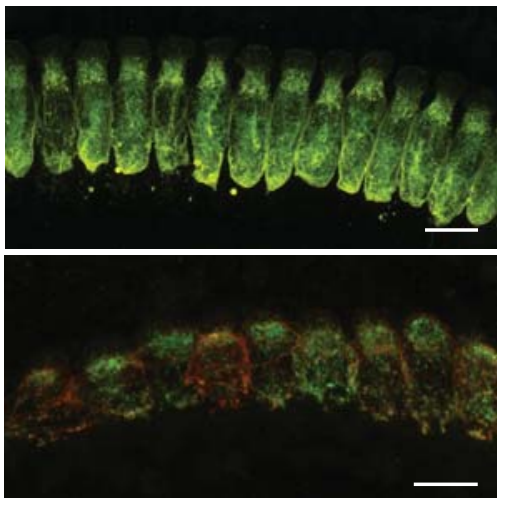

Figure 3.14: Expression pattern of otoferlin distribution in apical cochlear IHCs. Maximum projections of confocal Z sections of apical IHCs of the three different genotypes and age groups, following immunolabeling for otoferlin (in red) and for VGLUT3 (in green), processed and imaged under the same conditions. Scale bar is $10 \mu \mathrm{m}$.

control $\mathrm{Pwi}^{\mathrm{flx} / \mathrm{flx}} \mathrm{VDCR}^{-} \mathrm{CGCT}^{+}$IHCs seems to be stronger as compared to the control Pwi ${ }^{+/+} \mathrm{VDCR}^{+} \mathrm{CGCT}^{+}$IHCs.

\subsubsection{Disruption of the protein WRB changes the distribution of the TRC40 interaction partner}

In mammalian cells, Wrb has been proposed to act as a receptor of TA proteins (Vilardi et al., 2011). The pathway mediating insertion of TA proteins to the ER membrane demands a cytosolic factor, TRC40, that can deliver the newly synthesized polypeptide to the ER receptor. The interaction of TRC40 with WRB is an important step for the insertion of the protein to the ER (Yamamoto and Sakisaka, 2012). Upon deletion of $W r b$, the TRC40 protein membrane insertion pathway is likely disturbed. I tested for TRC40 expression in IHCs by immunohistochemistry in Wrb KO IHCs, using a TRC40 antibody, generated and kindly provided by Dr. Fabio Vilardi (Favaloro et al., 2010). The pattern of distribution and the expres- 
A
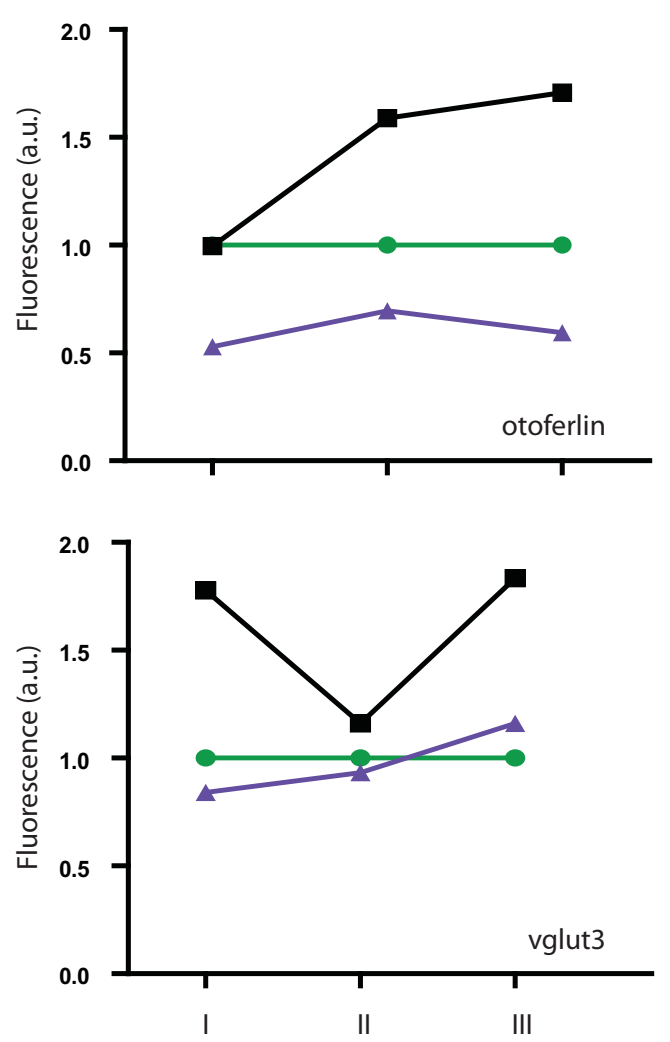

Age Group
B
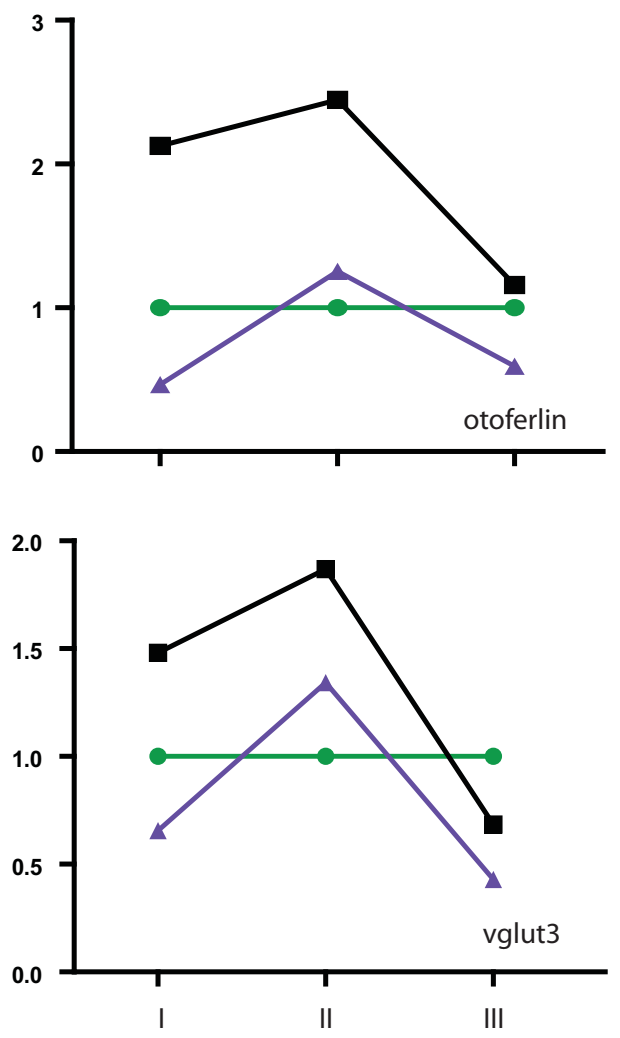

Age Group

$\mathrm{Pwi}^{+/+} \mathrm{CRE}^{+} \mathrm{EGFP}^{+}$

Pwi ${ }^{f 1 / f l x} C^{-} E^{-} E_{F F}^{+/-}$

$\simeq \mathrm{Pwi}^{\mathrm{f} \mathrm{f} / \mathrm{fl} \mathrm{x}} \mathrm{CRE}^{+} \mathrm{EGFP}^{+}$

Figure 3.15: Semi-quantitative evaluation of otoferlin protein levels. Average fluorescence intensity of otoferlin (upper graph) and VGLUT3 (lower graph) for the (A) apical part and (B) basal part of IHCs of all three genotypes and age groups. In the Y axis, the fluorescence values are presented in arbitrary units (a.u.).

sion levels were examined in apical IHCs of two $\mathrm{Pwi}^{\mathrm{fx} / \mathrm{flx}} \mathrm{VDCR}^{-} \mathrm{CGCT}^{+}$, and two $\mathrm{Pwi}^{\mathrm{fl} / \mathrm{flx}} \mathrm{VDCR}^{+} \mathrm{CGCT}^{+}$animals. Otoferlin antibody was used as an IHC marker.

The data indicate that TRC40 is indeed expressed in mouse IHCs and mostly localizes to the cytoplasm of the basal part. On the contrary, TRC40 was almost absent from the basal part of IHCs in the $\mathrm{Pwi}^{\mathrm{flx} / \mathrm{flx}} \mathrm{VDCR}^{+} \mathrm{CGCT}^{+} \mathrm{IHCs}$, in agreement with the initial hypothesis, while a faint signal remained in the very apical part of the cells, close to the hair bundles (Figure 3.16). 

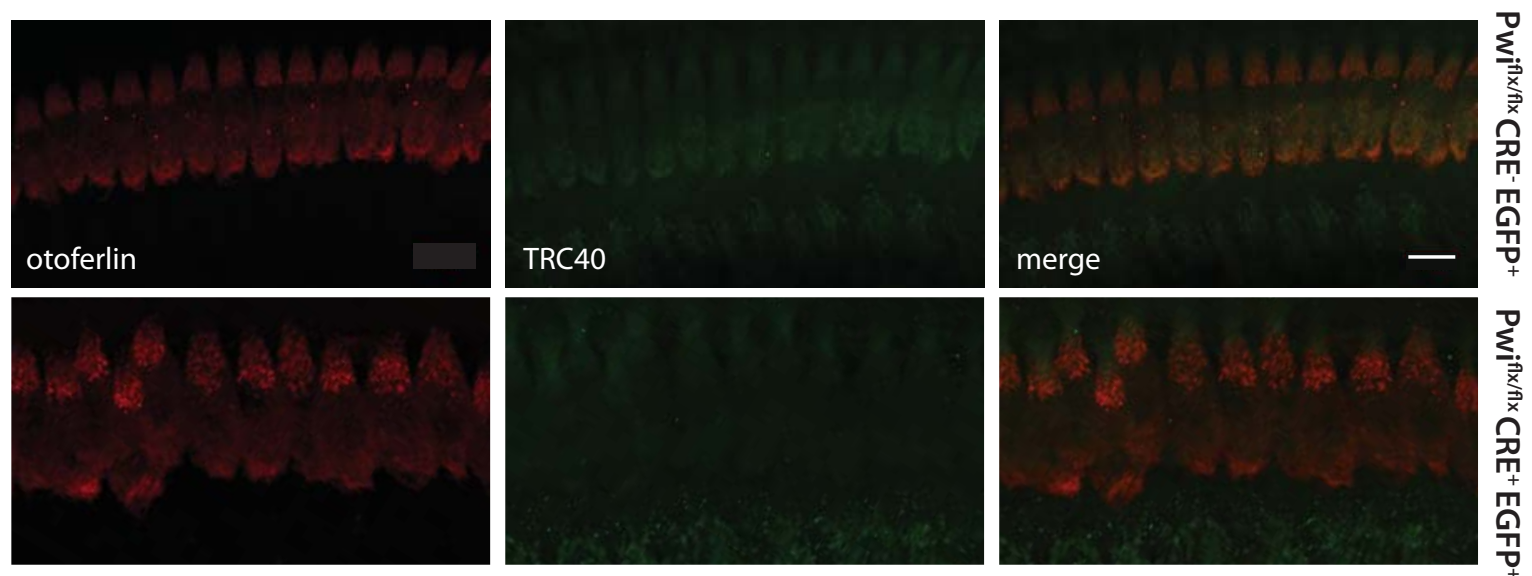

Figure 3.16: Reduced expression of TRC40. Maximum projections of otoferlin and TRC40 staining in apical IHCs. Scale bar: $10 \mu \mathrm{m}$.

\subsection{Silencing of otoferlin}

Previous data in otoferlin mutant mouse model suggested that the perturbation of otoferlin might affect the synaptic function of IHCs in a dose-dependent manner (Pangršič et al., 2010). Our results in the pinball-wizard KO IHCs, demonstrating a reduction in sustained component of IHC exocytosis together with a reduction in otoferlin levels, also support this notion. Therefore, I aimed at testing this hypothesis by selectively reducing the expression levels of otoferlin, without concomitant deletion of another protein (like WRB) or putative changes in the structure of the otoferlin protein (like discussed for the pachanga mutants). To this aim, I proposed to use two approaches: first, a silencing of the expression of otoferlin, and second, to investigate synaptic function in otoferlin heterozygous animals, which also show partially reduced levels of otoferlin in IHCs (Pangršič et al., 2010).

A knock-down system that would allow the disruption of expression of the various proteins in a relatively easy and quick way, without the generation of transgenic mouse lines, could become an exceptionally useful tool. My purpose was therefore to establish an IHC-targeted silencing system, which I planned to first use to manipulate the expression levels of otoferlin. 
A

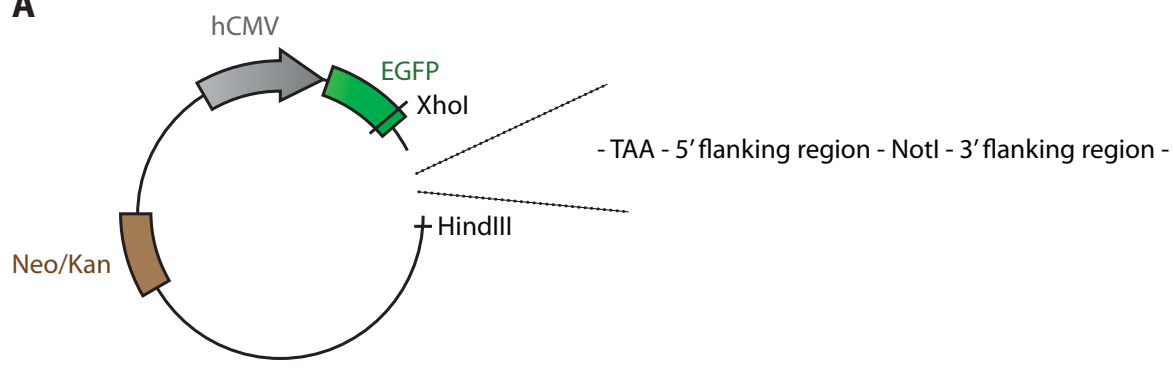

B
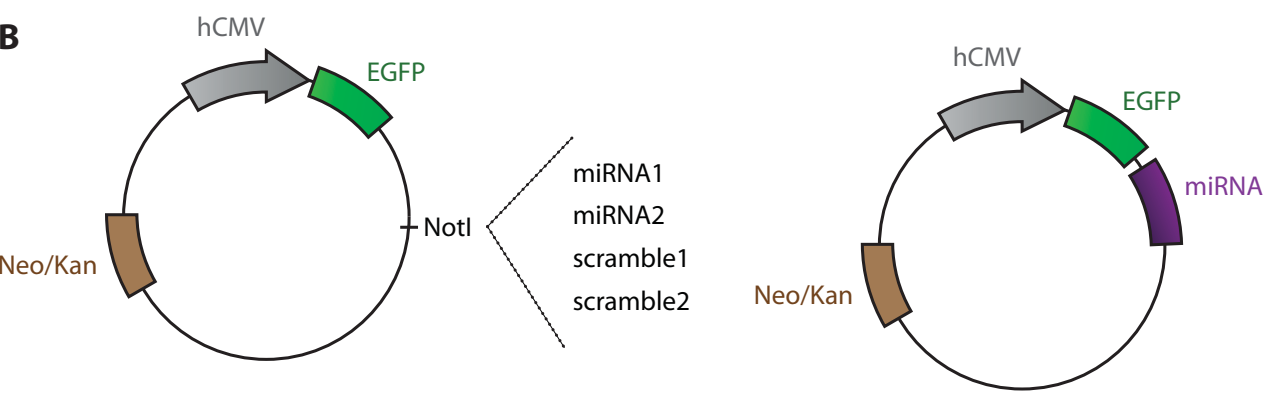

Figure 3.17: Construction of the silencing vectors. (A) Illustration of the map of the original backbone vector, pEGFP-C1, which was used for insertion of the miRNA-like silencing oligomers. The endonuclease restriction sites XhoI and HindIII indicate the position of the insertion of the microtemplate cassette, as described in the chapter Materials and Methods. (B) The mammalian vector in which the individual miRNA-like silencing molecules were cloned downstream of EGFP.

\subsubsection{In vitro silencing}

Initially, I screened 8 different (4 silencing and 4 scramble) miRNA vectors for their transfection efficiency and for any possible toxicity in HEK 293T mammalian cells. Very high transfection levels ( $90 \%)$ were indicated by the EGFP fluorescent positive cells and no cell toxicity was observed in DAPI staining (not shown). Next, in order to assess the silencing capability of the individual miRNAs, I performed double-plasmid transfections in HEK cells and quantified the protein levels by western blot analysis. Full-length mouse otoferlin cDNA, expressed by a pEGFP-N1 vector (produced by Dr. Ellen Reisinger), was transfected together with one or two miRNA vectors into HEK cells. Multiple combinations of plasmid concentrations were tested, using Lipofectamine 2000 and calcium phosphate transfection protocols. In the quantification presented in Figure 3.18, $3 \mu \mathrm{g}$ of DNA were used in single 

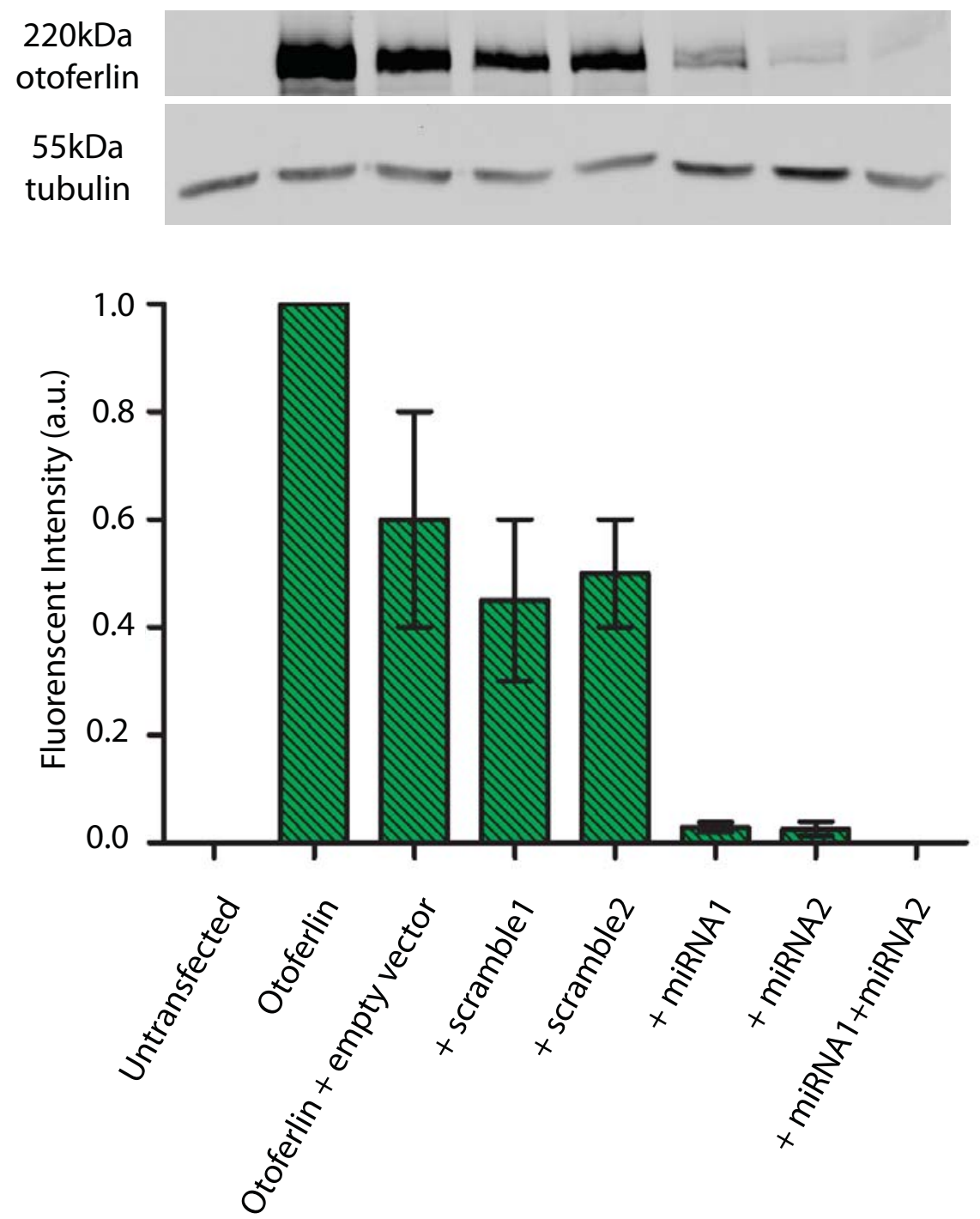

Figure 3.18: In vitro silencing - Protein level quantification. Western blot analysis for otoferlin in HEK 293T cells: untransfected cells, transfected with otoferlin vector, double transfection with otoferlin vector and a gene-empty vector, otoferlin vector and pEGFP-C1scramble1, otoferlin vector and pEGFP-C1scramble2, otoferlin vector and pEGFP-C1miRNA1, otoferlin vector and pEGFP-C1miRNA2, and otoferlin vector and pEGFP-C1miRNA1 and pEGFP-C1miRNA2. Protein levels as measured from the intensity of the otoferlin bands, normalized to the sample transfected with otoferlin alone, indicating the silencing effect.

transfections and $6 \mu \mathrm{g}$ of DNA in double transfections. Only 2 silencing sequences proved to efficiently knock-down otoferlin (Figure 3.18 blot). The two scramble 
sequences induced a small reduction in the levels of otoferlin, when compared to the sample where only otoferlin was overexpressed. Result of the transfection with otoferlin and the gene-empty vector shows protein levels similar to the samples transfected with the scramble, leading to the conclusion that the reduction is not an effect of the scramble sequence but probably rather due to a reduced uptake of plasmid in multiple-plasmid transfections, resulting in reduced expression levels when co-transfecting with any kind of plasmid. Still, the knock-down of otoferlin caused by the silencing sequence was remarkable no matter what negative control it was compared to (Figure 3.18, graph).

Having successfully designed and selected functional silencing molecules against otoferlin, I proceeded with viral silencing. For the successful delivery of the most prominent miRNA silencing molecules into mouse IHCs, I chose a viral vector strategy, reported to be suitable for the mouse inner ear and causing no immunogenicity (Reisinger et al., 2011; Reuter, 2011). The entire DNA cassette, expressing the EGFP gene and the miRNA, was inserted into an adeno-associated viral (AAV) vector of serotypes 1 and 2 (chimeric capsid). AAV1/2 has previously been shown to effectively transduce IHCs. The expression was driven by the human CMV enhancerchicken beta actin promoter (Figure 3.20 A). The first viral preparations were first tested for their transduction efficiency in HEK cells. Because of the very low titers (AAV-miRNA1: $0.8 \times 10^{8} \mathrm{tu} / \mu \mathrm{l}, \mathrm{AAV}$-miRNA2: $10^{8} \mathrm{tu} / \mu \mathrm{l}$, AAV-scramble: $1.2 \mathrm{x}$ $10^{8} \mathrm{tu} / \mu \mathrm{l}, \mathrm{AAV}$-scramble2: $10^{8} \mathrm{tu} / \mu \mathrm{l}$, ), the expectations for transduction success were rather low. Consequently, the transfection efficiency, as monitored by EGFP fluorescence, was relatively low. Labeling of the cells with DAPI to visualize nuclei did not show any obvious toxicity caused by the viruses (Figure 3.19). 


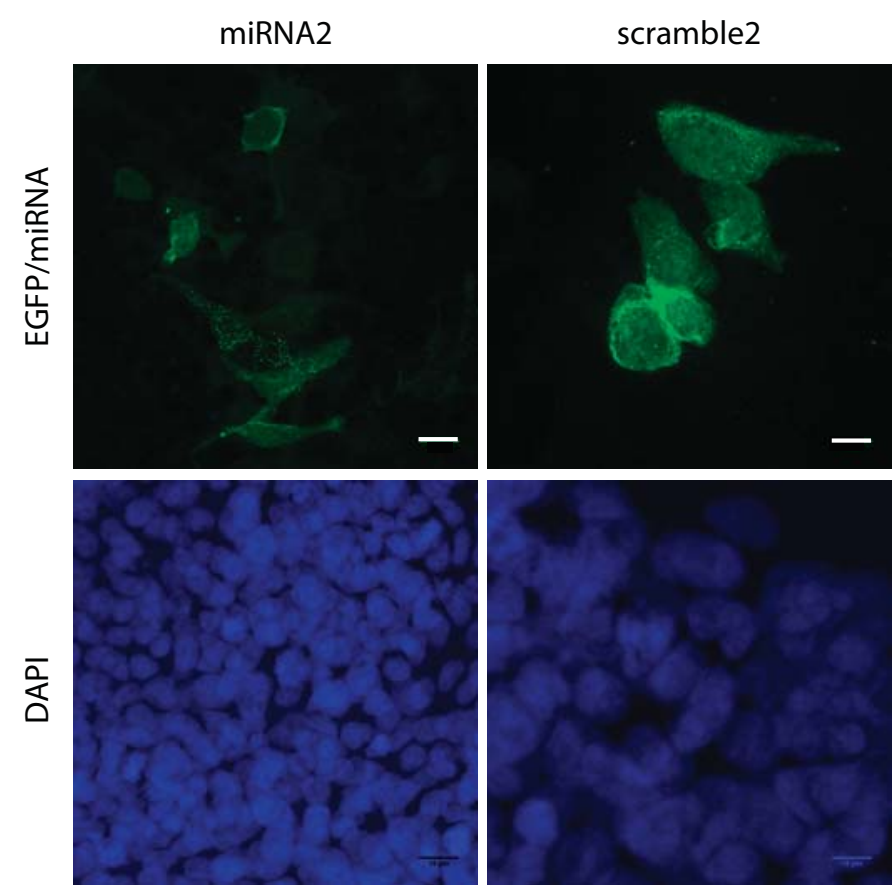

Figure 3.19: In vitro silencing. Confocal images of HEK 293T cells stained with anti-GFP antibody (green) and DAPI (blue) after transfection of the first viral preparation. Examples of transfected cells with pEGFP-C1miRNA2 vector and pEGFP-C1scramble2 vectors. Scale bars are $0.2 \mathrm{~mm}$.

\subsubsection{In vivo silencing}

Transuterine otocyst injections (Reuter, 2011) were performed in order to deliver the virus into the developing otocyst in E11.5 mice. Initially, the first viral preparation, also tested in vitro, were injected. Injections were performed always in the left developing otocyst of each mouse, allowing the use of the organ of Corti of the right side as a negative control. The organs of Corti from the right and left ears of all surviving animals were analyzed at P20 for positive transduction by immunolabeling with an antibody against otoferlin and a GFP antibody conjugated conjugated to Alexa 488. The transduction efficiency in these experiments turned out to be very low $(<5 \%$ transfection rate, Figure $3.20 \mathrm{~B})$. The main reason for this were possibly the low titers of the viruses used here. All right organs of Corti were negative for EGFP (data not shown). A further attempt was made to repeat the injections 
with a second viral preparation. The higher titers of this batch (AAV-miRNA1: $4 \mathrm{x} 10^{8} \mathrm{tu} / \mu \mathrm{l}, \mathrm{AAV}$-scramble1: $\left.4 \times 10^{8} \mathrm{tu} / \mu \mathrm{l}\right)$ proved to be highly advantageous, as the transduction efficiency has drastically increased ( $\sim 40 \%)$. Examples of IHCs from the apical turn of the organ of Corti transduced with miRNA1 and scramble1

A

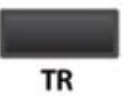

TR

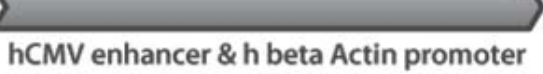

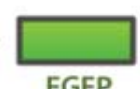

EGFP

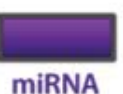

MiRNA

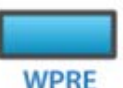

WPRE

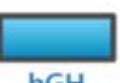

bGH

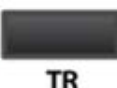

miRNA 1

B
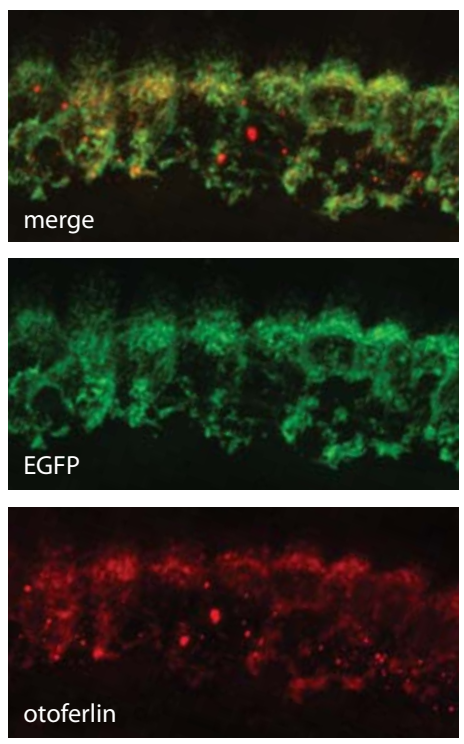

miRNA 2
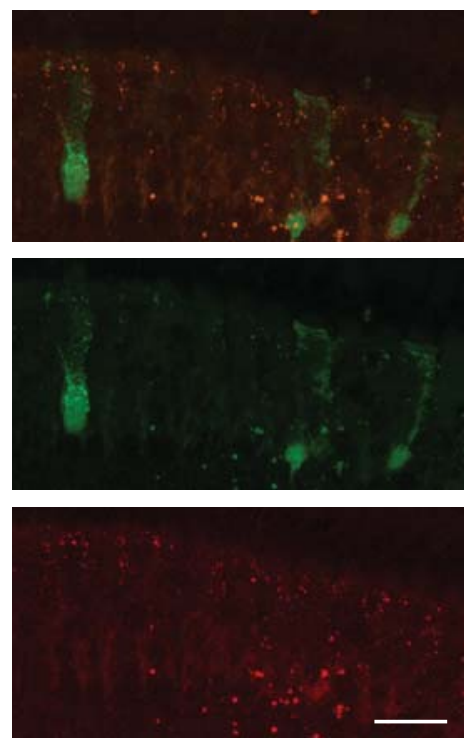

scramble 2
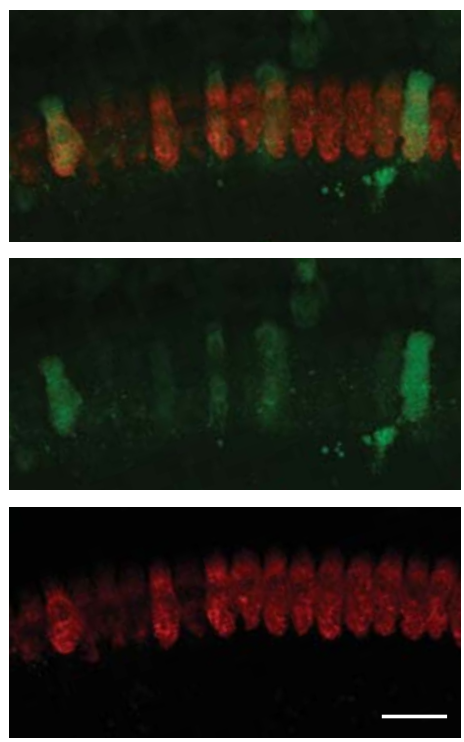

Figure 3.20: Silencing of otoferlin in mouse IHC - First viral preparation. (A) Illustration of the viral sequence after insertion of the "silencing" cassette. (B) Examples of immunolabeled apical left (injected) cochlear turns of P15 CDI animals, injected with the first viral preparation (miRNA1, miRNA2, scramble2). Scale bars are $10 \mu \mathrm{m}$.

are shown in Figures 3.21 and Figures 3.22. Still, the transduction rates were not consistent along the entire length of the organ of Corti and there were variations in the expression levels of EGFP. Nevertheless, in some cases, as shown in the left most panel in Figure 3.21, silencing with miRNA1 almost entirely abolished otoferlin expression. The negative control (non-injected ear, Figure 3.21, right most panel), did not appear to show any changes in the levels of otoferlin. Unfortunately, the results of the injection of the negative scramble sequence were not conclusive. The fluorescence intensity of the otoferlin staining is low in both the right and the left 


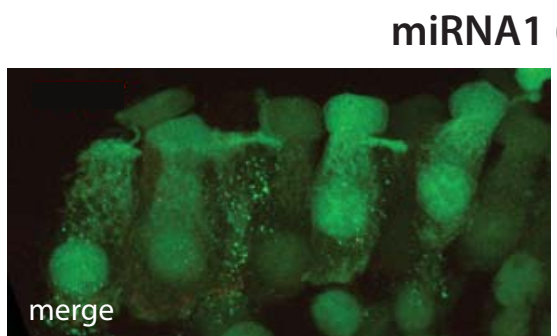

left ear)
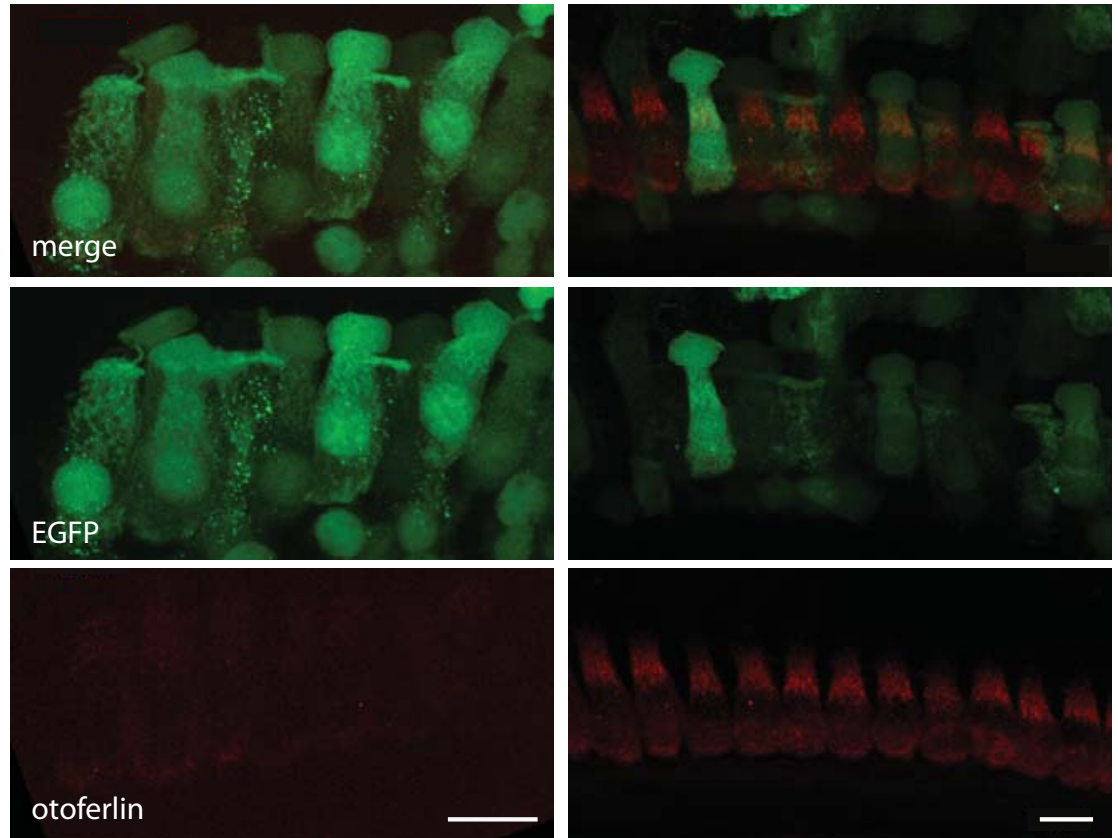

miRNA1 (right ear)
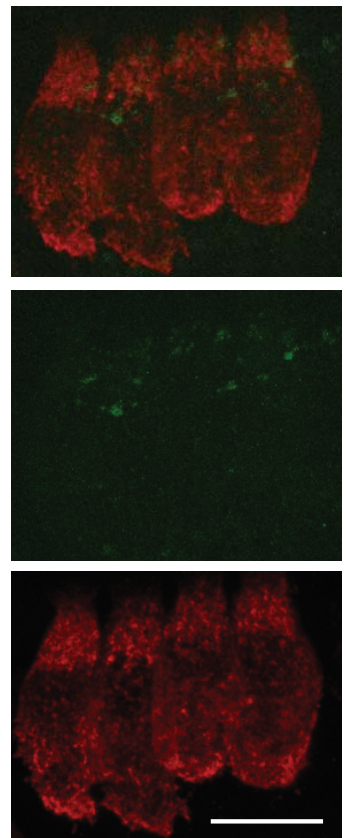

Figure 3.21: Silencing of otoferlin in mouse IHC - Second viral preparation (miRNA1). Examples of immunolabeled apical left (injected) and right (non-injected) cochlear turns of P15 CDI animals, injected with the second viral preparation (miRNA1). Scale bars are $10 \mu \mathrm{m}$.

ear, coming mostly from the presynaptic membrane of the IHCs and suggesting low otoferlin levels. Still, similar fluorescence intensities in both the injected and non-injected ear, suggest that the expression of otoferlin was not impaired, but that the immunohistochemistry did not work properly. Additionally, a similar expression pattern for the protein was observed between scramble1 transduced (EGFP positive) and non-transduced (EGFP negative) IHCs of the injected ears.

In summary, the results suggest that miRNA-mediated silencing of otoferlin in the mouse IHCs of the organ of Corti is feasable, however only when viruses with high titers are used. The strategy followed, seems to yield efficient knock-down, but still needs to be optimized using better negative controls.

\subsubsection{Synaptic transmission of mouse otoferlin heterozygous animals}

As a second approach, to test whether there is a relationship between otoferlin protein levels and synaptic function, I investigated the presynaptic function of 
scramble1 (left ear)
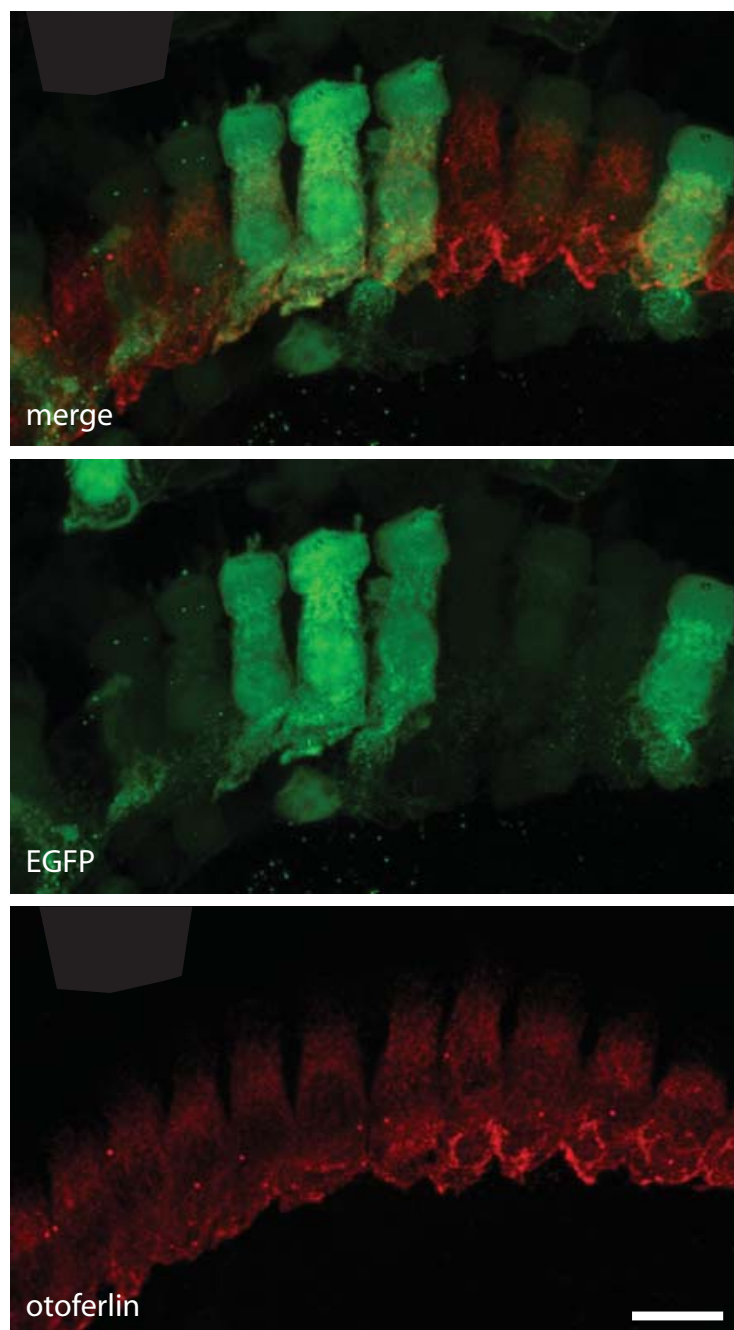

scramble1 (right ear)
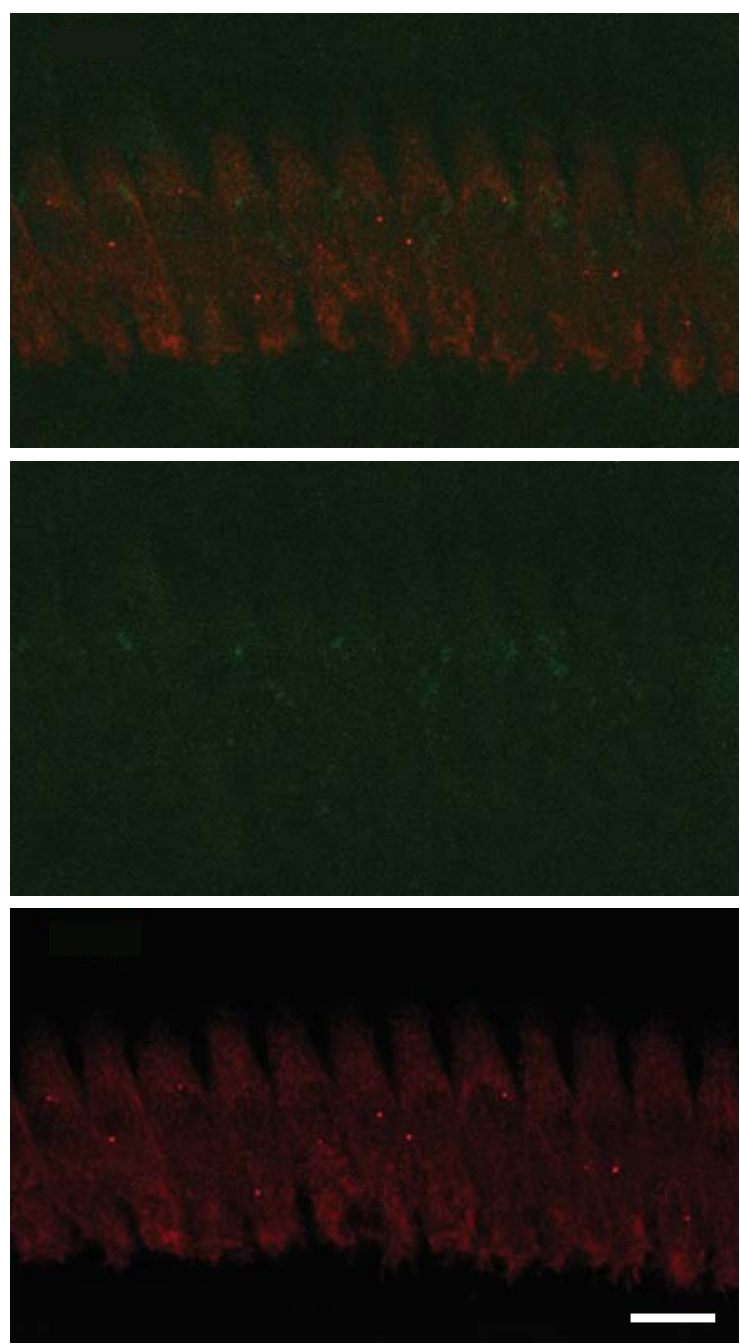

Figure 3.22: Silencing of otoferlin in mouse IHCs - Second viral preparation (scramble1). Examples of immunolabeled apical left (injected) and right (non-injected) cochlear turns of P15 CDI animals, injected with the second viral preparation (scramble1). Scale bars are $10 \mu \mathrm{m}$.

IHCs of the otoferlin heterozygous (Otof ${ }^{+/-}$) animals and littermate controls, using perforated patch-clamp recordings of depolarization-evoked membrane capacitance changes. The results indicate that the ribbon synapse seems to operate normally (Figure 3.23) when otoferlin protein levels are at $70 \%$ of the wild-type levels, with no obvious disturbance in the fast or slow component of exocytosis. If indeed the IHC synaptic function depends on the expressed level of otoferlin, one needs to reduce the expression of otoferlin even further. Perhaps, there is a threshold below which 
A

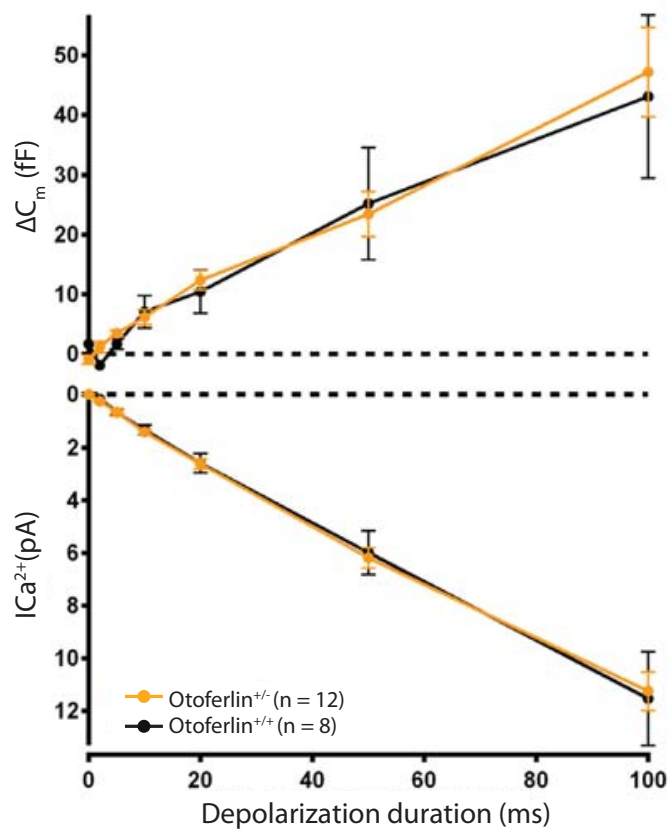

B

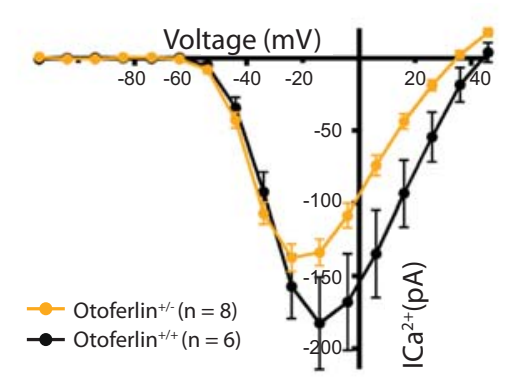

C

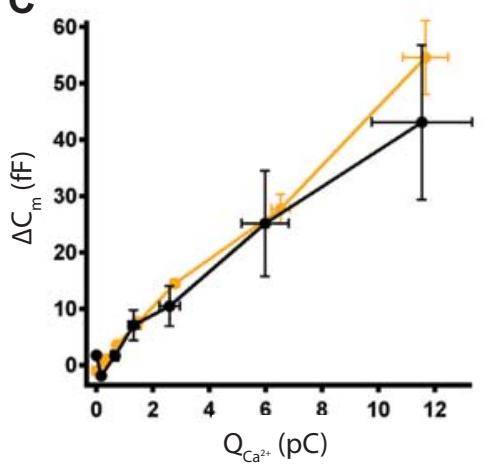

Figure 3.23: Exocytic performance of Otof $^{+/-}$IHCs. (A) Kinetics of exocytosis: Depolarization-evoked membrane capacitance increments $\left(\Delta \mathrm{C}_{m}\right.$ top) and corresponding $\mathrm{Ca}^{2+}$ current integrals (bottom) are plotted versus the duration of depolarization for Otof $^{+/+}($black, $n=8)$ and $\mathrm{Otof}^{+/-}$(yellow, $\mathrm{n}=12$ ) IHCs. (B) Current-voltage (I-V) relationship of IHCs bathed in $2 \mathrm{mM}$ $\left[\mathrm{Ca}^{2+}\right]$. (C) $\Delta \mathrm{C}_{m}$ plotted versus $\mathrm{QCa}^{2+}$. All measurements were performed in IHCs of P14-P17 animals. $\mathrm{n}$ shows the number of the recorded cells. Data points represent mean values \pm s.e.m.

a progressive defect in exocytosis would become obvious. 


\section{4 \\ DisCUSSION}

The inner hair cell (IHC) synapse encodes sound and is the first synapse in the auditory pathway. Ongoing research aims to understand the molecular mechanisms underlying synaptic sound encoding. However many of the constituents of the involved molecular machinery remain unknown. Mice either lacking (KO) otoferlin (Roux et al., 2006) or with $\sim 30 \%$ of the physiological protein levels (pachanga mutant) (Pangršič et al., 2010) show profound deafness as a consequence of a defect in $\mathrm{Ca}^{2+}$-dependent exocytosis. These findings highlighted the importance of otoferlin for the function of in IHC ribbon synapses and led to the hypothesis that it may function as the main $\mathrm{Ca}^{2+}$ sensor (Roux et al., 2006) and/or priming factor at the IHC ribbon synapse (Pangri et al., 2012).

Otoferlin binds to membranes via its C-terminus single transmembrane domain and more specifically, has been found associated with synaptic vesicle membranes and plasma membrane (Roux et al., 2006). The prediction that otoferlin is a tail-anchored (TA) protein (Kalbfleisch et al., 2007), suggests that it may use the TRC40/WRB membrane insertion pathway; nevertheless, to date, there is no biochemical evidence to support this conjecture. The gene that encodes tryptophanrich basic protein (WRB) was originally identified in humans to be linked with a gene for congenital heart disease (CHD) and was proposed to be involved in the pathogenesis of CHD (Egeo et al., 1998). Nevertheless, this hypothesis has not been confirmed and it was only very recently that a role as a TA protein receptor has been attributed to WRB (Vilardi et al., 2011). Additional novel findings demonstrated that mutation of the zebrafish wrb gene (pwi mutant) leads to hearing, visual, and balance deficits (Lin et al., unpublished). However, how exactly WRB, which is a non-synaptic protein, is implicated in hearing, remained to be determined. One 
hypothesis is that the interaction of WRB with otoferlin regulates the membrane insertion of the latter; thus, the absence of WRB influences the function of otoferlin and consequently the synaptic function of IHCs.

This study addressed the relatively novel protein WRB and its function in the mouse auditory system. I used Cre homologous recombination for the targeted deletion of the $W r b$ gene only in those tissues that express glutamate transporter-3 (VGLUT3). Besides IHCs, where only isoform 3 of the transporter family (VGLUT) is expressed (Ruel et al., 2008; Seal et al., 2008), VGLUT3 expression is scattered throughout the glutamatergic synapses in the brain, as well as in cholinergic, serotoninergic, and GABAergic synapses (Fremeau et al., 2001). Additionally, VGLUT3 is expressed in the retina, heart, liver, and kidney (Munguba et al., 2011).

\subsection{Seizures and lethality in the Pwi KO mice}

The first obvious phenotypic characteristic of the Pwi KO mice $\left(\mathrm{Pwi}^{\mathrm{flx} / \mathrm{flx}} \mathrm{VDCR}^{+}\right.$ $\mathrm{CGCT}^{+}$), which was also observed in the zebrafish pwi mutant (Lin et al., submitted), was a circling behavior that was accompanied by a tendency of the mice to lean toward the side. This finding is strongly suggestive of a vestibular disorder that will be investigated in future work. A more dramatic phenotype was the occurence of epileptic seizure-like episodes. In contrast to the seizures described in VGLUT3 $\mathrm{KO}$ mice (Seal et al., 2008), $\mathrm{Pwi}^{\mathrm{fx} / \mathrm{flx}} \mathrm{VDCR}^{+} \mathrm{CGCT}^{+}$mice completely lost motor control and suffered for several minutes during these attacks. There seems to be a neurological disorder underlying this behavior as a consequence of the deletion of the protein in certain brain areas. Usually, seizures occur from a defect in inhibitory neurons, or an imbalance of excitatory-inhibitory input in a part of the brain that leads to over-excitability (Dichter and Ayala, 1987). That is consistent with the idea of a defect related to the excision of the Wrb gene in neurons expressing VGLUT3. In the future, it will be interesting to thoroughly investigate the consequences of the absence of WRB in the mouse brain. One possible hypothesis is that the absence of WRB might perturb the biogenesis of proteins like synaptobrevin 2 , shown to be a TA protein (Kutay et al., 1995) and/or syntaxins, predicted to be TA proteins (Kalbfleisch et al., 2007). If synaptobrevin cannot be inserted properly to the 
ER membrane, it will never get loaded into the synaptic vesicle membrane, which in turn would cause a defect in synaptic exocytosis. Taken that in Pwi KO the Wrb gene gets deleted also in the heart, a neurological disorder in combination with a heart failure problem might result in the rapid deterioration of the condition of $\mathrm{Pwi}^{\mathrm{flx} / \mathrm{flx}} \mathrm{VDCR}^{+} \mathrm{CGCT}^{+}$mice. If this is true, it may indicate the functional importance of the WRB pathway for many TA proteins, which are expressed in the central nervous system and heart. To define the neurological characteristics of $\mathrm{Pwi}^{\mathrm{fl} / \mathrm{flx}} \mathrm{VDCR}^{+} \mathrm{CGCT}^{+}$mice more accurately, future work necessitates the use of electroencephalography to analyze the epileptic seizures.

\subsection{Down-regulation of TRC40 in the absence of WRB}

The consequences of the deletion of the Wrb gene in the Pwi KO mice would be better understood if more light could be shed on the posttranslational protein insertion pathway WRB participates in. Eventually, the identification of interaction partners of WRB within this pathway could help understanding the role of this protein in the various tissues. So far, literature suggests that TRC40 is the protein targeting factor in this pathway (Stefanovic and Hegde, 2007) and interacts with WRB for insertion of tail-anchored (TA) protein into the endoplasmic reticuclum (Vilardi et al., 2011; Yamamoto and Sakisaka, 2012). Interestingly enough, elimination of any of these two proteins in independent mouse models leads to lethality either at embryonic stage (Mukhopadhyay et al., 2006) or within two months of life (this study). Moreover, an attempt to generate a global Wrb KO mouse model failed, because of early embryonic lethality (David Corey, personal communication). In the current study, the expression of TRC40 in IHCs was highly reduced in $\mathrm{Pwi}^{\mathrm{flx} / \mathrm{flx}} \mathrm{VDCR}^{+}$ $\mathrm{CGCT}^{+}$compared to $\mathrm{Pwi}^{\mathrm{flx} / \mathrm{flx}} \mathrm{VDCR}^{-} \mathrm{CGCT}^{+}$. In order to gain more insight into the role of WRB and TRC40, it is crucial to investigate their localization and potential co-localization in IHCs, brain, and other tissue. So far unfortunately, none of the available antibodies for WRB, neither commercial nor custom-made, seem to specifically bind to WRB, at least not in the cochlear tissue. 


\subsection{Progressive hearing loss in Pwi KO mice is probably attributed to the dysfunction of IHCs}

The observed progressive impairment in hearing function upon deletion of Wrb in auditory cells likely results from defects of TA protein trafficking in hair cells. Hearing loss in Pwi KO mice was assessed by studying synaptic neurotransmission at P15 IHCs. The analysis indicated that the IHC synapses of $\mathrm{Pwi}^{\mathrm{flx} / \mathrm{flx}} \mathrm{CRE}^{+} \mathrm{EGFP}^{+}$ mice are able to maintain an intact readily releasable pool (RRP) of vesicles, and promote exocytosis upon $\mathrm{Ca}^{2+}$ influx. The significant decrease in the sustained rate demonstrated that the replenishment process at the ribbon was impaired, which may be responsible for the observed hearing loss. Severely slowed vesicle replenishment at the IHC ribbon synapses has also been reported in the otoferlin pachanga mutant, suggesting that it leads to auditory fatigue and deafness (Pangršič et al., 2010). However, the same assumption cannot be claimed for the control $\mathrm{Pwi}^{\mathrm{flx}} / \mathrm{flx}$ $\mathrm{CRE}^{-} \mathrm{EGFP}^{+}$mice, which showed a significant decrease in both fast and sustained exocytosis, but no hearing deficit. The summating potential, which is thought primarily to indicate the receptor potential of IHCs and outer hair cells (OHCs), was present in the first two age groups studied (groups I and II), while it disappeared approximately at the age when lethality was observed (group III). On the other hand, distortion product oto-acoustic emissions (DPOAE) could be detected in group II. If it can be assumed that the summating potential reflects mostly the sensitivity of IHCs, while DPOAEs report the OHC mechanotransduction activity, then a plausible scenario could be that the hearing deficit is at least partially caused by disruption of mechanotransduction in IHCs. Moreover, a deficit in the transduction machinery has been observed in the zebrafish pwi mutant, suggesting that Wrb in zebrafish modulates the transduction apparatus (Lin et al., unpublished). Therefore, is quite possible that an IHC mechanotransduction defect is also responsible for the observed deafness in Pwi KO mice. To test better this hypothesis a closer investigation of the properties of MET currents and DPOAE recordings at a later age will further help to characterize the hearing phenotype.

From the earliest developmental stage studied, Pwi KO mice had much reduced 
wave I and wave III amplitudes compared to the controls. Wave I is thought to derive from the synchronous spiking activity of the auditory nerve, while wave III is thought to derive from the activity of the neurons in the superior olivary complex in the brainstem (Melcher et al., 1996). The expression of VGLUT3 in the lateral superior olive (Gillespie et al., 2005) is necessary for glutamate release in this area (Noh et al., 2010). Again, it could be assumed that the targeted deletion of the Wrb gene may create several protein imbalances that lead to the dysfunction of sound processing in this auditory neuronal center. Collectively, hearing loss in the pinballwizard $\mathrm{KO}$ could be attributed to a defect in the spiral ganglion neurons, which form the auditory nerve, and possibly also to the superior olivary complex. However, it is unclear why this defect appears to progress in a developmental-dependent manner.

\subsubsection{Recombination in the $W r b$ gene}

The striking finding of perturbed synaptic transmission in $\mathrm{Pwi}^{\mathrm{flx} / \mathrm{flx}} \mathrm{VDCR}^{-} \mathrm{CGCT}^{+}$ mice is difficult to be explained. Apart from synaptic function, no other deficit was observed that could be compared with $\mathrm{Pwi}^{\mathrm{flx} / \mathrm{flx}} \mathrm{VDCR}^{+} \mathrm{CGCT}^{+}$mice. To exclude the possibility of the Wrb gene disruption during the generation of the targeted construct, organs of Corti from P25 $\mathrm{Pwi}^{\mathrm{fx} / \mathrm{flx}} \mathrm{VDCR}^{+} \mathrm{CGCT}^{+}, \mathrm{Pwi}^{\mathrm{flx} / \mathrm{flx}} \mathrm{VDCR}^{-}$ $\mathrm{CGCT}^{+}$, and $\mathrm{Pwi}^{+/+} \mathrm{VDCR}^{+} \mathrm{CGCT}^{+}$animals were analyzed for the length of the Wrb transcript after recombination. Successful recombination in the presence of Cre deleted the exons 2-4 located between the loxP sites, only in $\mathrm{Pwi}^{\text {flx } / \mathrm{flx}} \mathrm{VDCR}^{+}$ $\mathrm{CGCT}^{+}$mice, while in $\mathrm{Pwi}^{\mathrm{flx} / \mathrm{flx}} \mathrm{VDCR}^{-} \mathrm{CGCT}^{+}$, the transcript length is identical to the other control $\left(\mathrm{Pwi}^{+/+} \mathrm{VDCR}^{+} \mathrm{CGCT}^{+}\right)$. Nevertheless, amplifying the region between exons 1 and 2 was not expected to generate a product for $\mathrm{Pwi}^{\mathrm{flx} / \mathrm{flx}} \mathrm{VDCR}^{+}$ $\mathrm{CGCT}^{+}$, assuming that the primer for exon 2 cannot find a binding site inside the recombined Wrb. Interestingly, this was not the case, indicating that part of exon 2 was probably still present in $\mathrm{Pwi}^{\mathrm{flx} / \mathrm{flx}} \mathrm{VDCR}^{+} \mathrm{CGCT}^{+}$. These results lead to two important conclusions. First, they demonstrate that the Wrb gene cannot get deleted in the absence of Cre, as is the case in the $\mathrm{Pwi}^{\mathrm{flx} / \mathrm{flx}} \mathrm{VDCR}^{-} \mathrm{CGCT}^{+}$animals, so the potential undesired excision in these animals can be excluded. Second, the results obtained from the primers targeting the region between exon 1 and 2 propose 
that the first loxP site might be inserted in a different position than the one suggested by the manufacturer of the pinball-wizard conditional mouse line, possibly inside exon2. If this is true then certain structural changes in the gene might affect the resulting mRNA and finally the protein itself. Nevertheless, it should be noted that since the PCR was performed on whole organs of Corti, the observed transcript, most probably appears from those cells where recombination has not happened.

\subsubsection{A defect of the ultrastructure of IHC ribbon synapse}

In the synapse quantification analysis, no significant difference between any three genotypes in age group I was observed. The number of KO ribbon synapses was found to be in the range of that observed in wild-type mice (Meyer et al., 2009). On the contrary, a small decrease was found in age group II, but this finding is not yet conclusive and needs to be investigated further. If there is indeed a progressive decline in the number of synapses at the IHCs of the $\mathrm{Pwi}^{\mathrm{flx} / \mathrm{flx}} \mathrm{VDCR}^{+} \mathrm{CGCT}^{+}$ mice, this could lead to a progressive loss of spiral ganglion neurons, which might explain the progressive hearing dysfunction. In support of this statement, it should be mentioned that at the early age (group I), when cell physiology recordings were performed, the ABR recordings in the $\mathrm{Pwi}^{\mathrm{flx} / \mathrm{flx}} \mathrm{VDCR}^{+} \mathrm{CGCT}^{+}$mice did not indicate a complete hearing impairment.

To gain more insight into the mechanism of the slowed replenishment at the pinballwizard $\mathrm{KO}$ IHC ribbon synapse, electron microscopy (EM) was used to examine the structure and formation of ribbon synapses. In the two genotypes studied, the average number of ribbon-associated synaptic vesicles per synapse section was significantly reduced in IHCs of $\mathrm{Pwi}^{\mathrm{flx} / \mathrm{flx}} \mathrm{VDCR}^{+} \mathrm{CGCT}^{+}$mice compared to $\mathrm{Pwi}^{\mathrm{fx} / \mathrm{flx}}$ $\mathrm{VDCR}^{-} \mathrm{CGCT}^{+}$mice, which interestingly, had almost exactly the same number of vesicles as the stimulated synapse of the pachanga mutant (Pangršič et al., 2010). However, ribbon formation did not appear to be significantly impaired and can therefore probably not explain the reduced number of tethered vesicles. It is likely that the probability that vesicles could accumulate around the ribbon was diminished because of a significant increase in unusually large vesicles, which formed clusters either in the proximity of the ribbon or in an $500 \mathrm{~nm}$ area around it. The ex- 
act nature of such structures is unknown and should be further investigated with immuno-EM using endosomal markers, e.g., Rab5 and EEA1, or synaptic vesicle markers, e.g., synaptobrevin 2 and otoferlin.

The identification of a synaptic link between the absence of WRB and the observed hearing defects was intriguing, and my working hypothesis was that the disruption of the membrane insertion pathway could influence the trafficking of otoferlin from the cytoplasmic sites of translation to the ER membranes. If otoferlin cannot get incorporated to the ER membrane, it will not be folded correctly and will not be present on the vesicles. Unfortunately, I was unable to investigate this hypothesis during the course of this study, which will require future in vitro biochemical experiments. However, in order to gain some insight into this hypothesis, I evaluated the protein levels of otoferlin using a semi-quantitative approach. I observed a tendency for $\mathrm{Pwi}^{\mathrm{flx} / \mathrm{flx}} \mathrm{VDCR}^{+} \mathrm{CGCT}^{+}$mice to express less otoferlin at the apex and base of IHCs than control $\mathrm{Pwi}^{+/+} \mathrm{VDCR}^{+} \mathrm{CGCT}^{+} \mathrm{IHCs}$, whereas for $\mathrm{Pwi}^{\mathrm{flx} / \mathrm{flx}}$ VDCR $^{-} \mathrm{CGCT}^{+}$IHCs, the opposite trend was observed. In addition, a distribution pattern analysis indicated a mostly diffuse distribution of otoferlin throughout the cytoplasm. However, these results are preliminary and no firm conclusions can be made at this point. A more systematic analysis should be conducted in the future. For the absolute quantification of protein levels, western blot analysis of the whole organs of Corti would be beneficial. Unfortunately, none of the efforts yielded any result, mainly because of the small size of the tissue and the requirement of relatively large amounts of material for successful western blot analysis.

\subsection{Hypotheses on the function of WRB in IHCs}

The deletion of the Wrb from IHCs apparently creates an exocytic deficit, which is manifested in in $\mathrm{Pwi}^{\mathrm{flx} / \mathrm{flx}} \mathrm{CRE}^{+} \mathrm{EGFP}^{+}$mice as a reduced replenishment rate and large vesicle clusters. The proposed hypothesis is that in the absence of WRB, trafficking of certain synaptic protein components or even ribbon scaffold proteins gets disrupted. Thus, the observed phenotype could be attributed to the dysfunction of several proteins at the synapse. If synaptic vesicles cannot carry all required proteins, e.g., otoferlin, or even bud from the ER, they will never have the opportunity 
to reach the synapse and become fusion-competent, but instead they might form clusters. On the other hand, a compensatory mechanism might still enable the partial function of the membrane insertion pathway. It was recently shown (Yamamoto and Sakisaka, 2012) in mammalian cell-based in vitro assays, that calcium-signaling modulating cyclophilin ligand (CAML) is part of the receptor complex of TA proteins in the ER. Both CAML and WRB are required for TRC40/TA protein binding to the ER, but they also compete for this interaction. Therefore, it is tempting to suggest that in the absence of WRB, CAML takes over the insertion of proteins into the ER; however, it is unable to rescue the process completely. The tubular structures that were observed close to the presynaptic membrane of the $\mathrm{Pwi}^{\mathrm{flx} / \mathrm{flx}} \mathrm{CRE}^{+}$ $\mathrm{EGFP}^{+} \mathrm{IHCs}$ were considered to be ER membranes with ribosomes. Although this notion needs to be further confirmed, the presence of the ER so close to the ribbon synapse might also serve as a compensatory mechanism to the high demands for protein trafficking. Again, the identification of the distribution pattern of WRB inside IHCs is crucial for confirming its role as a TA protein receptor and also to indicate its interaction partners.

Considering these findings together, the Pwi KO mice share many similarities with the otoferlin pachanga mutant. In both, the fast component of synaptic vesicle release is intact, but vesicle replenishment is compromised, indicated by decreased sustained rates of exocytosis. While in pachanga mutant sustained rate of release was decreased by $\sim 70 \%$, in pinball-wizard $\mathrm{KO}$ it was reduced by $\sim 50 \%$. In pachanga mutant one single point mutation of otoferlin caused the inability of the IHC synapse to refill with synaptic vesicles and maintain neurotransmission, while in pinball-wizard $\mathrm{KO}$, deletion of WRB may disrupt the membrane protein insertion pathway and influence the biogenesis of multiple other proteins with a role at the synapse. If a progressive reduction in the number of synapses can be shown, it would suggest the downstream degeneration of spiral ganglion neurons and provide a second possible explanation of the absence of wave I in ABR recordings. Together with or independent of an impaired transduction machinery in OHCs it would suggest the early disruption of the auditory pathway function.

The choice of the Vglut3-CRE reporter line VDCR-CGCT was based on the 
desire of IHC-specific Wrb deletion. Apparently, targeted excision of the gene did not occurr solely in the cochlea, given the broad tissue expression of VGLUT3. Although the physiological findings are of great experimental interest and reveal a major role of WRB in multiple tissues, it is not easy to isolate one particular defect, excluding the implication of others. Alternatively, another IHC-specific Cre mouse line could be used in order to study the function of WRB exclusively in the auditory pathway, which however, is currently not available yet.

\subsection{Silencing otoferlin in the mammalian inner ear}

A parallel project that was conducted, aimed to establish a strategy for miRNAmediated gene silencing. The motivation was guided by the idea, initially suggested by Pangršič et al., (2010), that perturbations in the protein levels of otoferlin, result in variations of the synaptic performance at the IHC ribbon synapse. It was thus tempting, to try to manipulate the protein levels and investigate the phenotypic variations. The choice of miRNA silencing cassettes and not of shRNA or siRNA, was based on two factors. First, I needed to achieve stable gene silencing (Brummelkamp et al., 2002; Paddison et al., 2002), which could not be the case with siRNA. Second, I wanted that the silencing molecule would mimic as much as possible the endogenous mammalian RNA interference (RNAi) pathway, in order to minimize the chances of cellular toxicity, due to off-target effects. The use of shRNA molecules was avoided, because they require special promoters (Liu and Berkhout, 2011) as they are transcribed by RNA polymerase III, whereas miRNA is transcribed as any other gene by RNA polymerase II. Therefore, it was advantageous to use miRNA and consequently only one promoter to drive simultaneously the expression of an miRNA silencing molecule and of the reporter gene. The successful in vitro experiments, confirmed the designed strategy and indicated those miRNA silencing molecules that significantly could reduce the levels of otoferlin as compared to controls. Taking full advantage of the recently established method of embryonic trans-uterine otocyst injection (Reuter, 2011), miRNA silencing cassettes were futher delivered by adeno-associated viral (AAV) vectors to the developing otocyst of 11.5 days embryos. The chimeric AAV1/2 serotype has been shown to effectively 
transduce mouse IHCs (Reuter, 2011), while other viruses, like Semliki, Forest Virus, Lentivirus and several AAV serotypes have failed to do so (Reisinger et al., 2011). Many other attempts have achieved the in vivo delivery of viruses in the mouse cochlea, mostly through cochleostomy and round window membrane (reviewed in Maeda et al., 2009).

The application of silencing in vivo was not a straightforward process. Multiple viral preparations had to be tested first in vitro and then in vivo, since the titer was a critical factor for the transduction efficiency. With the first viral preparation, there was almost no transduction in the organ of Corti, but only some scattered positive supporting cells and rarely IHCs. In those cases the results were not conclusive. The results of the second viral preparation, were drastically different. The viruses were able to transduce IHCs and OHCs with a good indication of otoferlin silencing in IHCs. Unfortunately, the long process of the experimental design, the in vitro evaluation, and viral preparation, did not permit the extensive analysis of many injected animals and the proper evaluation of silencing. It is still questionable whether silencing was successful at all and if yes, then to what extent. More systematic analysis by immunolabeling and quantification of otoferlin protein levels is desirable. Cell physiology experiments at organs of Corti of animals injected with viruses carrying miRNA silencing molecules will additionally contribute to a more thorough analysis of the synaptic function after silencing. Finally, testing for hearing function could provide evidence for a potential silencing-evoked deficit.

The use of miRNA-like silencing molecules, delivered by AAV into the mouse developing otocyst, is a novel approach to knock-down selected IHC genes and abolish protein translation. The advantage of this approach is that in some cases it could possibly substitute the need for generation of a transgenic mouse model. While this is a newly method, in the future, optimization is intended and if successful, it could be applied for modifying the levels of any desired protein. For example, in the mouse IHC ribbon synapse, the role of ribbon and most of its structural components is not yet clear. The silencing of RIBEYE, the most abundant, ribbon-specific protein, would allow a more thorough investigation of the role of ribbon in the formation 
and function of the synapse.

In parallel, I studied the synaptic transmission of the otoferlin heterozygous $\left(\right.$ Otof $\left.^{+/-}\right)$apical IHCs, which also show a modest reduction in the expression level of otoferlin in IHCs (Pangršič et al., 2010). It was further shown that in these mice the amplitude of wave I was almost the half of the amplitude in the control littermates. These two facts intrigued me to test whether the IHC ribbon synapse can operate with $70 \%$ remaining otoferlin levels. I found normal $\mathrm{Ca}^{2+}$-dependent exocytosis, which obviously indicates that the remaining protein levels suffice for normal synaptic function. The functional differences in three otoferlin mouse mutants, Otof ${ }^{-/-}$(Roux et al., 2006), pachanga (Pangršič et al., 2010), and Otof ${ }^{+/-}$(not yet fully characterized), indicate that otoferlin has rather a "flexible" mode of function. Variation in the expression levels, directs the synapse to adjust its function. From this point of view, it remains to be elucidated what is the minimum level of protein in order for the synapse to be fully functional. 



\section{BIBLIOGRAPHY}

Abell, B.M., Pool, M.R., Schlenker, O., Sinning, I., and High, S.: Signal recognition particle mediates post-translational targeting in eukaryotes. EMBO J 23 (14): 2755-2764, 2004. PMID: 15229647 PMCID: PMC514945.

Abell, B.M., Rabu, C., Leznicki, P., Young, J.C., and High, S.: Post-translational integration of tail-anchored proteins is facilitated by defined molecular chaperones. J Cell Sci 120 (10): 1743-1751, 2007.

Achanzar, W.E. and Ward, S.: A nematode gene required for sperm vesicle fusion. J Cell Sci 110 (9): 1073-1081, 1997.

Ashmore, J.: Cochlear outer hair cell motility. Physiol Rev 88 (1): 173-210, 2008.

Bansal, D., Miyake, K., Vogel, S.S., Groh, S., Chen, C.C., Williamson, R., McNeil, P.L., and Campbell, K.P.: Defective membrane repair in dysferlin-deficient muscular dystrophy. Nature 423 (6936): 168-172, 2003. PMID: 12736685.

Bernatchez, P.N., Sharma, A., Kodaman, P., and Sessa, W.C.: Myoferlin is critical for endocytosis in endothelial cells. Am J Physiol Cell Physiol 297 (3): C484C492, 2009.

Beurg, M., Michalski, N., Safieddine, S., Bouleau, Y., Schneggenburger, R., Chapman, E.R., Petit, C., and Dulon, D.: Control of exocytosis by synaptotagmins and otoferlin in auditory hair cells. J. Neurosci 30 (40): 13.281-13.290, 2010. PMID: 20926654.

Beutner, D. and Moser, T.: The presynaptic function of mouse cochlear inner hair cells during development of hearing. J Neurosci 21 (13): 4593, 2001. 
Borgese, N., Gazzoni, I., Barberi, M., Colombo, S., and Pedrazzini, E.: Targeting of a tail-anchored protein to endoplasmic reticulum and mitochondrial outer membrane by independent but competing pathways. Mol. Biol. Cell 12 (8): 2482-2496, 2001. PMID: 11514630.

Brandt, A., Striessnig, J., and Moser, T.: CaV1. 3 channels are essential for development and presynaptic activity of cochlear inner hair cells. J Neurosci 23 (34): 10.832-10.840, 2003.

Caplen, N.J., Parrish, S., Imani, F., Fire, A., and Morgan, R.A.: Specific inhibition of gene expression by small double-stranded RNAs in invertebrate and vertebrate systems. Proc Natl Acad Sci U S A 98 (17): 9742-9747, 2001. PMID: 11481446 PMCID: PMC55523.

Covian-Nares, J.F., Koushik, S.V., Puhl, H.L., and Vogel, S.S.: Membrane wounding triggers ATP release and dysferlin-mediated intercellular calcium signaling. J Cell Sci 123 (11): 1884-1893, 2010.

Dallos, P.: The active cochlea. J. Neurosci. 12 (12): 4575-4585, 1992.

Dallos, P.: Cochlear amplification, outer hair cells and prestin. Curr Opin Neurobiol 18 (4): 370-376, 2008. PMID: 18809494 PMCID: PMC2630119.

Deng, L., Kaeser, P.S., Xu, W., and Sdhof, T.C.: RIM proteins activate vesicle priming by reversing autoinhibitory homodimerization of munc13 69 (2): 317$331,2011$.

tom Dieck, S., Altrock, W.D., Kessels, M.M., Qualmann, B., Regus, H., Brauner, D., Fejtov, A., Bracko, O., Gundelfinger, E.D., and Brandsttter, J.H.: Molecular dissection of the photoreceptor ribbon synapse: physical interaction of bassoon and RIBEYE is essential for the assembly of the ribbon complex. J Cell Biol 168 (5): 825-836, 2005. PMID: 15728193.

Doherty, K.R., Cave, A., Davis, D.B., Delmonte, A.J., Posey, A., Earley, J.U., Hadhazy, M., and McNally, E.M.: Normal myoblast fusion requires myoferlin. Development 132 (24): 5565-5575, 2005. PMID: 16280346. 
Dulon, D., Safieddine, S., Jones, S.M., and Petit, C.: Otoferlin is critical for a highly sensitive and linear calcium-dependent exocytosis at vestibular hair cell ribbon synapses 29 (34): 10.474-10.487, 2009.

Egeo, A., Mazzocco, M., Sotgia, F., Arrigo, P., Oliva, R., Bergonn, S., Nizetic, D., Rasore-Quartino, A., and Scartezzini, P.: Identification and characterization of a new human cDNA from chromosome 21q22.3 encoding a basic nuclear protein. Hum Genet 102 (3): 289-293, 1998.

Elbashir, S.M., Harborth, J., Lendeckel, W., Yalcin, A., Weber, K., and Tuschl, T.: Duplexes of 21-nucleotide RNAs mediate RNA interference in cultured mammalian cells. Nature 411 (6836): 494-498, 2001.

Favaloro, V., Vilardi, F., Schlecht, R., Mayer, M.P., and Dobberstein, B.: Asna1/TRC40-mediated membrane insertion of tail-anchored proteins. J Cell Sci 123 (9): 1522-1530, 2010. PMID: 20375064.

Frank, T., Rutherford, M.A., Strenzke, N., Neef, A., Pangri, T., Khimich, D., Fejtova, A., Gundelfinger, E.D., Liberman, M.C., Harke, B., Bryan, K.E., Lee, A., and Egner: Bassoon and the synaptic ribbon organize ca+ channels and vesicles to add release sites and promote refilling. Neuron 68 (4): 724-738, 2010.

Fremeau, R.T., Troyer, M.D., Pahner, I., Nygaard, G.O., Tran, C.H., Reimer, R.J., Bellocchio, E.E., Fortin, D., Storm-Mathisen, J., and Edwards, R.H.: The expression of vesicular glutamate transporters defines two classes of excitatory synapse 31 (2): 247-260, 2001.

Gebhart, M., Juhasz-Vedres, G., Zuccotti, A., Brandt, N., Engel, J., Trockenbacher, A., Kaur, G., Obermair, G.J., Knipper, M., Koschak, A., and Striessnig, J.: Modulation of cav1.3 ca2+ channel gating by rab3 interacting molecule 44 (3): 246-259, 2010.

Gerges, N.Z., Backos, D.S., and Esteban, J.A.: Local control of AMPA receptor trafficking at the postsynaptic terminal by a small GTPase of the rab family. $J$. Biol. Chem. 279 (42): 43.870-43.878, 2004. 
Glowatzki, E. and Fuchs, P.A.: Transmitter release at the hair cell ribbon synapse. Nat Neurosci 5 (2): 147-154, 2002. PMID: 11802170.

Gross, J.M., Perkins, B.D., Amsterdam, A., Egana, A., Darland, T., Matsui, J.I., Sciascia, S., Hopkins, N., and Dowling, J.E.: Identification of zebrafish insertional mutants with defects in visual system development and function. Genetics 170 (1): 245-261, 2005. PMID: 15716491 PMCID: PMC1444939.

Hegde, R.S. and Keenan, R.J.: Tail-anchored membrane protein insertion into the endoplasmic reticulum 2011.

Heidrych, P., Zimmermann, U., Bre, A., Pusch, C.M., Ruth, P., Pfister, M., Knipper, M., and Blin, N.: Rab8b GTPase, a protein transport regulator, is an interacting partner of otoferlin, defective in a human autosomal recessive deafness form. Hum. Mol. Genet. 17 (23): 3814-3821, 2008.

Heidrych, P., Zimmermann, U., Kuhn, S., Franz, C., Engel, J., Duncker, S.V., Hirt, B., Pusch, C.M., Ruth, P., Pfister, M., Marcotti, W., Blin, N., and Knipper, M.: Otoferlin interacts with myosin VI: implications for maintenance of the basolateral synaptic structure of the inner hair cell. Hum. Mol. Genet. 18 (15): 2779-2790, 2009 .

Helfmann, S., Neumann, P., Tittmann, K., Moser, T., Ficner, R., and Reisinger, E.: The crystal structure of the CA domain of otoferlin reveals an unconventional top loop region. J. Mol. Biol 406 (3): 479-490, 2011. PMID: 21216247.

Jantti, J., Keranen, S., Toikkanen, J., Kuismanen, E., Ehnholm, C., Soderlund, H., and Olkkonen, V.M.: Membrane insertion and intracellular transport of yeast syntaxin sso2p in mammalian cells. J Cell Sci 107 (12): 3623-3633, 1994.

Johnson, C.P. and Chapman, E.R.: Otoferlin is a calcium sensor that directly regulates SNARE-mediated membrane fusion. J Cell Biol 191 (1): 187-197, 2010. PMID: 20921140 PMCID: PMC2953437.

Johnson, S.L., Franz, C., Kuhn, S., Furness, D.N., Ruttiger, L., Munkner, S., Rivolta, M.N., Seward, E.P., Herschman, H.R., Engel, J., Knipper, M., and Mar- 
cotti, W.: Synaptotagmin IV determines the linear ca2+ dependence of vesicle fusion at auditory ribbon synapses. Nat Neurosci 13 (1): 45-52, 2010. PMID: 20010821 PMCID: PMC3390740.

Joseph Sambrook and David W. Russell: Molecular Cloning: A Laboratory Manual (2nd Edition). Cold Spring Harbor Laboratory Press, New York, third edition edition, 1989.

Kalbfleisch, T., Cambon, A., and Wattenberg, B.W.: A bioinformatics approach to identifying tail-anchored proteins in the human genome 8 (12): 16871694, 2007.

Khimich, D., Nouvian, R., Pujol, R., tom Dieck, S., Egner, A., Gundelfinger, E.D., and Moser, T.: Hair cell synaptic ribbons are essential for synchronous auditory signalling. Nature 434 (7035): 889-894, 2005.

Kuroda, R., Ikenoue, T., Honsho, M., Tsujimoto, S., Mitoma, J.y., and Ito, A.: Charged amino acids at the carboxyl-terminal portions determine the intracellular locations of two isoforms of cytochromeb 5. J. Biol. Chem. 273 (47): 31.09731.102, 1998.

Kutay, U., Ahnert-Hilger, G., Hartmann, E., Wiedenmann, B., and Rapoport, T.A.: Transport route for synaptobrevin via a novel pathway of insertion into the endoplasmic reticulum membrane. EMBO J 14 (2): 217-223, 1995. PMID: 7835332 PMCID: PMC398073.

Kutay, U., Hartmann, E., and Rapoport, T.A.: A class of membrane proteins with a c-terminal anchor. Trends in Cell Biology 3 (3): 72-75, 1993.

Lek, A., Evesson, F.J., Sutton, R.B., North, K.N., and Cooper, S.T.: Ferlins: Regulators of vesicle fusion for auditory neurotransmission, receptor trafficking and membrane repair 13 (2): 185194, 2012.

Lenzi, D., Runyeon, J.W., Crum, J., Ellisman, M.H., and Roberts, W.M.: Synaptic vesicle populations in saccular hair cells reconstructed by electron tomography. $J$. Neurosci. 19 (1): 119-132, 1999. 
Liberman, M.: Single-neuron labeling in the cat auditory nerve 216 (4551): 1239 $-1241,1982$.

Linstedt, A.D., Foguet, M., Renz, M., Seelig, H.P., Glick, B.S., and Hauri, H.P.: A c-terminally-anchored golgi protein is inserted into the endoplasmic reticulum and then transported to the golgi apparatus. Proc Natl Acad Sci U S A 92 (11): 5102-5105, 1995. PMID: 7761455 PMCID: PMC41856.

Liu, Y.P. and Berkhout, B.: miRNA cassettes in viral vectors: Problems and solutions. Biochimica et Biophysica Acta (BBA) - Gene Regulatory Mechanisms 1809 (1112): 732-745, 2011.

LoGiudice, L., Sterling, P., and Matthews, G.: Mobility and turnover of vesicles at the synaptic ribbon 28 (12): 3150, 2008.

Manley, G.A.: Cochlear mechanisms from a phylogenetic viewpoint. PNAS 97 (22): 11.736-11.743, 2000.

Mariappan, M., Li, X., Stefanovic, S., Sharma, A., Mateja, A., Keenan, R.J., and Hegde, R.S.: A ribosome-associating factor chaperones tail-anchored membrane proteins. Nature 466 (7310): 1120-1124, 2010. PMID: 20676083 PMCID: PMC2928861.

Melcher, J.R., Guinan Jr, J.H., Knudson, I.M., and Kiang, N.Y.: Generators of the brainstem auditory evoked potential in cat II. correlating lesion sites with waveform changes 93 (1-2): 28-51, 1996.

Merchan-Perez, A. and Liberman, M.C.: Ultrastructural differences among afferent synapses on cochlear hair cells: correlations with spontaneous discharge rate. $J$. Comp. Neurol 371 (2): 208-221, 1996. PMID: 8835727.

Meyer, A.C., Frank, T., Khimich, D., Hoch, G., Riedel, D., Chapochnikov, N.M., Yarin, Y.M., Harke, B., Hell, S.W., Egner, A., and Moser, T.: Tuning of synapse number, structure and function in the cochlea. Nat Neurosci 12 (4): 444-453, 2009 . 
Meyer, A.C. and Moser, T.: Structure and function of cochlear afferent innervation. Curr Opin Otolaryngol Head Neck Surg 18 (5): 441-446, 2010. PMID: 20802334.

Montgomery, M.K. and Fire, A.: Double-stranded RNA as a mediator in sequencespecific genetic silencing and co-suppression. Trends in Genetics 14 (7): 255-258, 1998.

Moser, T. and Beutner, D.: Kinetics of exocytosis and endocytosis at the cochlear inner hair cell afferent synapse of the mouse. Proc Natl Acad Sci U S A 97 (2): 883-888, 2000. PMID: 10639174 PMCID: PMC15425.

Moser, T., Brandt, A., and Lysakowski, A.: Hair cell ribbon synapses. Cell Tissue Res 326 (2): 347-359, 2006.

Munguba, G.C., Camp, A.S., Risco, M., Tapia, M.L., Bhattacharya, S.K., and Lee, R.K.: Vesicular glutamate transporter 3 in age-dependent optic neuropathy. Mol Vis 17: 413-419, 2011. PMID: 21311743 PMCID: PMC3036565.

Murata, K., Degmetich, S., Kinoshita, M., and Shimada, E.: Expression of the congenital heart disease 5/tryptophan rich basic protein homologue gene during heart development in medaka fish, oryzias latipes 51 (2): 95107, 2009.

Neef, A., Khimich, D., Pirih, P., Riedel, D., Wolf, F., and Moser, T.: Probing the mechanism of exocytosis at the hair cell ribbon synapse 27 (47): 12.933-12.944, 2007.

Nouvian, R., Beutner, D., Parsons, T., and Moser, T.: Structure and function of the hair cell ribbon synapse. J Membrane Biol 209 (2-3): 153-165, 2006.

Nouvian, R., Neef, J., Bulankina, A.V., Reisinger, E., Pangri, T., Frank, T., Sikorra, S., Brose, N., Binz, T., and Moser, T.: Exocytosis at the hair cell ribbon synapse apparently operates without neuronal SNARE proteins. Nat Neurosci 14 (4): 411-413, 2011.

Ohara-Imaizumi, M., Ohtsuka, T., Matsushima, S., Akimoto, Y., Nishiwaki, C., Nakamichi, Y., Kikuta, T., Nagai, S., Kawakami, H., Watanabe, T., and Nagamatsu, S.: ELKS, a protein structurally related to the active zone-associated 
protein CAST, is expressed in pancreatic cells and functions in insulin exocytosis: Interaction of ELKS with exocytotic machinery analyzed by total internal reflection fluorescence microscopy 16 (7): 3289-3300, 2005.

Pangri, T., Reisinger, E., and Moser, T.: Otoferlin: a multi-c2 domain protein essential for hearing. Trends Neurosci. 35 (11): 671-680, 2012. PMID: 22959777.

Parsons, T.D. and Sterling, P.: Synaptic ribbon 37 (3): 379-382, 2003.

Pedrazzini, E., Villa, A., and Borgese, N.: A mutant cytochrome b5 with a lengthened membrane anchor escapes from the endoplasmic reticulum and reaches the plasma membrane. Proc Natl Acad Sci U S A 93 (9): 4207-4212, 1996. PMID: 8633042 PMCID: PMC39513.

Peter Dallos, Arthur N. Popper, and Richard R. Fay: The Cochlea, volume 8 of $I V$. Springer Verlag, New York, Inc, 1996.

Platzer, J., Engel, J., Schrott-Fischer, A., Stephan, K., Bova, S., Chen, H., Zheng, H., and Striessnig, J.: Congenital deafness and sinoatrial node dysfunction in mice lacking class d l-type ca2+ channels. Cell 102 (1): 89-97, 2000. PMID: 10929716.

Rabu, C., Schmid, V., Schwappach, B., and High, S.: Biogenesis of tail-anchored proteins: the beginning for the end? J. Cell. Sci 122 (20): 3605-3612, 2009. PMID: 19812306.

Rabu, C., Wipf, P., Brodsky, J.L., and High, S.: A precursor-specific role for Hsp40/Hsc70 during tail-anchored protein integration at the endoplasmic reticulum. J. Biol. Chem. 283 (41): 27.504-27.513, 2008.

Ramakrishnan, N.A., Drescher, M.J., and Drescher, D.G.: Direct interaction of otoferlin with syntaxin 1A, SNAP-25, and the l-type voltage-gated calcium channel cav1.3. J. Biol. Chem 284 (3): 1364-1372, 2009. PMID: 19004828.

Reisinger, E., Bresee, C., Neef, J., Nair, R., Reuter, K., Bulankina, A., Nouvian, R., Koch, M., Bckers, J., Kastrup, L., Roux, I., Petit, C., Hell, S.W., Brose, N., Rhee, J.S., Kgler, S., Brigande, J.V., and Moser, T.: Probing the functional equivalence 
of otoferlin and synaptotagmin 1 in exocytosis. J. Neurosci. 31 (13): 4886-4895, 2011. PMID: 21451027.

Reuter, K.: Biochemistry and physiological role of otoferlin. $\mathrm{PhD}$ thesis, University of Gttingen, 2011.

Rodrguez-Ballesteros, M., Reynoso, R., Olarte, M., Villamar, M., Morera, C., Santarelli, R., Arslan, E., Med, C., Curet, C., Vlter, C., Sainz-Quevedo, M., Castorina, P., Ambrosetti, U., Berrettini, S., Frei, K., Tedn, S., Smith, J., Cruz Tapia, M., Cavall, L., Gelvez, N., Primignani, P., Gmez-Rosas, E., Martn, M., MorenoPelayo, M.A., Tamayo, M., Moreno-Barral, J., Moreno, F., and del Castillo, I.: A multicenter study on the prevalence and spectrum of mutations in the otoferlin gene (OTOF) in subjects with nonsyndromic hearing impairment and auditory neuropathy. Hum. Mutat. 29 (6): 823-831, 2008. PMID: 18381613.

Roux, I., Safieddine, S., Nouvian, R., Grati, M., Simmler, M.C., Bahloul, A., Perfettini, I., Le Gall, M., Rostaing, P., Hamard, G., Triller, A., Avan, P., Moser, T., and Petit, C.: Otoferlin, defective in a human deafness form, is essential for exocytosis at the auditory ribbon synapse. Cell 127 (2): 277-289, 2006. PMID: 17055430.

Ruel, J., Emery, S., Nouvian, R., Bersot, T., Amilhon, B., Van Rybroek, J.M., Rebillard, G., Lenoir, M., Eybalin, M., Delprat, B., Sivakumaran, T.A., Giros, B., El Mestikawy, S., Moser, T., Smith, R.J., Lesperance, M.M., and Puel, J.L.: Impairment of SLC17A8 encoding vesicular glutamate transporter-3, VGLUT3, underlies nonsyndromic deafness DFNA25 and inner hair cell dysfunction in null mice. Am J Hum Genet 83 (2): 278-292, 2008. PMID: 18674745 PMCID: PMC2495073.

Rutherford, M.A., Chapochnikov, N.M., and Moser, T.: Spike encoding of neurotransmitter release timing by spiral ganglion neurons of the cochlea. J. Neurosci. 32 (14): 4773-4789, 2012. PMID: 22492033.

Safieddine, S. and Wenthold, R.J.: SNARE complex at the ribbon synapses of 
cochlear hair cells: analysis of synaptic vesicle- and synaptic membrane-associated proteins 11 (3): 803812, 1999.

Sakmann, B. and Neher, E.: Patch clamp techniques for studying ionic channels in excitable membranes. Annu. Rev. Physiol 46: 455-472, 1984. PMID: 6143532.

Schmitz, F., Knigstorfer, A., and Sdhof, T.C.: RIBEYE, a component of synaptic ribbons 28 (3): 857-872, 2000.

Schug, N., Braig, C., Zimmermann, U., Engel, J., Winter, H., Ruth, P., Blin, N., Pfister, M., Kalbacher, H., and Knipper, M.: Differential expression of otoferlin in brain, vestibular system, immature and mature cochlea of the rat 24 (12): 33723380, 2006.

Schuldiner, M., Metz, J., Schmid, V., Denic, V., Rakwalska, M., Schmitt, H.D., Schwappach, B., and Weissman, J.S.: The GET complex mediates insertion of tail-anchored proteins into the ER membrane. Cell 134 (4): 634-645, 2008. PMID: 18724936.

Schwander, M., Sczaniecka, A., Grillet, N., Bailey, J.S., Avenarius, M., Najmabadi, H., Steffy, B.M., Federe, G.C., Lagler, E.A., Banan, R., Hice, R., GrabowskiBoase, L., Keithley, E.M., Ryan, A.F., Housley, G.D., Wiltshire, T., Smith, R.J.H., Tarantino, L.M., and Mller, U.: A forward genetics screen in mice identifies recessive deafness traits and reveals that pejvakin is essential for outer hair cell function. J. Neurosci. 27 (9): 2163-2175, 2007.

Seal, R.P., Akil, O., Yi, E., Weber, C.M., Grant, L., Yoo, J., Clause, A., Kandler, K., Noebels, J.L., Glowatzki, E., Lustig, L.R., and Edwards, R.H.: Sensorineural deafness and seizures in mice lacking vesicular glutamate transporter 3. Neuron 57 (2): 263-275, 2008. PMID: 18215623 PMCID: PMC2293283.

Snellman, J., Mehta, B., Babai, N., Bartoletti, T.M., Akmentin, W., Francis, A., Matthews, G., Thoreson, W., and Zenisek, D.: Acute destruction of the synaptic ribbon reveals a role for the ribbon in vesicle priming. Nat Neurosci 14 (9): 1135-1141, 2011. PMID: 21785435. 
Sobkowicz, H.M. and Slapnick, S.M.: The efferents interconnecting auditory inner hair cells. Hear. Res. 75 (1-2): 81-92, 1994. PMID: 8071157.

Stefanovic, S. and Hegde, R.S.: Identification of a targeting factor for posttranslational membrane protein insertion into the ER 128 (6): 1147-1159, 2007.

Stefer, S., Reitz, S., Wang, F., Wild, K., Pang, Y.Y., Schwarz, D., Bomke, J., Hein, C., Lohr, F., Bernhard, F., Denic, V., Dotsch, V., and Sinning, I.: Structural basis for tail-anchored membrane protein biogenesis by the get3-receptor complex. Science 333 (6043): 758-762, 2011. PMID: 21719644 PMCID: PMC3601824.

Strenzke, N., Chanda, S., Kopp-Scheinpflug, C., Khimich, D., Reim, K., Bulankina, A.V., Neef, A., Wolf, F., Brose, N., Xu-Friedman, M.A., and Moser, T.: Complexin-i is required for high-fidelity transmission at the endbulb of held auditory synapse. J. Neurosci. 29 (25): 7991-8004, 2009.

Uthaiah, R.C. and Hudspeth, A.J.: Molecular anatomy of the hair cell's ribbon synapse. J. Neurosci. 30 (37): 12.387-12.399, 2010.

Vilardi, F., Lorenz, H., and Dobberstein, B.: WRB is the receptor for TRC40/Asna1-mediated insertion of tail-anchored proteins into the ER membrane. J. Cell. Sci. 124 (8): 1301-1307, 2011. PMID: 21444755.

Wang, F., Whynot, A., Tung, M., and Denic, V.: The mechanism of tail-anchored protein insertion into the ER membrane 43 (5): 738-750, 2011.

Wattenberg, B. and Lithgow, T.: Targeting of c-terminal (tail)-anchored proteins: Understanding how cytoplasmic activities are anchored to intracellular membranes 2 (1): 6671, 2001.

Winter, J., Jung, S., Keller, S., Gregory, R.I., and Diederichs, S.: Many roads to maturity: microRNA biogenesis pathways and their regulation. Nat Cell Biol 11 (3): 228-234, 2009.

Yamamoto, Y. and Sakisaka, T.: Molecular machinery for insertion of tail-anchored membrane proteins into the endoplasmic reticulum membrane in mammalian cells. Molecular Cell 48 (3): 387-397, 2012. 
Yasunaga, S., Grati, M., Cohen-Salmon, M., El-Amraoui, A., Mustapha, M., Salem, N., El-Zir, E., Loiselet, J., and Petit, C.: A mutation in OTOF, encoding otoferlin, a FER-1-like protein, causes DFNB9, a nonsyndromic form of deafness. Nat Genet 21 (4): 363-369, 1999.

Zhai, R.G. and Bellen, H.J.: The architecture of the active zone in the presynaptic nerve terminal. Physiology 19 (5): 262-270, 2004.

Zheng, J., Shen, W., He, D.Z., Long, K.B., Madison, L.D., and Dallos, P.: Prestin is the motor protein of cochlear outer hair cells 405 (6783): 149155, 2000. 
Contacts

EDUCATION

RESEARCH

EXPERIENCE

TEACHING

EXPERIENCE

\author{
Innear Ear Lab \\ Robert-Koch Strasse 40 \\ D-37075 Goettingen \\ Germany
}

\author{
E-mail: ilianapanou@gmail.com \\ Telephone: +49-(0)551-3922837 \\ Mobile: +49-(0)17693110207
}

Greek

Ph.D., Neuroscience

submitted

Georg-August University of Goettingen, Germany

Thesis title: "The role of tryptophan-rich basic protein (WRB) in inner hair cell synaptic transmission and hearing."

Diploma, Biology, grade 7.66/10

Aristotle University of Thessaloniki, Thessaloniki, Greece

Specialization in Molecular Biology, Genetics, and Biotechnology

High School Degree, Lyceum, grade 18.1/20

Geitonas Private High School, Athens, Greece

Apr 09 - Mar 13

Innear Ear Lab, Medical School, Goettingen, Germany

Advisors: Prof. Dr. Med. Tobias Moser, Dr. Tina Pangršič

Department of Neurology, Medical School, Goettingen, Germany

Jan 08 - Mar 09 Research Assistant

Project title: "Study of the mutant superoxide dismutase 1 in amyotrophic lateral sclerosis."

Advisor: Prof. Dr. Med. Mathias Baehr

Laboratory of Neurodegenerative Diseases, IIBEAA, Athens, Greece

Apr 07 - Nov 07

Research Intern

Project title: "Role of $\alpha$-synuclein in Parkinson disease."

Advisors: Dr. Leonidas Stefanis and Dr. Kostas Vekrellis

Center of Immunology and Transplantation, IIBEAA, Athens, Greece

Oct 06 - Nov 06 Research Trainee

Project title: "Study of the expression of the activin-like receptors (ALK) on HEK 293 and HeLa cell lines after infection with recombinant adenoviruses."

Advisor: Prof. Paschalis Sideras

Laboratory of Animal Physiology, AUTH, Thessaloniki, Greece

Oct 05 - Sep 06 Diploma Thesis

Thesis title: "Investigation of the expression of the peroxisome proliferator-activated receptors (PPAR) in the heart of diabetic and hypercholesterolemic rats and under conditions of ischemia and reperfusion." Advisor: Prof. Antigone Lazou

\section{Supervision of a master student}

Project title: "Silencing of otoferlin by miRNA - insights and applications".

May 11 - Jun 11

Supervision of an undergraduate student

Jul 11

Lab rotation

Training of a graduate student

Nov 12 - Feb 13 
AwARDS

SERvice

Method

Courses

TeChnical SKILlS

LANGUAGE SKILlS

Computer SKILLS

- Best talk award

- Appointed vice-speaker of the SMN program

$29^{\text {th }}$ Congress of Hellenic Society for Biological Sciences

May 2007

Expression of peroxisome proliferator activated receptors (PPAR) in diabetic and hypercholesterolemic rat heart. Effect of ischemia/reperfusion. (Poster Session)

Host : University of Athens and Aristotle University of Thessaloniki (AUTH).

Neuroprosthetics: from sensorimotor research to applications

Jun 2006

Symposium, Organizing Committee

Host: Students of the SMN program.

Biology of the Inner Ear: experimental and analytical approaches

Summer School MBL, Woods Hole, MA, USA

Mechanisms of RNA silencing

$13-14 / 10 / 2011$

Goettingen Graduate School for Neurosciences and Molecular Biosciences (GGNB)

ELECTRAIN, theoretical and hands-on course on Electrophysiology

Goettingen Graduate School for Neurosciences and Molecular Biosciences (GGNB)

Auditory and Visual Evoked Potentials

Goettingen Graduate School for Neurosciences and Molecular Biosciences (GGNB)

Time management in doctoral research

Goettingen Graduate School for Neurosciences and Molecular Biosciences (GGNB)

Advanced Light Microscopy

Goettingen Graduate School for Neurosciences and Molecular Biosciences (GGNB)

Advanced theoretical training course on fundamental principles in sensory processing

Max-Planck-Institute for Experimental Medicine, Goettingen.

Molecular biology

Cell cultures

Organotypic cultures (Organ of Corti)

Immuno-histo, -cytochemistry

Fluorescence microscopy, Confocal

Electrophysiology

Greek, native

English, fluent

German, fluent

Italian, conversational

WCIF Image, Igor Pro, Adobe Illustrator 